\title{
ADAPTIVE PLASTICITY AS A FITNESS BENEFIT OF MATE CHOICE
}

\author{
Patrick W. Kelly
}

\begin{abstract}
A dissertation submitted to the faculty at the University of North Carolina at Chapel Hill in partial fulfillment of the requirements for the degree of Doctor of Philosophy in the Department of Biology in the College of Arts and Sciences.
\end{abstract}

\section{Chapel Hill}

2021

Approved by:

David Pfennig

Karin Pfennig

Brian Langerhans

Christina Burch

Joel Kingsolver 
(C) 2021

Patrick W. Kelly

ALL RIGHTS RESERVED 


\begin{abstract}
Patrick W. Kelly: Adaptive Plasticity as a Fitness Benefit of Mate Choice (Under the direction of David Pfennig and Karin Pfennig)
\end{abstract}

Phenotypic plasticity and sexual selection can each promote adaptation in variable environments, but their combined influence on adaptive evolution is not well understood. In this dissertation, I propose that sexual selection can facilitate adaptation in variable environments when individuals prefer mates that produce adaptively plastic offspring. I develop this hypothesis and review existing studies showing that diverse groups display both sexual selection and plasticity in non-sexual traits. Thus, plasticity could be a widespread but unappreciated benefit of mate choice. I describe methods and opportunities to test this hypothesis and describe how sexual selection might foster the evolution of phenotypic plasticity. In addition, I detail two empirical examples of correlations between adult sexual traits and adaptive plasticity in offspring nonsexual traits. Understanding this interplay between sexual selection and phenotypic plasticity might help predict which species will adapt to a rapidly changing world. 
To my amazing wife, Julie, who never let me forget that I could accomplish a PhD, and to my parents, Dave and Sally, without whom none of my accomplishments would be possible. 


\section{ACKNOWLEDGEMENTS}

I am extremely grateful to my advisors, David Pfennig and Karin Pfennig, who gave me the opportunity to pursue a $\mathrm{PhD}$, taught me how to do research, brought me into the spadefoot toad fold, and showed me just how fun a field season can be. In addition, I am very

thankful for the support, education, instruction, advice, and patience afforded me by my all of my committee members, who include the Pfennigs, Christina Burch, Brian Langerhans, and Joel Kingsolver.

My family, including of course, my parents, Sally and Dave, my brother, Mike, and his wife, Tana, my cousin (sister), Mary, and her beautiful family, and many others gave me immense support and encouragement throughout my tenure as a graduate student. I will forever be grateful to all of them.

The same is true of my friends and lab mates, one and the same, who became some of the best friends I've ever had. And while each of them is special to me, I would like to thank Gina Calabrese and her partner, Jacob Heiling, in particular. You became my North Carolina family. I hope that we never live far from each other.

Finally, I can never thank my wife, Julie, enough for everything she has done to keep me on track, support me in every way, and be the best friend that I needed all along. 


\section{TABLE OF CONTENTS}

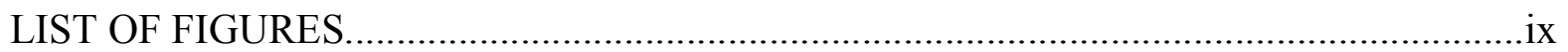

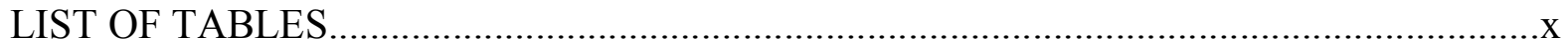

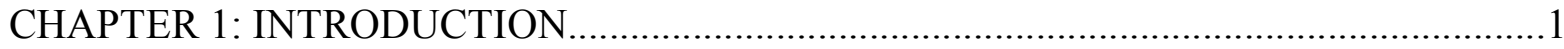

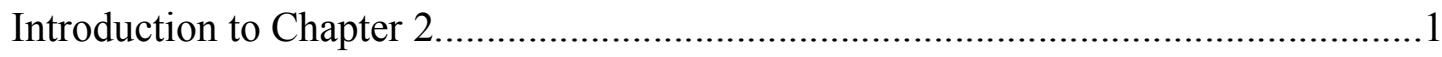

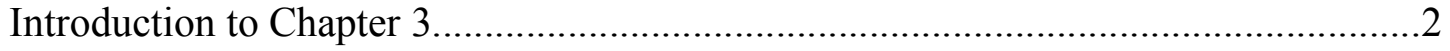

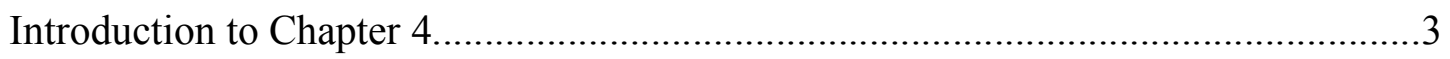

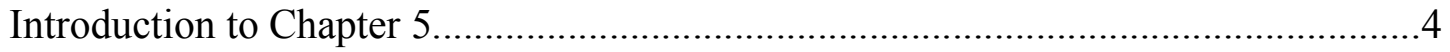

CHAPTER 2: ADAPTIVE PLASTICITY AS A FITNESS

BENEFIT OF MATE CHOICE.....................................................................................

Phenotypic Plasticity and Sexual Selection..........................................................

Offspring Plasticity as a Fitness Benefit of Mate Choice........................................ 7

Sexual Signals as Indicators of Offspring Plasticity......................................... 10

Inheritance of Plasticity: Genetic and Nongenetic Mechanisms.............................14

Evolutionary Implications of Offspring Plasticity as a

Fitness Benefit of Mate Choice............................................................................... 17

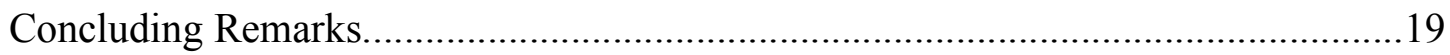

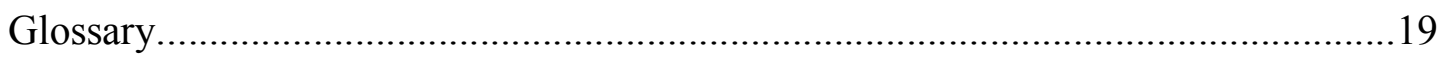

Box 1: A Context Where Offspring Plasticity is Likely to

Serve as a Fitness Benefit of Mate Choice..........................................................21

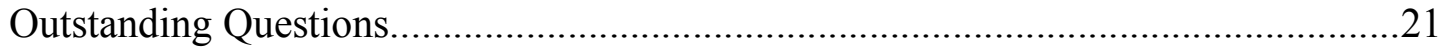




\section{CHAPTER 3: MALE SEXUAL SIGNAL PREDICTS PLASTICITY \\ IN OFFSPRING: IMPLICATIONS FOR THE EVOLUTION OF}

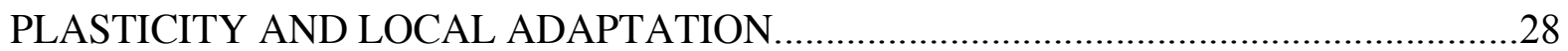

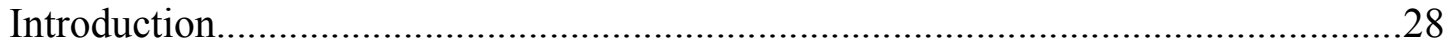

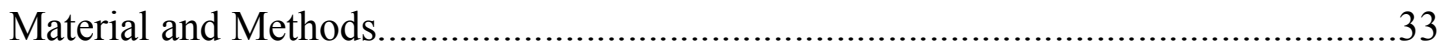

(a) Study System and Specific Goals........................................................33

(b) Does a Male Sexual Signal Predict Production of an

Environmentally-Induced Phenotype in His Offspring?

(c) Does a Male Sexual Signal Predict Adaptive

Offspring Plasticity?

Results.

(a) Does a Male Sexual Signal Predict Offspring

Resource-use Morphology?

(b) Does a Male Sexual Signal Predict Offspring Plasticity?

Discussion.

CHAPTER 4: A CONDITION-DEPENDENT MALE SEXUAL

SIGNAL PREDICTS ADAPTIVE PREDATOR-INDUCED

PLASTICITY IN OFFSPRING

Introduction.

Material and Methods.

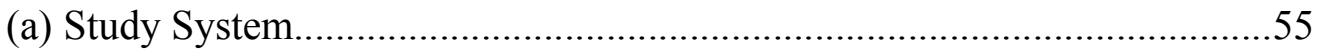

(b) Field Collections and Tadpole Rearing..............................................56

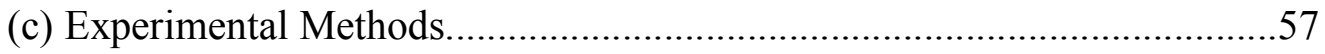

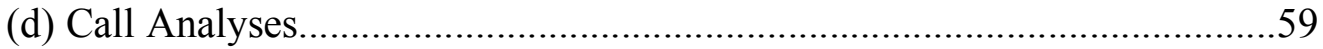

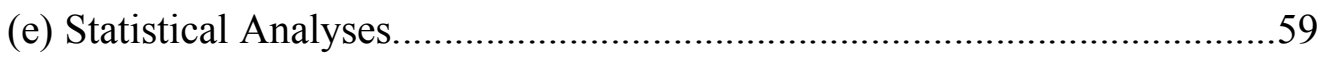

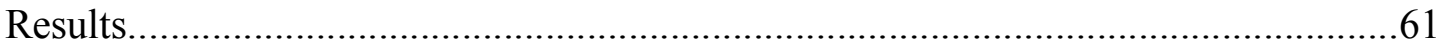

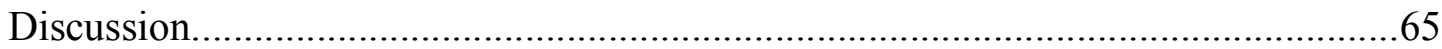


What are the genetic, developmental, and evolutionary mechanisms by which offspring plasticity becomes associated with adult sexual traits?.

Are there particular traits, taxa or conditions in which sexual traits are more likely to predict offspring plasticity?

Can mate choice for offspring plasticity explain some of the conflicting evidence for indirect benefits of mate choice? 61

Can sexual selection, paradoxically, lead to the loss of plasticity?............................74

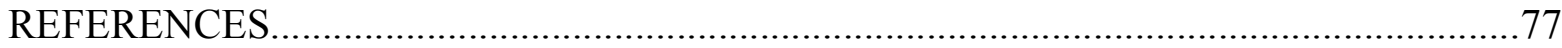




\section{LIST OF FIGURES}

\section{CHAPTER 1}

Figure 1 - An Example in Which Offspring Plasticity Serves as a

Fitness Benefit of Mate Choice.... 8

Figure 2 - A Method for Evaluating if Offspring Plasticity is a

Fitness Benefit of Mate Choice

\section{CHAPTER 2}

Figure 1 - Hypothetical Examples of Potential Effects of Environmental Variability on Whether Mate Choice Might Impede or Facilitate Local Adaptation.

Figure 2 - The Relationship Between Sire Call Rate and The Number of Tadpoles That Developed Carnivore Morphology

Figure 3 - The Relationship Between Sire Call Rate and Plasticity

in Tadpole Resource-Use Morphology....

Figure 4 - Reaction Norms of Family Mean Morphological

Index Values When Tadpoles Were Reared on Detritus

Versus Shrimp Diets.

\section{CHAPTER 3}

Figure 1 - Mean SVL-Corrected Tail Depth With 95\%

Bootstrapped CIs for All Tadpoles Reared in the Control and

Cue Treatments.

Figure 2-SVL-Corrected Tail Depths (Points) and

Box-And-Whisker Plots for Each Sibship and Each Treatment

Figure 3 - Linear Regressions of Tadpole Plasticity on Sire

Call Effort and Sire Body Condition...... .64 


\section{LIST OF TABLES}

\section{CHAPTER 1}

Table 1 - General Conditions Favoring the Evolution of Mate

Preferences for Individuals That Produce Adaptively Plastic Offspring...................23

Table 2 - Evidence of Genetic Variation in Plasticity as Well as of

Sexual Selection in Selected Animals.....................................................................24

\section{CHAPTER 2}

Table 1 - Alternative Routes Whereby Sexual Selection Can

Impact the Evolution of Phenotypic Plasticity 


\section{CHAPTER 1: INTRODUCTION}

Here, I provide brief summaries of each of the later chapters. I intend that this chapter and its summaries of the others serve as a guide to this dissertation and to the ways in which the chapters relate to each other and together form a singular body of theory and data on adaptive plasticity as a fitness benefit of mate choice and the ecological and evolutionary implications thereof.

These summaries are adapted from the published abstracts of the peer-reviewed versions of the chapters to which they correspond. I have included citations to those publications here and in each of the corresponding chapters.

\section{Introduction to Chapter 2: Adaptive Plasticity as a Fitness Benefit of Mate Choice}

The following is adapted from:

Kelly, P. W., Pfennig, D. W., \& Pfennig, K. S. 2021. Adaptive Plasticity as a Fitness Benefit of Mate Choice. Trends in Ecology \& Evolution. 36: 4.

Phenotypic plasticity and sexual selection can each promote adaptation in variable environments, but their combined influence on adaptive evolution is not well understood. In this dissertation, I propose that sexual selection can facilitate adaptation in variable environments when individuals prefer mates that produce adaptively plastic offspring. In Chapter 2, I develop this hypothesis and review existing studies showing that diverse groups display both sexual selection and plasticity in non-sexual traits. Thus, plasticity could be a widespread but unappreciated benefit of mate choice. I additionally describe in Chapter 2 
methods and opportunities to test this hypothesis and describe how sexual selection might foster the evolution of phenotypic plasticity. Understanding this interplay between sexual selection and phenotypic plasticity might help predict which species will adapt to a rapidly changing world.

\section{Introduction to Chapter 3: Male Sexual Signal Predicts Phenotypic Plasticity in Offspring: Implications for the Evolution of Plasticity and Local Adaptation}

The following is adapted from:

Kelly, P.W., Pfennig, D.W., de la Serna, S., and Pfennig, K.S. 2019. Male sexual signal predicts phenotypic plasticity in offspring: implications for the evolution of plasticity and local adaptation. Philosophical Transactions of the Royal Society B. 374: 20180179.

In a rapidly changing world, understanding the processes that influence a population's ability to respond to natural selection is critical for identifying how to preserve biodiversity. Two such processes are phenotypic plasticity and sexual selection. Whereas plasticity can facilitate local adaptation, sexual selection potentially impedes local adaptation, especially in rapidly changing or variable environments. In Chapter 3, I hypothesize that, when females preferentially choose males that sire plastic offspring, sexual selection can actually facilitate local adaptation to variable or novel environments by promoting the evolution of adaptive plasticity. I tested this hypothesis by evaluating whether male sexual signals could indicate plasticity in their offspring and, concomitantly, their offspring's ability to produce locally adapted phenotypes. Using spadefoot toads (Spea multiplicata) as my experimental system, I show that a male sexual signal predicts plasticity in his offspring's resource-use morphology. Specifically, faster-calling males (which are preferred by females) produce more plastic 
offspring; such plasticity, in turn, enables these males' offspring to respond adaptively to the spadefoots' highly variable environment. This association between a preferred male signal and adaptive plasticity in his offspring suggests that female mate choice can favor the evolution and maintenance of phenotypic plasticity and thereby foster adaptation to a variable environment.

\section{Introduction to Chapter 4: A Condition-Dependent Male Sexual Signal Predicts Adaptive Predator-Induced Plasticity in Offspring}

The following is adapted from:

Kelly, P.W., Pfennig D.W., and Pfennig, K.S. 2021. A condition-dependent male sexual signal predicts adaptive predator-induced plasticity in offspring. Behavioral Ecology \& Sociobiology. 75: 28.

The possibility that sexual selection promotes adaptive evolution in variable environments remains controversial. In particular, where the scale of environmental variation results in parents and their offspring experiencing different environmental conditions, such variation is expected to break down associations between adult sexual traits and adaptive offspring traits. However, when adaptive offspring plasticity in nonsexual traits acts as an indirect benefit of mate choice, then mate choice for males that produce more plastic offspring could promote adaptation to variable environments. This hypothesis assumes that male sexual signals predict offspring plasticity, which has rarely been tested.

In Chapter 4, I used spadefoot toads (Spea multiplicata) to investigate whether variation in male sexual signals predicts the expression of tadpole tail-fin plasticity in response to predation cues. Such plasticity has been shown to be adaptive in numerous amphibian taxa. I found that condition-dependent male call characteristics predicted 
offspring plasticity. Generally, both phenotypic plasticity and female mate choice are ubiquitous in nature; therefore, adaptive associations between male sexual signals and offspring plasticity such as the one reported here might be common.

\section{Introduction to Chapter 5: Concluding Remarks}

In Chapter 5, I return to the section in Chapter 2 called "Outstanding Questions," which described a series of challenges to help guide future research into the evolutionary and ecological importance of adaptive plasticity as a fitness benefit of mate choice. I turned each of these questions into a subheading and gave my current theoretical understanding of, and predictions for, the challenges that they pose, along with more-detailed suggestions for future empirical inquiry. I hope that Chapter 5, along with this dissertation as a whole, will inspire a great deal of important future work in this emerging field. [5] 


\section{CHAPTER 2: ADAPTIVE PLASTICITY AS A FITNESS BENEFIT OF MATE CHOICE}

The following is adapted from:

Kelly, P. W., Pfennig, D. W., \& Pfennig, K. S. 2021. Adaptive Plasticity as a Fitness Benefit of Mate Choice. Trends in Ecology \& Evolution. 36: 4.

\section{Phenotypic Plasticity and Sexual Selection}

Researchers have long recognized that sexual selection and phenotypic plasticity (see Glossary) can each play key roles in diverse ecological and evolutionary processes [69]. Less widely appreciated is that sexual selection and plasticity can interact in ways that influence how these processes unfold. One such process that this interaction can impact is adaptive evolution.

A way that sexual selection-acting alone_-can promote adaptive evolution is when sexual traits (e.g., female mate preferences or male sexual signals) predict offspring fitness [10-13]. For example, males might produce condition-dependent sexual displays that predict the fitness of their offspring (e.g., because good-condition males transmit fewer deleterious and more beneficial alleles to their offspring relative to lower-condition males [10-15]). If females preferentially mate with males that express exaggerated forms of those conditiondependent displays — and produce fitter offspring as a result — sexual selection thereby fosters adaptation [12-15]. However, some have argued that maintaining this type of relationship between sexual traits and offspring fitness requires consistent selection [16-18]. This is 
problematic, because most environments vary in space and time. Such variation can cause the selective environments that adults experience to differ from those that their offspring experience. In these situations, sexual traits would likely fail to predict offspring fitness, which would preclude sexual selection from promoting adaptation [8, 18-21].

Sexual selection could promote adaptation in variable environments by favoring plasticity in mate preferences or sexual signals $[8,11,17,21,22]$. Under such circumstances, sexual traits that maximize offspring fitness in a particular environmental context would be expressed only in that context when parents assess the environment and respond by altering their sexual traits though plasticity. Yet, if parental and offspring environments are so different that parents cannot assess the conditions that their offspring will experience, then even such plasticity in sexual traits would fail to promote adaptive evolution [4, 16-21]. Moreover, offspring of a given pairing might experience selective pressures that differ not only from those experienced by the parents, but also from those experienced by their siblings. Thus, plasticity in parents' sexual traits might be unable to optimize the fitness of all their offspring $[8,18,23]$.

Yet, there is a way that sexual selection can promote adaptation, even in variable environments where parents cannot assess their offspring's environment(s) or in situations where parents cannot facultatively modify their sexual traits. Specifically, individuals could choose mates that produce adaptively plastic offspring. Essentially, individuals could select mates whose offspring adaptively assess and respond to their environment [4]; in other words, offspring plasticity could be a fitness benefit of mate choice. Such offspring plasticity would arise when individuals choose mates that either pass on genetic or non-genetic variation to offspring that enables offspring to respond facultatively to the environment, or 
provide resources to the choosy individual that enables the production of plastic offspring (e.g., nuptial gifts [sensu 24] that, when allocated to offspring, enable greater plasticity). Regardless of how the benefit of offspring plasticity is conferred, plasticity in offspring traits—as opposed to plasticity in adult sexual traits—should arise under conditions where parent and offspring environments differ and where parental plasticity is either not possible or disfavored (Table 1; see also Box 1).

The hypothesis that offspring plasticity could function as a fitness benefit of mate choice rests on conventional predictions of mate choice theory [sensu 6]. Nevertheless, the potential for plasticity and sexual selection to interact in this way has important implications for both how adaptation can proceed in variable environments and the evolution of plasticity per se. Before discussing these evolutionary implications, we first develop the hypothesis in greater detail.

\section{Offspring Plasticity as a Fitness Benefit of Mate Choice}

When individuals prefer mates that produce plastic offspring, such offspring plasticity could constitute a fitness benefit of mate choice. Offspring plasticity could take many forms (Table 2), including: the expression of alternative morphologies in early development [4, 25], greater behavioral flexibility in coping with their environment [26], or even the expression of adult traits $[8,11,17,21,22]$. However, our focus here is plasticity involving offspring (not parents) assessing and responding to their environment and that is expressed prior to sexual maturity (Table 1). Such plasticity is common in nature and frequently influences offspring survival (Table 2). Importantly, plasticity expressed prior to maturity is not only a potential 
fitness benefit of mate choice, but can also continue to be expressed and/or have important

effects on phenotypes and fitness in sexually mature individuals (see Table 2).

To illustrate how plasticity can serve as a fitness benefit of mate choice, consider

Mexican spadefoot toads (Spea multiplicata; Figure 1). Spea tadpoles can develop as either`

(A)

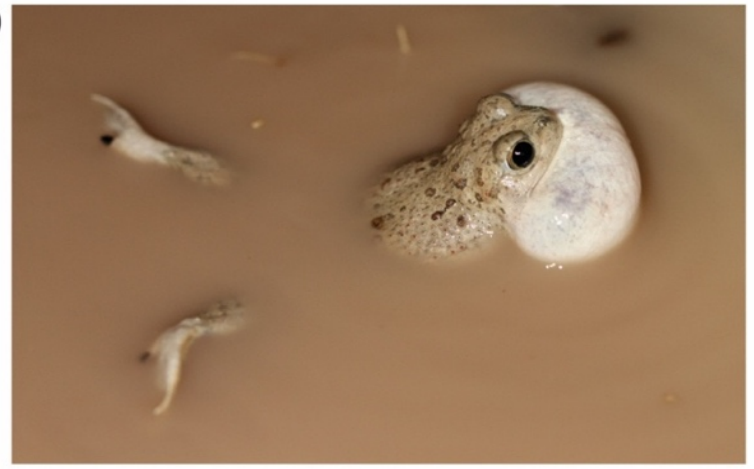

(C)

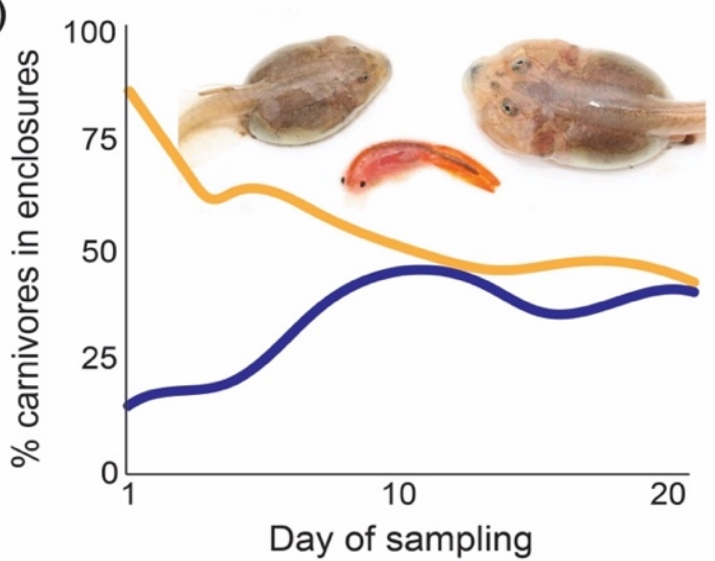

(B)

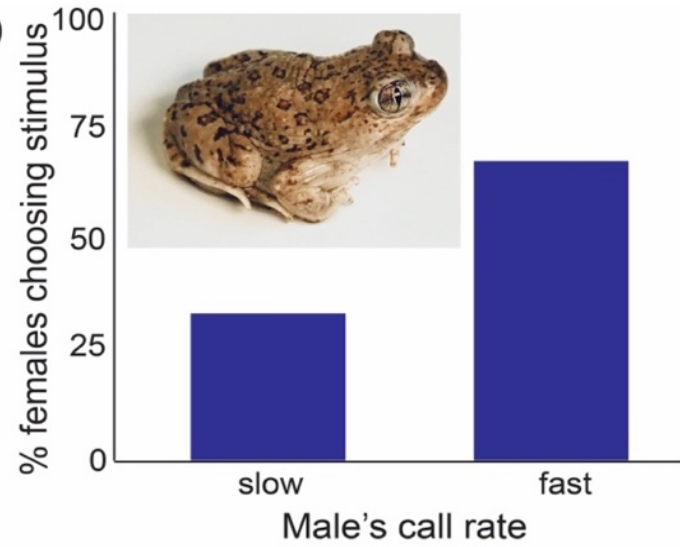

(D)

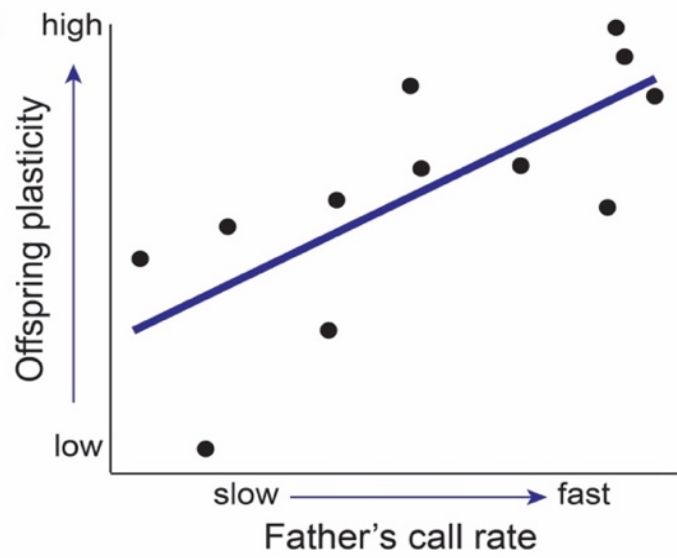

Figure 1. An Example in which Offspring Plasticity Serves as Benefit of Mate Choice. (A) Male Mexican spadefoot toads, Spea multiplicata, call to attract females as mates, and (B) females prefer males that call faster. (C) Their tadpoles have evolved pronounced diet-induced plasticity, developing into either an omnivore morph (left photo) or a carnivore morph (right photo), which is induced by, and specializes on, Anostracan fairy shrimp (middle photo). This plasticity is favored by frequency dependent natural selection, such that the optimal phenotype for an individual to produce depends, in part, on what others do. As evidence of such selection, when the frequency of carnivores in different enclosed areas of a natural pond is either increased (blue line in graph) or decreased (orange line), this frequency of carnivores later converges on a common, equilibrium frequency, which varies from pond to pond, depending on the relative abundance of shrimp. (D) This also means that parents cannot predict the optimal tadpole phenotype; instead, selection favors adaptively plastic offspring because offspring are in a better position to evaluate their selective environment (i.e., the resource base and number of competitors). Consistent with this expectation, males that display the signal preferred by females -i.e., faster-calling males - sire offspring that are more likely to express carnivore-omnivore plasticity. References for data in figures: (B) - [2]; (C) - [3]; (D) - [4]. 
an omnivore phenotype with generalized tadpole morphology and diet, or as a distinctive carnivore phenotype, which possesses features that allow it to specialize on large, mobile animal prey, such as Anostracan fairy shrimp or other tadpoles [3]. This carnivore phenotype is induced when a young tadpole eats fairy shrimp or other tadpoles [27]; i.e., Spea tadpoles exhibit diet-induced phenotypic plasticity.

Neither alternative phenotype is intrinsically superior to the other. Rather, intraspecific resource competition gives rise to negative frequency-dependent disruptive selection, which maintains both phenotypes in the same pond [3]. Such selection generates equilibrium frequencies of the two phenotypes, which vary across ponds and years [3]. Consequently, the optimal developmental strategy for a tadpole is to possess plasticity to become either an omnivore or a carnivore [3,27]. Critically, this plasticity is greatest among S. multiplicata tadpoles sired by the relatively high-condition, fast-calling males with which females prefer to mate $[2,4,28,29]$.

The Spea example underscores the key components of the hypothesis that offspring plasticity can serve as a fitness benefit of mate choice: sexual signals can predict the extent to which a prospective mate's offspring are plastic; individuals prefer mates who possess traits indicative of their ability to produce more plastic offspring; and, because tadpole fitness is frequency dependent, the offspring — not the parents - are best able to assess prevailing environmental conditions and express adaptive plastic responses to them (Figure 1; see also Table 1).

Offspring plasticity might serve as a benefit of mate choice in many systems, given that both sexual selection and genetic variation in plasticity has been reported in numerous studies (Table 2). In particular, variation in the slopes, heights, and shapes of reaction norms 
among sibships (genotypes) suggests that heritable (e.g., genetic) variation among reaction norms could underlie a relationship between adult sexual traits and offspring plasticity. Importantly, in a number of the systems where sibship-level variation in reaction norms is observed, sexual selection has also been documented (Table 2). Although the details of male sexual signals and female mate choice are not always known, this broad distribution of both plasticity and sexual selection point to the general potential for plasticity to serve as a fitness benefit of mate choice.

\section{Sexual Signals as Indicators of Offspring Plasticity}

For offspring plasticity to serve as a fitness benefit of mate choice requires that individuals gauge a prospective mate's ability to produce plastic offspring. Direct assessment of a potential mate's ability to do so is unlikely $[6,8]$, especially in contexts where offspring plasticity is most likely favored (Table 1; Box 1). Instead, individuals should rely on sexual signals that predict a prospective mate's capacity to produce plastic offspring $[6,8,30,31]$.

Generally, for sexual traits to predict offspring fitness, these traits must be conditiondependent or otherwise costly to produce $[6,8,10,23,31,32]$; i.e., they must be "honest" signals [31]. Thus, in the case of offspring plasticity, only those individuals capable of allocating resources to signaling can express preferred sexual signals, and these signals would reliably predict an individual's ability to produce plastic offspring. Moreover, those individuals' offspring will inherit the capacity to produce the preferred sexual signals as a reflection of their fitness. If offspring plasticity is selectively favored in variable environments, then those offspring capable of facultatively expressing adaptive phenotypes in a given environment would attain the condition necessary to produce those signals as 
adults. Consequently, offspring plasticity might readily couple offspring fitness with the ability to express preferred signals [sensu 33, 34, 35].

For sexual signals and offspring plasticity to become correlated does not require that mate preferences favor offspring plasticity per se, nor does it hinge on specific assumptions regarding the evolutionary origins of mate preferences. Indeed, if there is no variation in mate preferences (e.g., because the preference is fixed in the population or because individuals mate randomly with respect to sexual signals), then individuals that choose mates that produce more plastic offspring will benefit from those matings. Similarly, individuals might prefer mates that produce more energetic signals, either because such signals are more stimulatory to the nervous system or because they correspond to other fitness benefits (e.g., fertilization success, parental care or nuptial gifts). However, if such mates also produce adaptively plastic offspring, then individuals will receive this benefit regardless of the origins of the preference.

Moreover, the possibility that offspring plasticity serves as a fitness benefit of mate choice addresses one of the general arguments that environmental variation disrupts predictive relationships between sexual traits and offspring fitness. In particular, some have argued that environmental variation — and even the expression of phenotypic plasticity in response to that variation-should weaken correlations between genotypes and phenotypes, thereby diminishing the likelihood that sexual signals predict offspring fitness [8, 16, 18-20]. Yet, such arguments likely only apply when the environment varies unpredictably [see, e.g., 18]. By contrast, adaptive plasticity is not expected to evolve in unpredictable environments [36-40]. Instead, adaptive plasticity is expected to evolve in variable environments where salient cues reliably predict prevailing or impending environmental conditions [39, 41]. For 
example, as highlighted above, reaction norms evolve in response to selection favoring individuals that express the appropriate phenotype in a given environment [7, 42-44]. Thus, selection can refine both the extent to which offspring are plastic and the phenotypes that are expressed in different environments, given those environments are of the sort that are expected to favor plasticity in general $[7,39,45,46]$. In these situations, plasticity does not preclude a predictive relationship between sexual traits and offspring fitness, but could instead reinforce such a relationship.

Establishing that plasticity serves as a fitness benefit of mate choice requires evaluating whether preferred sexual traits predict offspring plasticity and fitness (Figure 2; e.g., [4]). In performing such tests, it is critical to measure offspring fitness across different environments (Figure 2). Indeed, in any given environment, offspring with fixed phenotypes that are optimal for that environment might achieve higher fitness than offspring with facultatively expressed phenotypes [sensu 47, 48]. Yet, those same offspring with that fixed phenotype would do poorly in an alternative environment compared to offspring that can facultatively express traits that are better suited to the alternative environment. Thus, offspring that express different phenotypes in response to the environment should have higher fitness overall when averaged across multiple environments [9].

In this regard, offspring plasticity differs from other fitness benefits ascribed to mate choice. If plastic individuals are unable to express the optimum phenotype in a given environment, then the offspring of preferred mates or those with exaggerated sexual signals might not be those with the highest fitness in that environment. The possibility that offspring plasticity could serve as a benefit in this way could explain why preferred males with exaggerated sexual signals do not always sire the fittest offspring in any particular 
environment [8]. Thus, identifying whether offspring plasticity constitutes a fitness benefit of mate choice could reconcile, at least in part, longstanding issues about the nature and prevalence of indirect, "good genes" benefits of mate choice [8,23].
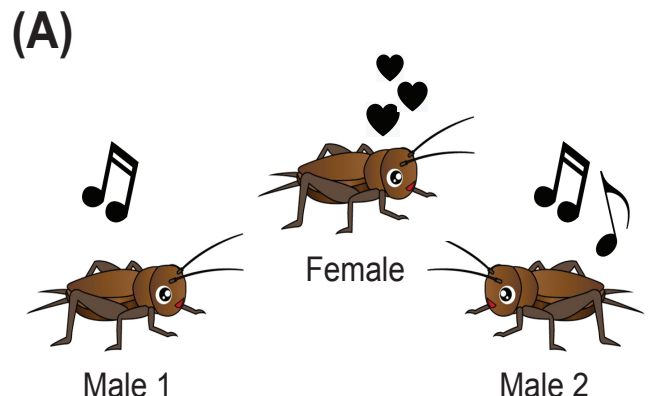

Male 1
(B)

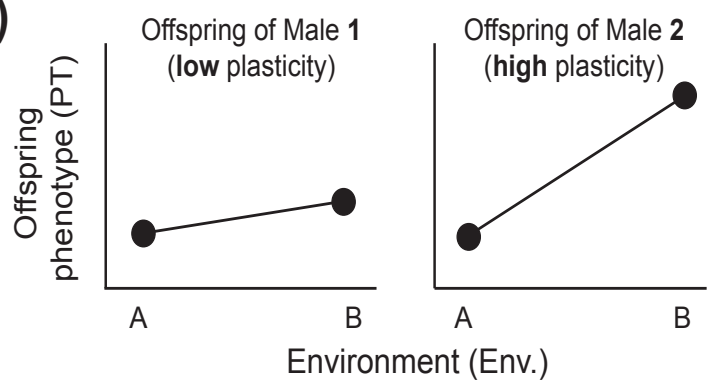

Estimate for each male his offsprings' plasticity (= the mean offspring PT in Env. B - A)

(D)

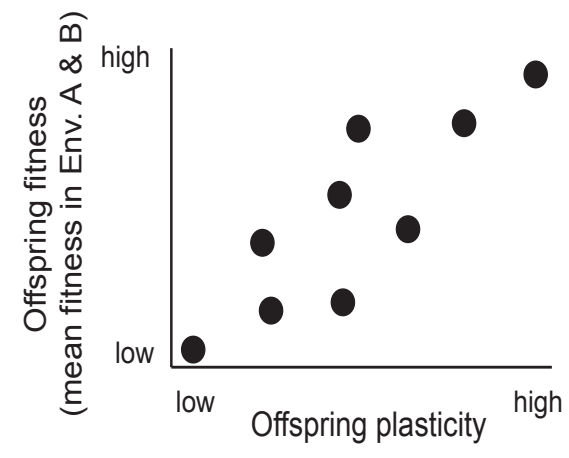

Assess relationship between offspring plasticity and offspring fitness (averaged across environments)

Figure 2. A Method for Evaluating if Offspring Plasticity is a Fitness Benefit of Mate Choice. (A) First, determine if certain male signals are preferred by females. (B) Second, use a common-garden approach to determine if offspring of different males vary in degree of ecologically relevant plasticity (note that in doing so one should incorporate methods that account for possible parental effects, such as differential maternal resource allocation, impacting offspring plasticity [19]). From this, ask whether the slopes of the reaction norms are steeper for preferred than for non-preferred males or, alternatively, (C) examine the relationship between the male signal and offspring plasticity, where the latter can be estimated by taking the family mean difference in phenotype when offspring are exposed to two (or more) environments (e.g., obtained from a reaction norm experiment as shown in panel B). (D) Finally, determine if the plasticity is adaptive by measuring the fitness of the offspring from different males when they are reared in the different environments that they would normally encounter in nature; more plastic offspring should have higher fitness than less plastic offspring across these different environments. Note that more plastic offspring might not have the highest fitness in a given environment (see text), but they should have higher average fitness across environments. 


\section{Inheritance of Plasticity: Genetic and Non-Genetic Mechanisms}

A predictive association between sexual signals and offspring plasticity does not require a genetic basis to plasticity in offspring (or to sexual traits) but such a genetic basis may exist. Indeed, as highlighted in Table 2, numerous studies have documented variation among sibships and populations in whether and how they respond to any particular change in their environment. Moreover, plastic traits commonly exhibit additive genetic variance in the heights and slopes of reaction norms $[42,44,49,50]$. Both observations suggest the presence of underlying heritable variation in plasticity.

Attempts to identify specific genes that regulate plasticity—and that could serve as the targets of sexual selection for offspring plasticity—are still in their infancy [51], but such attempts have produced some intriguing possibilities. For instance, researchers have identified several developmental switch genes that influence plasticity [e.g., 52, 53, 54]. In situations where, as a result of mate choice, offspring inherit alleles at specific genes that render them more plastic, plasticity could constitute an indirect, "good genes" benefit of mate choice.

However, the hypothesis that offspring plasticity can serve as a fitness benefit of mate choice does not necessitate that offspring actually inherit alleles for plasticity. Rather, offspring may inherit non-genetic factors [sensu 55]. Indeed, increasing evidence suggests that such non-genetic factors as epigenetic modifications of DNA play a key role in mediating plasticity $[55,56]$. For instance, some of the aforementioned developmental switch genes that regulate plasticity are themselves influenced by epigenetic factors $[53,54]$. This suggests that mechanisms of non-genetic inheritance and the genetic architecture of plasticity 
can both be targets of selection and together shape the evolutionary trajectories of plastic traits in offspring.

In short, the mechanisms mediating the inheritance and expression of plasticity—and that could therefore serve as targets of selection for offspring plasticity-are varied and complex $[9,51]$. Indeed, both genetic and non-genetic mechanisms might play important roles in the expression of offspring plasticity, and these mechanisms will differentially impact whether and how sexual selection is exerted on the genome and the subsequent evolutionary response. Thus, a key challenge is to uncover the factor(s) actually transmitted across generations that influences offspring plasticity (see Outstanding Questions).

Regardless of whether sexual signals become correlated with genetic or inherited non-genetic (e.g., epigenetic) variation underlying plasticity [10, 12], offspring plasticity would still constitute a fitness benefit of mate choice.

An important consideration regarding offspring plasticity as a benefit of mate choice is the subsequent impact of directional selection exerted by mate choice on the underlying genetic or non-genetic variation. If mate choice exerts strong selection on sexual signals that are indicative of adaptive, facultatively expressed traits, then any underlying heritable variation for those signals or the facultatively expressed traits could be lost over time [6]. Historically, variable selection (and, therefore, shifting optima) was one explanation for how variation could be maintained in the face of strong selection $[6,16,19]$. Yet, under the scenario we outline here, the same genetic variants would be favored regardless of environment (because the plastic variants exhibit higher fitness when averaged across all environments). Thus, genetic variation could decline as populations approached optima for sexual signals and offspring plasticity. 
However, the genetic (and/or non-genetic) architecture of plasticity likely consists of a sufficiently large mutational target to maintain heritable variation in the face of persistent selection by mate preferences (analogous to "genic capture" [sensu 10, 32]). Indeed, the genetic architecture of adaptive plasticity — and of the resulting facultatively induced phenotypes-likely consists of numerous loci with diverse functions, ranging from elements of the sensory system involved in detection of environmental cues to the threshold amount of hormone needed to trigger a specific plastic response $[9,51]$. Such diversity would provide ample potential sources of heritable variation across the genome that is unlikely to be exhausted by sexual selection, especially if multiple routes exist by which facultative expression of adaptive phenotypes exist [57].

Generally, the complexity of mechanisms underlying adaptive plasticity is thought to explain the maintenance of genetic variation in plasticity in natural populations $[50,58]$, and it suggests that the potential for a single, optimally plastic genotype to approach fixation is low. Whether this is the case remains to be tested, but we suspect that the variation underlying offspring plasticity and sexual traits might be especially resistant to depletion. Moreover, if condition-dependent direct benefits, such as the provisioning of nuptial gifts and subsequent maternal resource allocation to offspring, also contribute to mate choice favoring offspring plasticity, then heritable variation should readily be maintained $[8,24]$. As greater attention is given to offspring plasticity as a benefit of mate choice, theoretical and empirical work should evaluate how variation in phenotypic plasticity (whether caused by genetic or non-genetic mechanisms) is impacted by mate choice and the subsequent effect of this variation on sexual selection. 


\section{Evolutionary Implications of Offspring Plasticity as a Fitness Benefit of Mate Choice}

That offspring plasticity can function as a benefit of mate choice has at least two key evolutionary implications. First, it can help explain how sexual selection promotes adaptation in novel or variable environments. As noted above, a criticism of the notion that sexual selection promotes adaptation is that environmental variation will break down any correlations between adult sexual traits and offspring fitness. However, if sexual traits indicate the capacity for offspring to produce different traits in response to prevailing or impending environmental conditions, then adult sexual traits will predict offspring fitness even in variable environments [4] (Figure 2).

Moreover, exposure to novel environments can induce the expression of plasticity, which is then subject to novel selective pressures $[59,60]$. This plasticity can be adaptive in the sense that it allows rapid (within-generation) responses to selection and subsequent shortterm population persistence, but whether it allows long-term persistence depends on the existence of underlying heritable variation in plasticity and, thus, the capacity for evolutionary responses to selection $[50,58,60]$. If inducing cues in a novel environment are reliably predictive of environmental conditions [sensu 61], then the potential for a long-term adaptive response may be increased by associations between sexual traits and offspring plasticity owing to the combined effects of direct ecological selection and indirect sexual selection. Indeed, because selection on sexual traits can be stronger than selection on viability and fecundity traits in natural populations $[62,63]$, offspring plasticity as a benefit of mate choice might facilitate relatively rapid adaptive evolution and long-term persistence in novel environments. Testing this possibility is especially important in a time of global change. 
A second implication of offspring plasticity as a benefit of mate choice is that it could impact the evolution of phenotypic plasticity itself [4]. Plasticity is increasingly thought to play diverse roles in ecology and evolution, including enabling populations to persist in novel or changing environments and facilitating speciation $[7,59,60,64-66]$. Plasticity can assume such diverse roles because plasticity can itself evolve [67], and its evolution can, in turn, have important downstream consequences $[45,68]$. For example, because environmental change is typically harmful to an organism (it reduces the match between its phenotype and environment), phenotypic plasticity has evolved in essentially all taxa [69], where it allows individuals to produce phenotypes that are better suited for any new conditions that they might encounter $[7,9]$. However, because different genotypes typically vary in whether and how they respond to any given environmental change (Table 2), selection can act on this formerly cryptic genetic variation for plasticity [70] and disfavor those genotypes that produce poorly adapted phenotypes for the current environment. Through this process of adaptive refinement (known as "genetic accommodation" [7]), selection can cause a change in both the regulation of plasticity and form of the facultatively expressed phenotypes, leading to a better match between phenotype and environment $[7,59,65,66]$.

Sexual selection might play a crucial role in this process of plasticity-led evolution. If mate choice enhances the reproductive success of individuals that produce plastic offspring, then mate choice would thereby increase the production of offspring capable of responding to cues that reliably indicate prevailing or impending environmental conditions. As more plastic offspring are produced and exposed to selection, the opportunity for selection to refine that plasticity is increased $[4,7,45]$. Thus, when adult sexual traits 
become associated with adaptive offspring plasticity, they may readily enable plasticity-led evolution to facilitate adaptation $[4,7,45,66,68]$.

\section{Concluding Remarks}

The potential for offspring plasticity to constitute a fitness benefit of mate choice could arise whenever the environment is spatially or temporally variable and offspring are better able than their parents to accurately assess their environment or express plasticity (Table 1). Evaluating whether plasticity can serve as a fitness benefit of mate choice could explain patterns of sexual selection in diverse taxa and help resolve whether and how sexual selection contributes to adaptation, which is an enduring issue in evolution $[6,15,17,71-74]$. Moreover, because mate choice for plastic offspring could facilitate the evolution of plasticity, sexual selection could amplify plasticity-led evolution and its downstream consequences. Given that many species are experiencing rapid environmental change, and that plasticity might help populations "buy time" until more permanent adaptive solutions can evolve [60], evaluating whether plasticity can serve as a benefit of mate choice is especially timely now (see Outstanding Questions).

\section{Glossary}

Cryptic genetic variation: Genetic variation that is not expressed phenotypically under normal conditions. Such variation can be revealed phenotypically when a population experiences a new environment; i.e., it can be revealed through plasticity. 
Mate preference: Any sensory, behavioral, or other trait of the choosing sex that shapes, in part, individuals' likelihoods of mating with members of the opposite sex that express certain phenotypes.

Non-genetic factors: Any one of the various epigenetic factors (e.g., patterns of DNA methylation), behavioral factors (e.g., cultural transmission), or environmental factors (e.g., noncoding RNAs or micro-organisms) that, independently of DNA sequence variation, can influence phenotype production, be transmitted across generations, and mediate evolutionary change.

Phenotypic plasticity: The capacity of an individual organism to alter its features in direct response to changes in environmental conditions; sometimes used synonymously with developmental plasticity. Although this capacity is often assumed to be adaptive (i.e., enhance the individual's fitness), it need not be.

Plasticity-led evolution: An evolutionary process that occurs when a change in the environment triggers a change in phenotype via phenotypic plasticity in an ancestral lineage, and this pre-existing plasticity is subsequently refined by selection into an adaptive phenotype in a derived lineage; sometimes referred to as plasticity-first evolution. Reaction norm: A graphical representation of the set of phenotypes that a single genotype produces in response to some specific environmental variable(s); individuals show plasticity if their reaction norm is nonhorizontal.

Sexual signals: Behaviors, ornaments, or other traits that enhance mating success with the choosing sex in mating-related contexts. 


\section{Box 1: A Context Where Offspring Plasticity is Likely to Serve as a Fitness Benefit of Mate Choice.}

Many of the conditions that favor offspring plasticity as a benefit of mate choice are especially likely to arise among species with complex life histories, where juvenile and adult life stages are separated by a major developmental event—metamorphosis [75]. This developmental mode is predominant among animals [75], and animals with complex life histories (e.g., arthropods, fish, and amphibians) are frequently used to study mate choice and its fitness consequences $[6,8]$. A key feature of complex life histories is that parents and offspring express distinct phenotypes that are adapted to different ecological circumstances [75]. Moreover, in species with complex life histories, plasticity appears to be more commonly expressed in early — as opposed to later-life stages (e.g., $[40,76,77])$ possibly because adult environments are less variable than those of juveniles or because adults are more constrained in their ability to express plasticity than are juveniles [48, 78-80]. Regardless of why plasticity is more likely in juveniles, species with complex life histories provide particularly good candidate systems for evaluating whether plasticity can serve as a fitness benefit of mate choice.

\section{Outstanding Questions}

1. What are the genetic, developmental, and evolutionary mechanisms by which offspring plasticity become associated with adult sexual traits? Addressing this question is fundamental to our understanding of whether and how plasticity serves as a fitness benefit of mate choice.

2. Are there particular traits, taxa or conditions in which sexual traits are more likely to predict offspring plasticity? Evaluating these factors would provide insights into the processes by which plasticity serves as a fitness benefit of mate choice. 
3. Can mate choice for offspring plasticity explain some of the conflicting evidence for indirect benefits of mate choice? If offspring plasticity commonly mediates connections between sexual traits and offspring fitness, then past tests of indirect benefits might have failed to capture important dimensions of how these models apply to natural populations (e.g., by rearing offspring in only one environment).

4. Can sexual selection, paradoxically, lead to the loss of plasticity? If offspring plasticity is associated with adult sexual traits, then any changes to those traits could result in the production of less-plastic offspring and indirectly facilitate the loss of plasticity, a process known as genetic assimilation. Such changes to mating traits might not arise because offspring plasticity is selectively disfavored per se. Instead, selection might favor mating traits that maximize other components of fitness that are under stronger selection. 
Table 1. General conditions favoring the evolution of mate preferences for individuals that produce adaptively plastic offspring.

\begin{tabular}{|c|c|c|}
\hline Condition & Reason the condition matters & Situations in which condition might arise \\
\hline $\begin{array}{l}\text { Spatially or temporally variable } \\
\text { environment that is predictable, } \\
\text { owing to salient, reliable cues to } \\
\text { impending or prevailing } \\
\text { environmental conditions. }\end{array}$ & $\begin{array}{l}\text { Favors the evolution of plasticity in general }[39,40 \text {, } \\
81] \text {. }\end{array}$ & Any varying environment in which predictive cues occur. \\
\hline $\begin{array}{l}\text { Offspring fitness is frequency- } \\
\text { dependent. }\end{array}$ & $\begin{array}{l}\text { Parents cannot predict which offspring traits will be } \\
\text { favored (because it will depend on what traits are } \\
\text { assumed by others in the offspring's generation). }\end{array}$ & $\begin{array}{l}\text { Competitive situations, which can generate negative } \\
\text { frequency-dependent disruptive selection [82]. }\end{array}$ \\
\hline $\begin{array}{l}\text { Reliable cues about the environment } \\
\text { unavailable to parents or are more } \\
\text { effectively assessed by offspring. }\end{array}$ & $\begin{array}{l}\text { Parents cannot predict which offspring traits will be } \\
\text { favored if cues are unavailable to them }[4,21] \text {. } \\
\text { Plasticity in the offspring versus the parents is } \\
\text { favored if offspring can better assess environmental } \\
\text { cues [sensu } 39,40,81] \text {. }\end{array}$ & $\begin{array}{l}\text { Any system where the parents' and offspring's environment } \\
\text { are decoupled because adults mate in times or locations that } \\
\text { differ from where offspring develop. This includes: } \\
\text { (1) systems in which adults mate in a different season } \\
\text { or location from when or where offspring develop } \\
\text { (e.g., [40,76, 77]); } \\
\text { (2) species with complex life histories in which adults } \\
\text { and offspring inhabit disparate niches (e.g., aquatic } \\
\text { and terrestrial) [75]. }\end{array}$ \\
\hline $\begin{array}{l}\text { Parents' ability to express plasticity } \\
\text { is too costly or limited relative to } \\
\text { costs or limits of plasticity in } \\
\text { offspring [sensu 83]. }\end{array}$ & $\begin{array}{l}\text { Favors the evolution of plasticity in offspring as } \\
\text { opposed to the parents. }\end{array}$ & $\begin{array}{l}\text { Any system where plasticity is differentially favored or is } \\
\text { differentially likely to evolve in parents versus offspring, } \\
\text { including: } \\
\text { (1) taxa with complex life histories in which plasticity } \\
\text { is favored only in early life stages (e.g., [40, 76, } \\
77] \text { ); } \\
\text { (2) taxa in which early_-but not late_expression of } \\
\text { plasticity is required for the development of } \\
\text { optimal trait integration [48, 78-80]; } \\
\text { (3) taxa in which adults have few, if any, opportunities } \\
\text { for multiple matings (such systems would be less } \\
\text { likely to evolve plasticity in sexual traits) [6, 8]; } \\
\text { (4) systems in which sexual traits are under } \\
\text { countervailing selective pressures that favor } \\
\text { constitutive--as opposed to plastic-expression [6, } \\
\text { 8]. }\end{array}$ \\
\hline
\end{tabular}


Table 2. Evidence of genetic variation in plasticity as well as of sexual selection in selected animals. All examples show plasticity prior to sexual maturity.

\begin{tabular}{|c|c|c|c|c|c|c|c|c|}
\hline \multirow[b]{2}{*}{ Taxon } & \multicolumn{5}{|c|}{ Evidence of plasticity exhibiting $\mathbf{G} \times \mathbf{E}$} & \multicolumn{3}{|c|}{ Evidence of sexual selection } \\
\hline & Trait(s) & $\begin{array}{l}\text { Stimulus/ } \\
\text { source of } \\
\text { environmental } \\
\text { variation }\end{array}$ & $\begin{array}{l}\text { Fitness } \\
\text { enhancing? }\end{array}$ & $\begin{array}{l}\text { Level of } \\
G \times E\end{array}$ & $\operatorname{Ref}(\mathbf{s})$ & Mate choice & Sexual signals & $\operatorname{Ref}(\mathbf{s})$ \\
\hline $\begin{array}{l}\text { Cladocera: } \\
\text { water flea, } \\
\text { Daphnia pulex }\end{array}$ & $\begin{array}{l}\text { Size and age } \\
\text { at maturity }\end{array}$ & $\begin{array}{l}\text { Predator cue } \\
\text { concentration }\end{array}$ & Possibly & $\begin{array}{l}\text { Sibship, } \\
\text { population }\end{array}$ & [84] & $\begin{array}{l}\text { Cyclically } \\
\text { parthenogenetic; whether } \\
\text { and how mate choice } \\
\text { occurs is uncertain; } \\
\text { females flee from males } \\
\text { that pursue them, } \\
\text { possibly imposing } \\
\text { selection on male pursuit } \\
\text { ability }\end{array}$ & $\begin{array}{l}\text { Unclear; males pursue } \\
\text { females }\end{array}$ & [85] \\
\hline $\begin{array}{l}\text { Insect } \\
\text { (Diptera): } \\
\text { fruit fly, } \\
\text { Drosophila } \\
\text { melanogaster }\end{array}$ & $\begin{array}{l}\text { Body size and } \\
\text { wing length }\end{array}$ & Temperature & Possibly & $\begin{array}{l}\text { Sibship, } \\
\text { population }\end{array}$ & [86] & $\begin{array}{l}\text { Mutual mate choice; } \\
\text { males preferentially mate } \\
\text { with larger females; } \\
\text { females choose mates } \\
\text { via a combination of } \\
\text { male signals }\end{array}$ & $\begin{array}{l}\text { Males express visual, } \\
\text { auditory, and tactile } \\
\text { signals in the course of } \\
\text { "courtship dances" and } \\
\text { associated mating } \\
\text { behaviors }\end{array}$ & [87] \\
\hline $\begin{array}{l}\text { Insect } \\
\text { (Diptera): } \\
\text { yellow dung } \\
\text { fly, } \\
\text { Scathophaga } \\
\text { stercoraria }\end{array}$ & $\begin{array}{l}\text { Rates of } \\
\text { growth and } \\
\text { development, } \\
\text { body size }\end{array}$ & $\begin{array}{l}\text { Resource } \\
\text { availability, } \\
\text { conspecific } \\
\text { density }\end{array}$ & Yes & Sibship & [88] & $\begin{array}{l}\text { Female choice is } \\
\text { primarily cryptic and } \\
\text { based on male genotypes } \\
\text { at the } \\
\text { phosphoglucomutase } \\
\text { locus }\end{array}$ & $\begin{array}{l}\text { Male } \\
\text { phosphoglucomutase } \\
\text { genotype may be signaled } \\
\text { by hydrocarbons }\end{array}$ & [89] \\
\hline $\begin{array}{l}\text { Insect } \\
\text { (Lepidoptera): } \\
\text { squinting bush } \\
\text { brown } \\
\text { butterfly, } \\
\text { Bicyclus } \\
\text { anynana }\end{array}$ & $\begin{array}{l}\text { Wing } \\
\text { morphology } \\
\text { and color }\end{array}$ & $\begin{array}{l}\text { Developmental } \\
\text { temperature }\end{array}$ & Yes & $\begin{array}{l}\text { Sibship, } \\
\text { sex, } \\
\text { population }\end{array}$ & [90] & $\begin{array}{l}\text { Females choose mates } \\
\text { on the basis of a } \\
\text { complex set of male } \\
\text { mating traits }\end{array}$ & $\begin{array}{l}\text { Males perform ritualized } \\
\text { courtship behaviors, emit } \\
\text { sex pheromones, and } \\
\text { advertise wing pattern, } \\
\text { coloration, and size to } \\
\text { attract females }\end{array}$ & [91] \\
\hline
\end{tabular}




\begin{tabular}{|c|c|c|c|c|c|c|c|c|}
\hline $\begin{array}{l}\text { Insect } \\
\text { (Blattodea): } \\
\text { Chinese } \\
\text { cockcroach, } \\
\text { Eupolyphaga } \\
\text { sinensis }\end{array}$ & $\begin{array}{l}\text { Rates of } \\
\text { growth and } \\
\text { development }\end{array}$ & $\begin{array}{l}\text { Developmental } \\
\text { temperature }\end{array}$ & Possibly & $\begin{array}{l}\text { Sex, } \\
\text { population }\end{array}$ & [92] & $\begin{array}{l}\text { Unclear; exhibits sexual } \\
\text { size dimorphism in } \\
\text { which females are } \\
\text { always larger }\end{array}$ & $\begin{array}{l}\text { Like most cockroaches, } \\
\text { females emit sex } \\
\text { pheromones to attract } \\
\text { males }\end{array}$ & {$[93,94]$} \\
\hline $\begin{array}{l}\text { Insect } \\
\text { (Coleoptera): } \\
\text { Asian lady } \\
\text { beetle, } \\
\text { Harmonia } \\
\text { axyridis }\end{array}$ & $\begin{array}{l}\text { Development } \\
\text { time, adult } \\
\text { size }\end{array}$ & $\begin{array}{l}\text { Novel trophic } \\
\text { resource }\end{array}$ & Possibly & Sibship & [95] & $\begin{array}{l}\text { Females preferentially } \\
\text { mate with males with } \\
\text { bright elytra coloration }\end{array}$ & Elytra coloration & [96] \\
\hline $\begin{array}{l}\text { Insect } \\
\text { (Coleoptera): } \\
\text { leaf beetle, } \\
\text { Oreina spp. }\end{array}$ & $\begin{array}{l}\text { Development } \\
\text { time, growth }\end{array}$ & $\begin{array}{l}\text { Host-plant } \\
\text { fungal infection }\end{array}$ & Yes & $\begin{array}{l}\text { Population, } \\
\text { species }\end{array}$ & [97] & $\begin{array}{l}\text { Females choose mates } \\
\text { on the basis of chemical- } \\
\text { defense production and } \\
\text { body size; males } \\
\text { preferentially mate with } \\
\text { large females }\end{array}$ & $\begin{array}{l}\text { Male indicators of } \\
\text { chemical-defense } \\
\text { production unclear }\end{array}$ & [98] \\
\hline $\begin{array}{l}\text { Insect } \\
\text { (Coleoptera): } \\
\text { seed beetle, } \\
\text { Stator } \\
\text { limbatus }\end{array}$ & $\begin{array}{l}\text { Survivorship, } \\
\text { body size, } \\
\text { female } \\
\text { fecundity }\end{array}$ & $\begin{array}{l}\text { Developmental } \\
\text { temperature }\end{array}$ & Possibly & Population & [99] & $\begin{array}{l}\text { Females prefer large } \\
\text { males }\end{array}$ & $\begin{array}{l}\text { Unclear whether females } \\
\text { assess anything other than } \\
\text { male body size }\end{array}$ & {$[100]$} \\
\hline $\begin{array}{l}\text { Insect } \\
\text { (Hymenoptera } \\
\text { ): } \\
\text { Ichneumon } \\
\text { wasp, } \\
\text { Venturia } \\
\text { canescens }\end{array}$ & $\begin{array}{l}\text { Body size, } \\
\text { development } \\
\text { rate, } \\
\text { fecundity }\end{array}$ & $\begin{array}{l}\text { Developmental } \\
\text { temperature }\end{array}$ & Possibly & Population & [101] & $\begin{array}{l}\text { Males preferentially } \\
\text { mate with non-kin }\end{array}$ & $\begin{array}{l}\text { Females emit sex } \\
\text { pheromones that attract } \\
\text { males }\end{array}$ & $\begin{array}{l}{[102,} \\
103]\end{array}$ \\
\hline $\begin{array}{l}\text { Echinodermata } \\
: \\
\text { pebble- } \\
\text { collector } \\
\text { urchin, } \\
\text { Pseudoboletia } \\
\text { Indiana, and } \\
\text { crown of } \\
\text { thorns starfish, }\end{array}$ & $\begin{array}{l}\text { Embryonic } \\
\text { and larval } \\
\text { acidity and } \\
\text { thermal } \\
\text { tolerances }\end{array}$ & $\begin{array}{l}\text { Ocean } \\
\text { acidification } \\
\text { and warming }\end{array}$ & Yes & Sibship & $\begin{array}{l}{[104,} \\
105]\end{array}$ & $\begin{array}{l}\text { Gamete-mediated mate } \\
\text { choice via bindin-protein } \\
\text { recognition }\end{array}$ & $\begin{array}{l}\text { No reported adult sexual } \\
\text { signals }\end{array}$ & {$[106]$} \\
\hline
\end{tabular}




\begin{tabular}{|c|c|c|c|c|c|c|c|c|}
\hline $\begin{array}{l}\text { Acanthaster } \\
\text { placi }\end{array}$ & & & & & & & & \\
\hline $\begin{array}{l}\text { Fish: } \\
\text { three-spined } \\
\text { stickleback, } \\
\text { Gasterosteus } \\
\text { aculeatus }\end{array}$ & $\begin{array}{l}\text { Larval and } \\
\text { juvenile } \\
\text { growth rate, } \\
\text { survival }\end{array}$ & $\begin{array}{l}\text { Salinity of } \\
\text { developmental } \\
\text { environment }\end{array}$ & Yes & $\begin{array}{l}\text { Sibship, } \\
\text { population }\end{array}$ & [107] & $\begin{array}{l}\text { Females choose mates } \\
\text { on the basis of several } \\
\text { male traits }\end{array}$ & $\begin{array}{l}\text { Males advertise red } \\
\text { coloration, courtship } \\
\text { dances, and nest quality to } \\
\text { attract females }\end{array}$ & $\begin{array}{l}108, \\
109]\end{array}$ \\
\hline $\begin{array}{l}\text { Fish: } \\
\text { pumpkinseed, } \\
\text { Lepomis } \\
\text { gibbosus }\end{array}$ & $\begin{array}{l}\text { Body shape, } \\
\text { trophic } \\
\text { morphology }\end{array}$ & $\begin{array}{l}\text { Interspecific } \\
\text { competition, } \\
\text { resource } \\
\text { availability, } \\
\text { microhabitat } \\
\text { use }\end{array}$ & Yes & $\begin{array}{l}\text { Sibship, } \\
\text { population }\end{array}$ & [110] & $\begin{array}{l}\text { Females are attracted to } \\
\text { male nest sites; specific } \\
\text { signals are unclear, but } \\
\text { possibly a combination } \\
\text { of male traits and nest } \\
\text { characteristics }\end{array}$ & $\begin{array}{l}\text { Specific signals are } \\
\text { unclear, but males attract } \\
\text { females to their nests, } \\
\text { which are concentrated in } \\
\text { shallow waters }\end{array}$ & $\begin{array}{l}{[111,} \\
112]\end{array}$ \\
\hline $\begin{array}{l}\text { Fish: } \\
\text { mouthbrooder } \\
\text { cichlid, } \\
\text { Pseudocrenila } \\
\text { brus } \\
\text { multicolor } \\
\text { victoriae }\end{array}$ & Brain mass & $\begin{array}{l}\text { Dissolved } \\
\text { oxygen in } \\
\text { developmental } \\
\text { environment }\end{array}$ & Yes & $\begin{array}{l}\text { Sibship, } \\
\text { population }\end{array}$ & [113] & $\begin{array}{l}\text { Female select mates on } \\
\text { the basis of courtship } \\
\text { displays and fin } \\
\text { coloration }\end{array}$ & $\begin{array}{l}\text { Males attract females via } \\
\text { "mating wiggle" displays } \\
\text { and "egg spots" on anal } \\
\text { fins }\end{array}$ & [114] \\
\hline $\begin{array}{l}\text { Fish: } \\
\text { Japanese rice } \\
\text { fish, } \\
\text { Oryzias latipes }\end{array}$ & Growth rate & $\begin{array}{l}\text { Developmental } \\
\text { temperature }\end{array}$ & Possibly & $\begin{array}{l}\text { Sibship, } \\
\text { population }\end{array}$ & [115] & $\begin{array}{l}\text { Females choose mates } \\
\text { on the basis of secondary } \\
\text { sexual characters and } \\
\text { courtship behaviors }\end{array}$ & $\begin{array}{l}\text { Males advertise fin } \\
\text { ornaments and perform } \\
\text { courtship behaviors }\end{array}$ & $\begin{array}{l}{[116,} \\
117]\end{array}$ \\
\hline $\begin{array}{l}\text { Fish: } \\
\text { crimson- } \\
\text { spotted } \\
\text { rainbowfish, } \\
\text { Melanotaenia } \\
\text { duboulayi } \\
\end{array}$ & $\begin{array}{l}\text { Gene } \\
\text { expression at } \\
\text { loci involved } \\
\text { in responses } \\
\text { to } \\
\text { temperature }\end{array}$ & $\begin{array}{l}\text { Developmental } \\
\text { temperature }\end{array}$ & Possibly & Sibship & [118] & $\begin{array}{l}\text { Females prefer males } \\
\text { with high display rates } \\
\text { and most commonly } \\
\text { mate with dominant, } \\
\text { large, bold males }\end{array}$ & $\begin{array}{l}\text { Males compete for } \\
\text { dominance, and dominant } \\
\text { males exhibit active, bold } \\
\text { behavioral syndromes and } \\
\text { high display rates }\end{array}$ & $\begin{array}{l}{[119,} \\
120]\end{array}$ \\
\hline 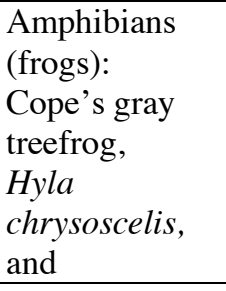 & $\begin{array}{l}\text { Tadpole tail } \\
\text { morphology, } \\
\text { foraging } \\
\text { behavior }\end{array}$ & Predation cues & Yes & $\begin{array}{l}\text { Sibship, } \\
\text { population }\end{array}$ & $\begin{array}{l}{[121-} \\
123]\end{array}$ & $\begin{array}{l}\text { Females choose mates } \\
\text { on the basis of call } \\
\text { characteristics }\end{array}$ & $\begin{array}{l}\text { Males form aggregations } \\
\text { and call to attract females }\end{array}$ & $\begin{array}{l}{[124,} \\
125]\end{array}$ \\
\hline
\end{tabular}




\begin{tabular}{|c|c|c|c|c|c|c|c|c|}
\hline $\begin{array}{l}\text { eastern gray } \\
\text { treefrog, } \\
\text { H.versicolor }\end{array}$ & & & & & & & & \\
\hline $\begin{array}{l}\text { Amphibian } \\
\text { (frog): } \\
\text { wood frog, } \\
\text { Lithobates } \\
\text { sylvaticus } \\
\text { (Rana } \\
\text { sylvatica) }\end{array}$ & $\begin{array}{l}\text { Tadpole tail } \\
\text { morphology, } \\
\text { foraging } \\
\text { behavior }\end{array}$ & Predation cues & Yes & $\begin{array}{l}\text { Sibship, } \\
\text { population }\end{array}$ & {$[126]$} & $\begin{array}{l}\text { Sexual selection driven } \\
\text { primarily by scramble } \\
\text { competition among } \\
\text { males, but males } \\
\text { preferentially mate with } \\
\text { relatively large females }\end{array}$ & $\begin{array}{l}\text { Males form aggregations } \\
\text { and call to attract females }\end{array}$ & {$[127]$} \\
\hline $\begin{array}{l}\text { Amphibian } \\
\text { (frog): } \\
\text { moor frog, } \\
\text { Rana arvalis }\end{array}$ & $\begin{array}{l}\text { Tadpole } \\
\text { acidity } \\
\text { tolerance }\end{array}$ & $\begin{array}{l}\text { Pond } \\
\text { acidification }\end{array}$ & Yes & $\begin{array}{l}\text { Sibship, } \\
\text { population }\end{array}$ & {$[128]$} & $\begin{array}{l}\text { Sexual selection driven } \\
\text { primarily by scramble } \\
\text { competition among } \\
\text { males, but females } \\
\text { preferentially mate with } \\
\text { males with high } \\
\text { fertilization success }\end{array}$ & $\begin{array}{l}\text { Males form aggregations } \\
\text { and call to attract females; } \\
\text { trait(s) associated with } \\
\text { male fertilization success } \\
\text { unknown }\end{array}$ & {$[129]$} \\
\hline $\begin{array}{l}\text { Amphibian } \\
\text { (salamander): } \\
\text { slender } \\
\text { salamanders, } \\
\text { Batrachoseps } \\
\text { spp. }\end{array}$ & $\begin{array}{l}\text { Number of } \\
\text { trunk } \\
\text { vertebrae }\end{array}$ & $\begin{array}{l}\text { Developmental } \\
\text { temperature }\end{array}$ & Possibly & $\begin{array}{l}\text { Sibship, } \\
\text { population, } \\
\text { species }\end{array}$ & {$[130]$} & $\begin{array}{l}\text { Unclear, but pheromones } \\
\text { are implicated at least at } \\
\text { the level of species } \\
\text { recognition }\end{array}$ & Possibly pheromones & {$[131]$} \\
\hline
\end{tabular}




\section{CHAPTER 3: MALE SEXUAL SIGNAL PREDICTS PHENOTYPIC PLASTICITY IN OFFSPRING: IMPLCATIONS FOR THE EVOLUTION OF PLASTICITY AND LOCAL ADAPTATION}

The following is adapted from:

Kelly, P.W., Pfennig, D.W., de la Serna, S., and Pfennig, K.S. 2019. Male sexual signal

predicts phenotypic plasticity in offspring: implications for the evolution of plasticity and

local

adaptation. Philosophical Transactions of the Royal Society B. 374: 20180179.

\section{Introduction}

In an ever-changing world, environmental fluctuations preclude any one phenotype from being consistently favored by natural selection [36]. To cope with these fluctuations, organisms have evolved numerous strategies to generate adaptive phenotypic variation [132]. One such widely used strategy is phenotypic plasticity (hereafter, simply 'plasticity')— the ability of an individual organism to change its phenotype in direct response to stimuli or inputs from the environment [7, 133]. Although not all plasticity is adaptive [134], 'adaptive plasticity' (i.e., plasticity that enhances the bearer's fitness [135]) enables organisms to respond to environmental variability by expressing phenotypes that are selectively favored under prevailing conditions, even when these conditions change unpredictably $[7,136]$. An open question, however, is whether and how plasticity impacts evolution. On the one hand, some researchers argue that plasticity only impedes evolution [137]. According to this viewpoint, if a single genotype can produce multiple phenotypes in response to changing 
environmental conditions, then such plasticity should shield populations from strong directional selection [138]. On the other hand, a growing number of researchers maintain that plasticity can play a leading and decisive role in evolution [7, 40, 139-150]. For example, if underlying genetic variation exists in either the tendency or manner in which individuals respond to the environment (i.e., if different genotypes exhibit different 'reaction norms,' as is nearly always the case $[151,152])$, then selection can act on this variation-revealed to selection through plasticity — and refine the expression of the affected trait such that it is optimally suited to local conditions [7]. In this way, plasticity can facilitate local adaptation [153-155].

Like plasticity, sexual selection —-the differential mating success of individuals stemming from competition for mates [6] — has been proposed to both facilitate and impede adaptive evolution $[6,156-158]$. Although there are numerous ways in which sexual selection can promote adaptation [158], it has long been regarded as an impediment to adaptive evolution in rapidly changing environments $[72,159]$. In such situations, sexual selection can generate an evolutionary momentum that maintains patterns of mating behaviours that, while they may have enhanced local adaptation to some past environment, result in the production of maladaptive offspring in the altered environment (Fig. 1a). In other words, in everchanging environments, females might often harbour preferences for males who sire offspring that are locally disfavored by natural selection. Although these females would likely be under strong selection to adopt new preferences for males who produce offspring that are favored in the changed environment $[160,161]$, there will likely be a time lag before such selection promotes the evolution of either new preferences or the sexual signals that indicate a male's ability to sire offspring that are adaptive in the altered environment. 


\section{During this time lag, populations might have lower mean fitness and even a higher risk of}

\section{extinction $[162,163]$. Moreover, populations in constantly changing environments might be}

a) Time 1: Females should possess preferences for males who sire offspring with locally adapted traits.

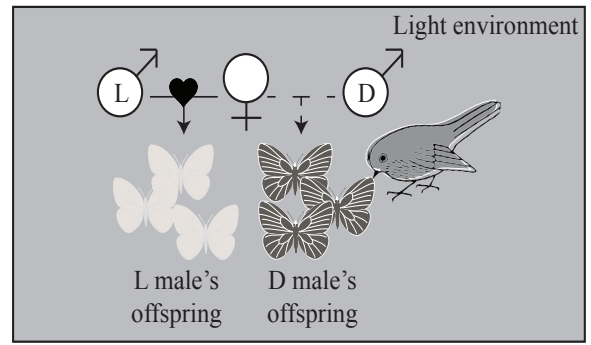

Time 2: If the environment changes rapidly, a formerly adaptive preference can become maladaptive.
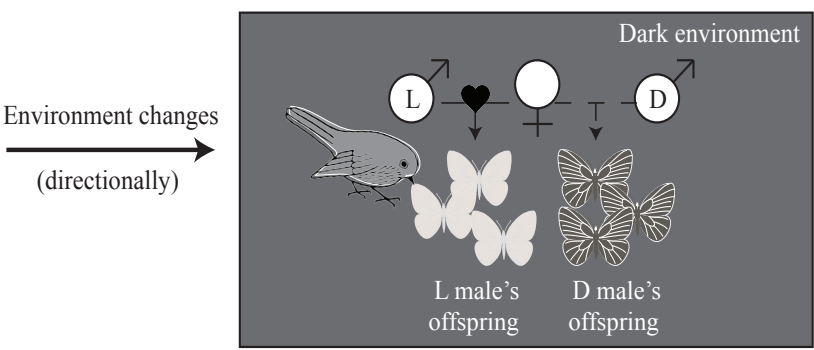

b)

In variable environments, females that prefer males who sire plastic offspring ('P' males) should produce more surviving offspring — across all environments - than females that prefer males who sire non-plastic offspring ('L' or 'D' males).
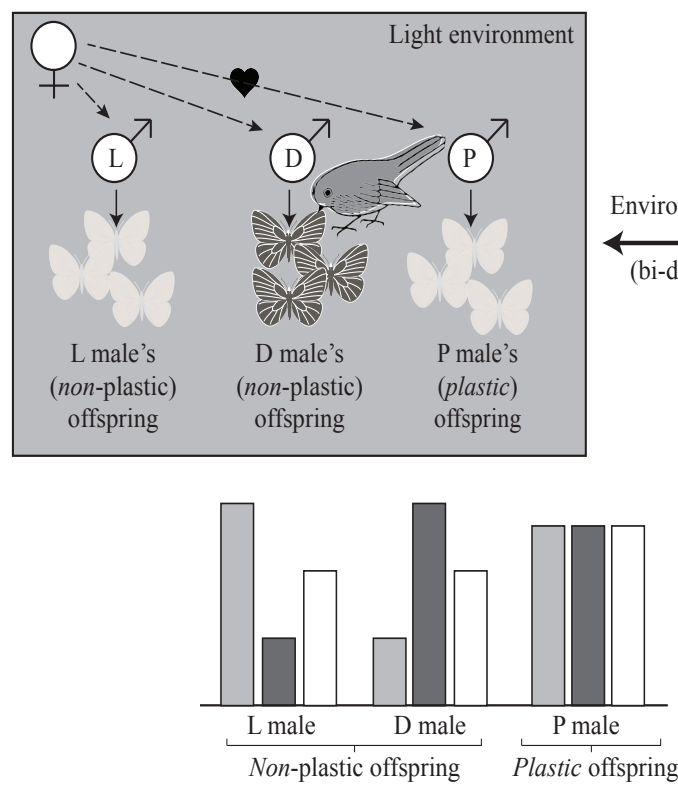

Environment changes
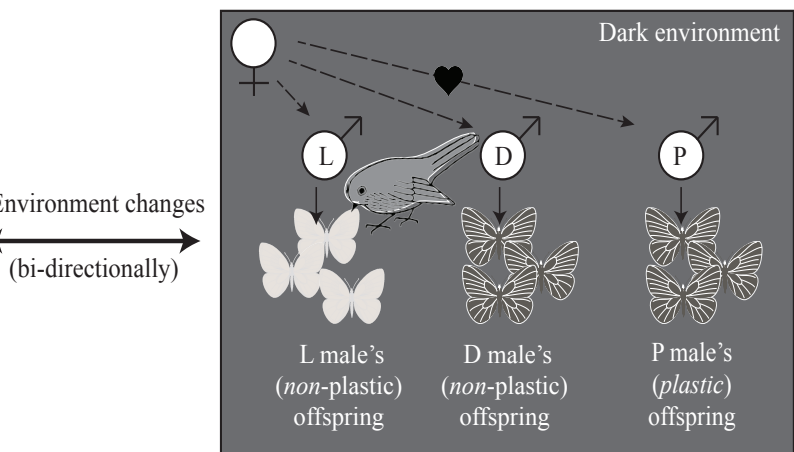

Number of surviving offspring produced by each type of male:

in Light environment

in Dark environment

averaged across Both environments

\section{Figure 1.}

a) How sexual selection (as mediated by female mate choice) impedes local adaptation in rapidly changing environments. Generally, females should possess preferences for males who sire offspring with locally adapted traits. For instance, females that occur in a light environment in which visually oriented predators are present should prefer males (' $\mathrm{L}$ ' males) who sire light-colored offspring over males ('D' males) who sire dark-colored offspring. However, in a rapidly changing environment (in this case, one that changes into a dark environment), a formerly adaptive preference might become maladaptive. Here, the formerly adaptive light-colored offspring sired by the ' $\mathrm{L}$ ' male are now more likely to be detected by predators. b) When females preferentially choose males that sire plastic offspring, sexual selection can facilitate local adaptation to rapidly changing environments. In this case, males that sire plastic offspring (' $\mathrm{P}$ ' males) should produce more surviving offspring than either L or D males (who sire non-plastic offspring) across both environments (note that we have assumed here that plastic offspring bear a cost not borne by non-plastic offspring in the environment for which they are adapted; weakening this assumption only increases the advantage to P males). 
perpetually in this state of 'evolutionary time lag,' in which females continually express preferences for mates who sire offspring that are maladapted to the prevailing environment, thereby causing sexual selection to inhibit local adaptation.

These arguments assume that females have fixed preferences. However, individual females can modify their mate choice preferences in direct response to prevailing conditions $[8,164,165]$. These 'context-dependent preferences' (sensu [165]) are a form of plasticity, and they allow females to choose mates who sire offspring with adaptive traits, even in rapidly changing environments. Such plasticity is widespread, and it is expected to evolve if females can detect cues from the environment that reliably predict the environmental context that their offspring will experience $[8,165$, e.g., 166].

However, females cannot always reliably assess the environment that their offspring will encounter. Such a situation might be especially likely to arise when the environment changes rapidly (i.e., within an individual's lifetime) and unpredictably. In these circumstances, females could produce adaptive offspring by preferring males who sire offspring that are themselves plastic. In particular, selection could favor females that prefer mates who produce offspring that can assess and respond adaptively to their local environment through plasticity. As a consequence of such preferences, sexual selection would facilitate local adaptation. Moreover, because plasticity is typically favored in variable environments [7, 136], sexual selection (specifically, mate choice) could also operate in tandem with natural selection to promote the evolution of plasticity. This notion that mate choice itself might promote plasticity is especially intriguing. Indeed, although researchers have long recognized that sexual selection can shape the evolution of plasticity via multiple routes (e.g., by favoring the evolution of condition-dependent female 
preferences or male signals; Table 1), the possibility that sexual selection might favor plasticity's origin and maintenance has rarely been considered. Yet, clarifying whether sexual selection can favor the evolution of plasticity is important, not only for understanding the conditions under which sexual selection can facilitate local adaptation in variable environments, but also for understanding how plasticity itself evolves.

Here, we evaluated these ideas empirically by using spadefoot toads, Spea multiplicata, as our model system. As we explain below, in this species, female mate choice mediates sexual selection on male sexual signals [167-169]. Moreover, the offspring develop in highly variable environments that favor plasticity; consequently, the tadpoles produce a novel environmentally induced resource-use phenotype as an alternative to their default tadpole morphology $[3,27,170]$. Using an experimental approach, we specifically evaluated whether: 1) a male sexual signal (that is known to be preferred by females) predicts the degree and quality of expression of the induced resource-use morphology; 2) the preferred male sexual signal predicts plasticity in the production of the induced morphology versus the alternative default morphology; and 3) the preferred male sexual signal predicts offspring fitness such that more plastic offspring have enhanced fitness relative to less-plastic offspring.

Our results indicate that the preferred male sexual signal does indeed predict: the expression of environmentally induced resource-use morphology in offspring; the extent of plasticity in that morphology versus the default morphology; and the resulting fitness of that plasticity. Critically, females are known to prefer males with the sexual signals that we found are indicative of greater plasticity and higher offspring fitness. Consequently, our results suggest 
that sexual selection can facilitate adaptation by promoting the evolution of plasticity.

\section{Materials and Methods}

\section{(a) Study System and Specific Goals}

Mexican spadefoot toads, Spea multiplicata, occur in the desert scrub and desert grasslands of northern Mexico and the southwestern USA. In these regions, annual rainfall is concentrated in July and August, when localized thunderstorms fill highly ephemeral ponds that vary in depth and duration, both spatially and temporally $[3,27,166]$. Spadefoot toads breed, and their tadpoles develop, in these ephemeral ponds.

Spea breed on a single night following pond filling $[167,171]$. Males call to attract mates, and females choose their mates by initiating pair formation when they closely approach and touch the male [167]. Sexual signalling and mate choice in any one population take place over a few hours; thus, mate choice is not confounded by variation in which males attend an aggregation on a given night or by nightly variation in environmental factors such as temperature $[167,171]$.

In populations where they do not risk hybridizing with a congener ( $S$. bombifrons), female $S$. multiplicata prefer males with faster call rates that are in better condition [167-169, 172]. By preferring such males, females choose mates that provide them with higher fertilization success and enhanced growth in offspring $[167,168]$.

Spea multiplicata tadpoles express alternative, environmentally induced resource-use phenotypes $[3,27]$. Specifically, tadpoles develop into either an 'omnivore' ecomorph, a round-bodied tadpole with a long intestine, small jaw muscles, and smooth keratinized mouthparts, or a 'carnivore' ecomorph, a narrow-bodied tadpole with a short intestine, greatly enlarged jaw muscles, and serrated mouthparts. Omnivores are dietary generalists that 
feed on detritus, algae, and small crustaceans; carnivores are dietary specialists that feed mostly on anostracan fairy shrimp and other tadpoles [173].

Development of these alternative ecomorphs is underlain by phenotypic plasticity. Spea tadpoles are born as omnivores, but if a young individual consumes fairy shrimp or other tadpoles, it may develop into the carnivore phenotype [27, 174]. However, families differ in propensity to produce carnivores when fed shrimp or tadpoles, suggesting underlying genetic variation in ecomorph production and, hence, in the expression of phenotypic plasticity $[175,176]$. Additionally, males appear to differ in the quality of carnivore offspring that they sire as indicated by their trait integration; i.e., the nature of the correlations among different component traits of the carnivore morph [177] (see Section 2(b) below).

These alternative ecomorphs have presumably evolved as an adaptive response to pond ephemerality, resource availability, and variation therein [3, 27, 178]. Owing to pronounced variation in rainfall, resource availability, and tadpole (i.e., competitor) density across time and space, the ponds in which S. multiplicata tadpoles develop are rapidly changing, often unpredictable environments [3, 27, 178]. The carnivore ecomorph is generally favored in dry years and in ponds with high shrimp densities: by specializing on high-nutrition shrimp and tadpole prey, the carnivore ecomorph develops faster than the omnivore ecomorph, and thus is more likely to metamorphose and escape a rapidly drying pond [3, 27]. In longer-duration ponds, however, frequency-dependent selection, which arises from intraspecific competition for dietary resources, maintains both ecomorphs at an equilibrium frequency within the same pond, and this equilibrium value is set by a given a 
pond's relative availabilities of low-nutrition resources (i.e., detritus, algae, and small crustaceans) versus high-nutrition resources (i.e., shrimp and other tadpoles) [3].

Moreover, disruptive selection also acts in these ponds [179]: individuals with phenotypes that are intermediate between the two ecomorph types suffer low relative fitness, likely due to an inability to compete effectively with tadpoles expressing more extreme omnivore or carnivore phenotypes [180,181]. Thus, while the phenotypic plasticity underlying these alternative phenotypes results in continuous variation between them, almost all populations exhibit significantly bimodal distributions of the ecomorphs [178, 179]. This, in combination with competition driving frequency-dependent selection and the high variability of pond environments, favors accurate assessment of environmental cues and the capacity to produce each ecomorph (and not intermediate phenotypes) [180, 181].

Spadefoot natural history makes them particularly well-suited to evaluate if male sexual signals that are preferred by females indicate plasticity in their offspring and, concomitantly, their offspring's ability to produce locally adapted phenotypes. To do so, we carried out two experiments aimed at evaluating whether or not a male sexual signal (i.e., call rate) predicts: 1) production and quality of the environmentally induced carnivore morphology of his offspring (i.e., how carnivore-like his offspring were when fed shrimp), and 2) the degree of plasticity in resource-use morphology expressed by his offspring (i.e., how different his offspring were in carnivore versus omnivore morphology when fed shrimp versus detritus). 


\section{(b) Does a Male Sexual Signal Predict Production of an Environmentally-Induced}

\section{Phenotype in His Offspring?}

We determined if male call rate predicts the production of carnivores among his offspring when they were fed shrimp exclusively (the resource type that induces the carnivore ecomorph and for which the carnivore ecomorph is best adapted to utilize [180]). We also asked if male call rate predicts the fitness and trait integration of these carnivores. We used trait integration as a measure of the quality of carnivore phenotypes produced (see [177]).

We began by recording nine calling males at a natural breeding aggregation in an ephemeral pond ('Crater') near Portal, Arizona, USA. In this pond, S. multiplicata is the only Spea species present. The nine recorded males represented the majority of males observed calling in this aggregation, and were all recorded within an hour (so rates are not affected by differences in temperature, male assembly, or time of night). Each male was individually recorded by approaching him within $0.5 \mathrm{~m}$ using standard procedures developed for this system. After recording a male's call for at least 1 min., each male was captured with a handheld dip net and placed in an individually labelled container. The recordings were subsequently analysed for call rate; i.e., the number of calls per minute.

We then collected females from the same breeding aggregation, and on the same evening. To collect females, we used hand-held dip nets to capture amplexed pairs as they formed. We immediately broke the pairs apart to recover the female. Collecting females in this way ensured that all females in the experiment were ready to breed. Once collections were complete, we returned the animals to the nearby Southwestern Research Station and immediately paired each female at random with one of the nine recorded males. 
We placed each male-female pair in a water-filled tank and allowed them to breed naturally. The resulting eggs were aerated until tadpoles hatched. One day after hatching, we removed a subset of the tadpoles from each clutch and placed them in groups of five siblings in a microcosm (a $34 \times 20 \times 12$-cm plastic box filled with $6 \mathrm{~L}$ of dechlorinated well water). We reared tadpoles in groups to assess the propensity and degree to which each family's tadpoles expressed the carnivore phenotype (rearing tadpoles on a shrimp diet in groups induces more extreme carnivores, presumably because of competition [182]). For each family, we replicated these microcosms 24 times, so that we had a total of 192 microcosms (24 microcosms per family x 8 families) for a total starting sample size of 1080 tadpoles. Each microcosm received a unique ID for use in our analyses (see below).

On Day 1 of the experiment, we provided each microcosm with equal amounts of detritus (TetraFin ${ }^{\circledR}$ Fishfood Pellets). These pellets simulated the detritus on which $S$. multiplicata tadpoles feed in the wild [183]. After Day 1, we began the carnivore-induction feeding regimen. Specifically, on Days 2-5 of the experiment, the tadpoles in each microcosm were provided daily with $\sim 150$ small, live fairy shrimp (Streptocephalus $\mathrm{sp}$. or Thamnocephalus sp.). On Days 6-8, the tadpoles in each microcosm were provided daily with $\sim 60$ large, live fairy shrimp. All shrimp were obtained from a nearby, natural pond. On Day 9 , the tadpoles were euthanized by immersion in a $0.1 \%$ aqueous solution of tricane methanesulfonate (MS 222) and preserved in 95\% ethanol. Some tadpoles died in the experiment, and some samples were destroyed during transport, so our final sample size was 973 tadpoles.

To assess tadpole resource-use morphology, we measured the preserved tadpoles using previously published methods [181]. Specifically, we measured the width of each 
tadpole's orbitohyoideus muscle $(\mathrm{OH}$; carnivores have a larger $\mathrm{OH})$ and scored its mouthparts (MP) on an ordinal scale that ranged from 1 (most omnivorous) to 5 (most carnivorous). We also counted the number of denticle rows (DR; omnivores have higher DR counts) and gut coils (GC; omnivores have a longer gut, which manifests as higher GC counts). After correcting $\mathrm{OH}$ for body size (by regressing $\ln \mathrm{OH}$ on $\ln$ snout-vent length), we combined these four measures into a single morphological index (MI; e.g., see ref. [175] and references therein). To calculate this index, we used a principal component (PC) analysis. The MI consisted of the first principal component (the PC with an Eigen value greater than 1), which explained $51.2 \%$ of the variance in resource-use morphology among our tadpoles. Larger values of the MI correspond to more carnivore-like tadpoles, with larger $\mathrm{OH}$ muscles, fewer DR and GC, and more serrated, notched mouthparts (higher MP scores). By contrast, smaller values correspond to more omnivore-like tadpoles, with smaller $\mathrm{OH}$ muscles, higher DR and GC counts, and smoother mouthparts.

As proxies for fitness, we measured each tadpole's Gosner developmental stage (GS; [184]), body size (snout-vent length; SVL), and mass. GS is an appropriate fitness proxy, because faster-developing tadpoles are more likely to escape the highly ephemeral ponds in which S. multiplicata develop [3, 27]. SVL and mass are also appropriate fitness proxies, because larger body size is positively correlated with fitness in S. multiplicata. Larger tadpoles are more likely to survive to metamorphosis [172] and sexual maturity (D. Pfennig, unpubl. data). Larger tadpoles also mature as larger adults (D. Pfennig, unpubl. data), and adult body size, in turn, is positively correlated with mating success in males [167] and fecundity in females [172]. Finally, we measured body size of each adult male and female 
used in these breedings to account for any effects of parental body size on offspring morphology or fitness $[185,186]$.

To analyse the data, we used a linear mixed-effects model with MI as the response variable, ln call rate as the fixed effect, and family ID and replicate (microcosm) ID as random effects. The inclusion of the tadpole's parents' body size did not improve the model (likelihood ratio test, $P=0.57$ ). Therefore, parent body size was not included in subsequent analyses. To address propensities to develop as carnivores, we used a generalized linear model (GLM) with the total number of extreme carnivores (those with the highest MP scores among all tadpoles in the experiment [175]) sired by each male as the response variable and ln call rate as the predictor; we specified a Poisson distribution to account for the response consisting of count data. We also used family-level mean values of GS, SVL, and mass in regressions on male call rate to assess whether male call rate predicts the fitness of his offspring.

Finally, prior work suggests that the presence of potential competitors (i.e., individuals in the same microcosm) can impact trait integration in the form of the strength of the correlation between $\mathrm{OH}$ and GC [177]. Specifically, a stronger, more negative correlation between $\mathrm{OH}$ and $\mathrm{GC}$ indicates better trait integration because wider $\mathrm{OH}$ muscles are best suited for handling carnivore prey [175], whereas a long gut (i.e., greater GC counts) is best suited to digesting the low-nutrition omnivore diet [175]. Thus, a more-negative correlation between these two traits - for both the omnivores and carnivores - is indicative of a potentially better-performing phenotype overall $[23,33]$. Therefore, we calculated Pearson correlation coefficients for this pair of traits within each family and then regressed these family-specific coefficients on male call rate. In this latter analysis, a family with a relatively 
fast call rate was more extreme than others. An outlier analysis did not identify this family as a statistical outlier. Nevertheless, we used a non-parametric Spearman rank-order correlation analysis (which is not influenced by outliers) on the data. This non-parametric analysis produced results that were qualitatively the same as the parametric results, so we report the results from the parametric analysis.

\section{(c) Does a Male Sexual Signal Predict Adaptive Offspring Plasticity?}

The above experiment evaluated whether male signals predict the production of the carnivore morphology. However, production of the carnivore morphology among different males' offspring might be at least in part genetic rather than being entirely environmentally induced. Thus, the 'default' morph (i.e., the phenotype produced at birth) might be more carnivore-like in some families than in others. Indeed, previous studies have shown that different families vary in their propensity to produce carnivores $[175,176]$, and a related Spea species shows evidence that some tadpoles are actually born as carnivores [187]. We therefore sought to determine whether male call rate predicts plasticity in his offspring's resource-use morphology across two diets: a detritus diet (that should produce the omnivore ecomorph) and a shrimp diet (that induces the carnivore ecomorph).

We recorded 12 males at a different natural breeding aggregation than that used in section $2 \mathrm{~b}$ above. This aggregation occurred in a temporary pond ('PO2-N') between Portal, Arizona and Rodeo, New Mexico, USA. As in the experiment above, S. multiplicata was the only Spea species present, and the number of males recorded represented approximately one third of the males observed calling in this particular aggregation. Using the same procedures described above, we recorded males, paired them with females collected from their same breeding aggregation, and produced families of tadpoles. 
On the first day after the tadpoles hatched, we provided each tank with $20 \mathrm{mg}$ of detritus as above. The following day, we placed tadpoles from each clutch individually in a microcosm (a $475 \mathrm{~mL}$ plastic cup filled with $400 \mathrm{~mL}$ of dechlorinated well water; rearing tadpoles singly eliminated competition, thereby allowing us to assess each tadpole's morphological responses to dietary cues alone). We randomly selected 60 tadpoles per family and divided them between two diet treatments: one in which tadpoles received only detritus (and thus developed more omnivore-like morphology), and one in which tadpoles received only fairy shrimp (the ingestion of which can induce development of the carnivore ecomorph [27, 174]). Our starting sample size was 720 tadpoles (60 tadpoles x 12 families). Rearing cups were placed in equal-sized groups on shelves in a single room, with a shrimp-fed and a detritus-fed group from each family on each shelf (to account for any variation in temperature in the room).

Tadpoles fed detritus received 10mg of crushed fish food each on Day 1 and every second day thereafter. Shrimp-fed tadpoles received approximately 20 live fairy shrimp each on Day 1; this quantity increased by approximately 10 shrimp per day through Day 6. On Days 7-9, shrimp-fed tadpoles received live fairy shrimp ad libitum. All fairy shrimp were from natural ponds. On Day 10, we euthanized all tadpoles by immersion in MS 222 and preserved them in $95 \%$ ethanol. After some mortality, our final sample size was 706 tadpoles. Using the methods described above, we calculated a MI for each tadpole (in this experiment, MI [PC1] explained 53.4\% of variance). As above, we also measured each tadpole's GS, SVL, and mass. Additionally, we measured the body size (SVL) of the adult males and females used in the breedings to account for any effects of parental body size on offspring morphology or fitness. 
To assess the relationship between male call rate and tadpole resource-use morphology, we used a linear mixed-effects model with MI as the response variable and ln call rate, diet, and the interaction of call rate and diet as fixed effects, and family ID and group ID as random effects. The inclusion of adult body size did not improve the model (likelihood ratio test, $P=$ 0.84 ), so those measures were not included in our final analysis.

Additionally, to assess plasticity in resource-use morphology, we calculated mean MI values for each family on each diet and then subtracted each family's mean MI on the detritus (omnivore) diet from its mean MI on the shrimp (carnivore) diet. We used a linear regression of this measure of plasticity on male call rate to determine the relationship between these two variables. To assess family differences in propensities to develop as carnivores, we summed the number of extreme carnivore phenotypes (those with the highest MP score among all tadpoles reared on the shrimp diet [175]) and used a GLM with Poisson distribution specified (to account for the response being count data) with family total number of carnivores as the response variable and male call rate as the predictor variable. Finally, we used family-level mean values of GS, SVL, and mass in regressions on male call rate to assess differences in fitness proxies (for an explanation of these fitness proxies, see the description of the previous experiment above).

\section{Results}

(a) Does a Male Sexual Signal Predict Offspring Resource-use Morphology?

Faster-calling males produced significantly more carnivores than slower-calling males (GLM with a Poisson distribution; $Z$ score $=2.57, P=0.010 ;$ Fig. 2). Faster-calling 
males also tended to produce tadpoles with more extreme carnivore morphology (as measured by MI; $t$-test with Satterthwaite approximations; $t=1.90, P=0.059)$.

When we evaluated whether male call rate directly predicted offspring growth, we found no evidence that this was the case across all tadpoles (mass: $F_{1,7}=4.64, R^{2}=0.31, P=$ 0.068; SVL: $\left.F_{1,7}=3.11, P=0.121 ; \mathrm{GS}: F_{1,7}=1.48, P=0.263\right)$. Nevertheless, male call rate predicted tadpole quality in terms of trait integration. In particular, male call rate predicted the strength of a negative correlation between two key traits that impact tadpole performance: $\mathrm{OH}$ muscle size and gut length (as measured by GC). Specifically, tadpoles sired by males with faster call rates had stronger, more-negative correlations between $\mathrm{OH}$ muscle width and GC counts $\left(F_{1,7}=8.13, R^{2}=0.47, P=0.025\right)$. Moreover, the strength of this correlation between $\mathrm{OH}$ and GC predicted tadpole size (mass: $F_{1,7}=20.13, R^{2}=0.71, P=0.003$; SVL: $\left.F_{1,7}=11.6, R^{2}=0.57, P=0.011\right)$.

\section{(b) Does a Male Sexual Signal Predict Offspring Plasticity?}

Diet treatments predicted offspring growth, development, and resource-use morphology as expected. In particular, tadpoles reared on the shrimp diet grew larger, developed more quickly, and exhibited more carnivore-like phenotypes than tadpoles reared on the detritus diet (Wilcoxon rank-sum tests; SVL: $P<0.001$; mass: $P<0.001$; GS: $P<$ 0.001; MI: $P<0.001)$.

Male call rate predicted plasticity in his offspring's resource-use morphology. In particular, our linear mixed-effects model revealed a significant interaction between diet and male call rate $(t=3.02, P=0.003)$. Whereas male call rate had no relationship with offspring MI on the detritus diet $(t=-1.64, P=0.44)$, call rate positively predicted offspring MI on the shrimp diet: males with faster calls sired offspring with more extreme carnivore MI scores $(t$ 
that sexual selection can facilitate adaptation by promoting the evolution of plasticity. that sexual selection can facilitate adaptation by promoting the evolution of plasticity. that sexual selection can facilitate adaptation by promoting the evolution of plasticity. $=-3.28, P=$ 0.007; males with faster call rates also produced significantly more carnivores: $Z$ score $=$ $1.97, P=0.048)$. That faster-calling males sired more plastic tadpoles was corroborated by differences between family-mean MI scores for shrimp- and detritus-reared tadpoles: call rate positively predicted larger differences in MI between the two diets $\left(F_{1,10}=11.87, R^{2}=0.48\right.$, $P=0.007$; Fig. 3).

Across diets, we found no evidence that male call rate predicted tadpole size or growth rate (SVL: $F_{1,10}=2.52, P=0.144$; mass: $F_{1,10}=1.86, P=0.203$; GS: $F_{1,10}=0.519, P$ $=0.488$ ). However, when we excluded a single outlier family, faster-calling males produced significantly larger (but not faster developing) offspring (SVL: $F_{1,9}=10.86, R^{2}=0.50, P=$ 0.009; mass: $F_{1,9}=9.23, R^{2}=0.45, P=0.014$; GS: $\left.F_{1,9}=4.02, P=0.076\right)$. 


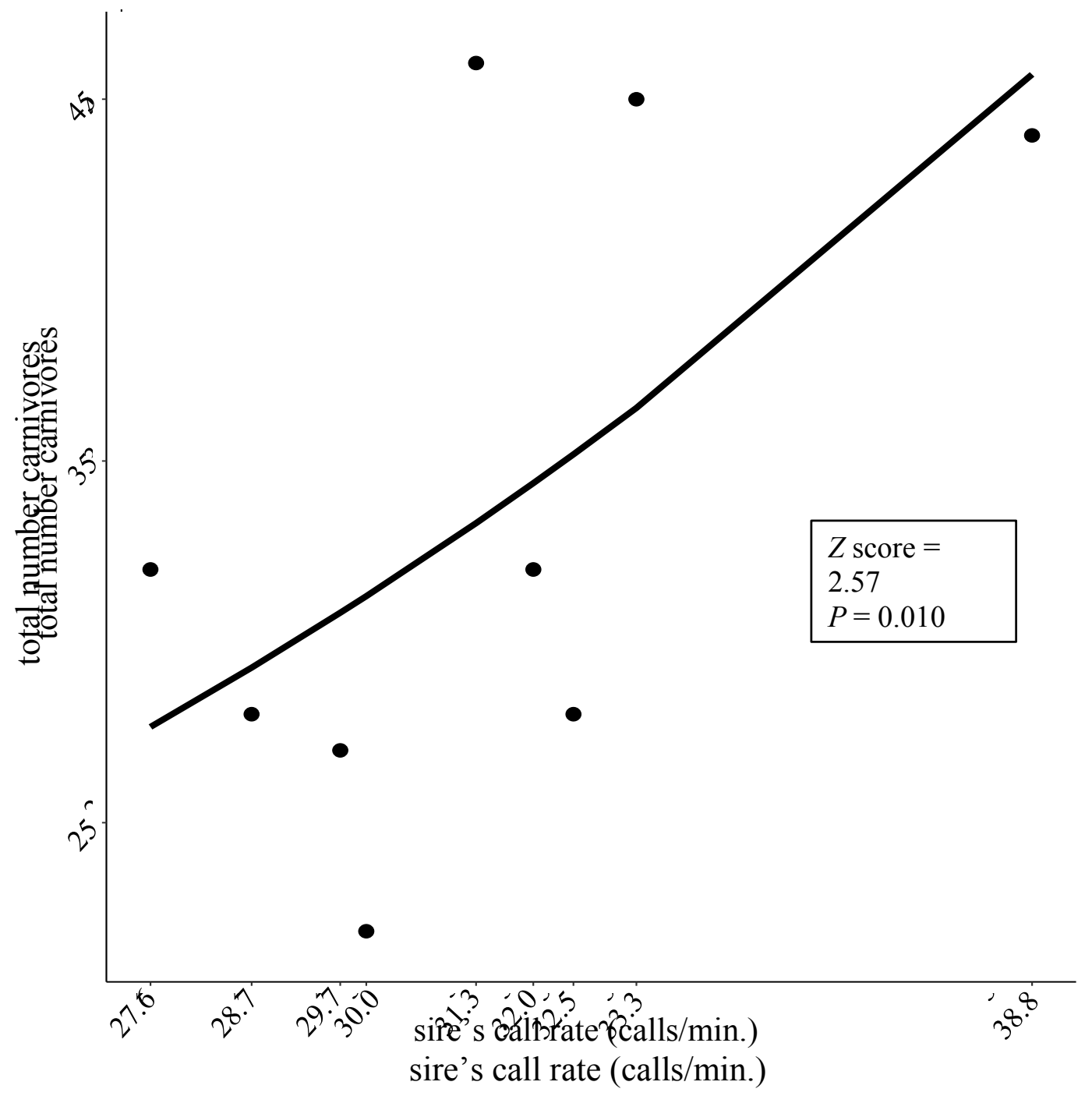

Figure 2.

Generalized linear model fit with ln call rate (back-transformed) as the predictor and total number of carnivores as the response (Poisson distribution specified). 


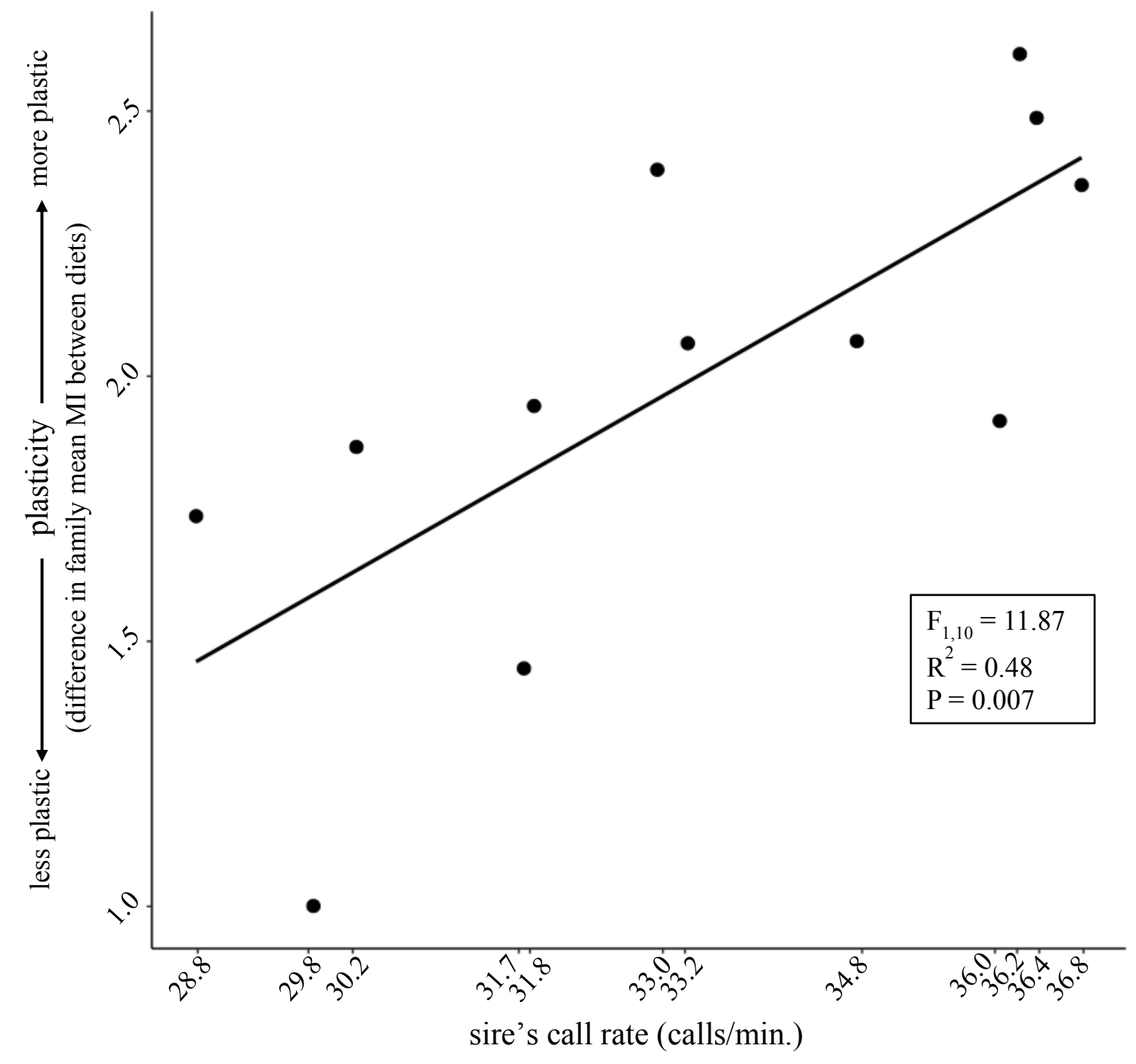

Figure 3.

Linear regression of plasticity in resource-use morphology (calculated as differences between familymean MI values on shrimp versus on detritus diets) on ln call rate (back-transformed). 


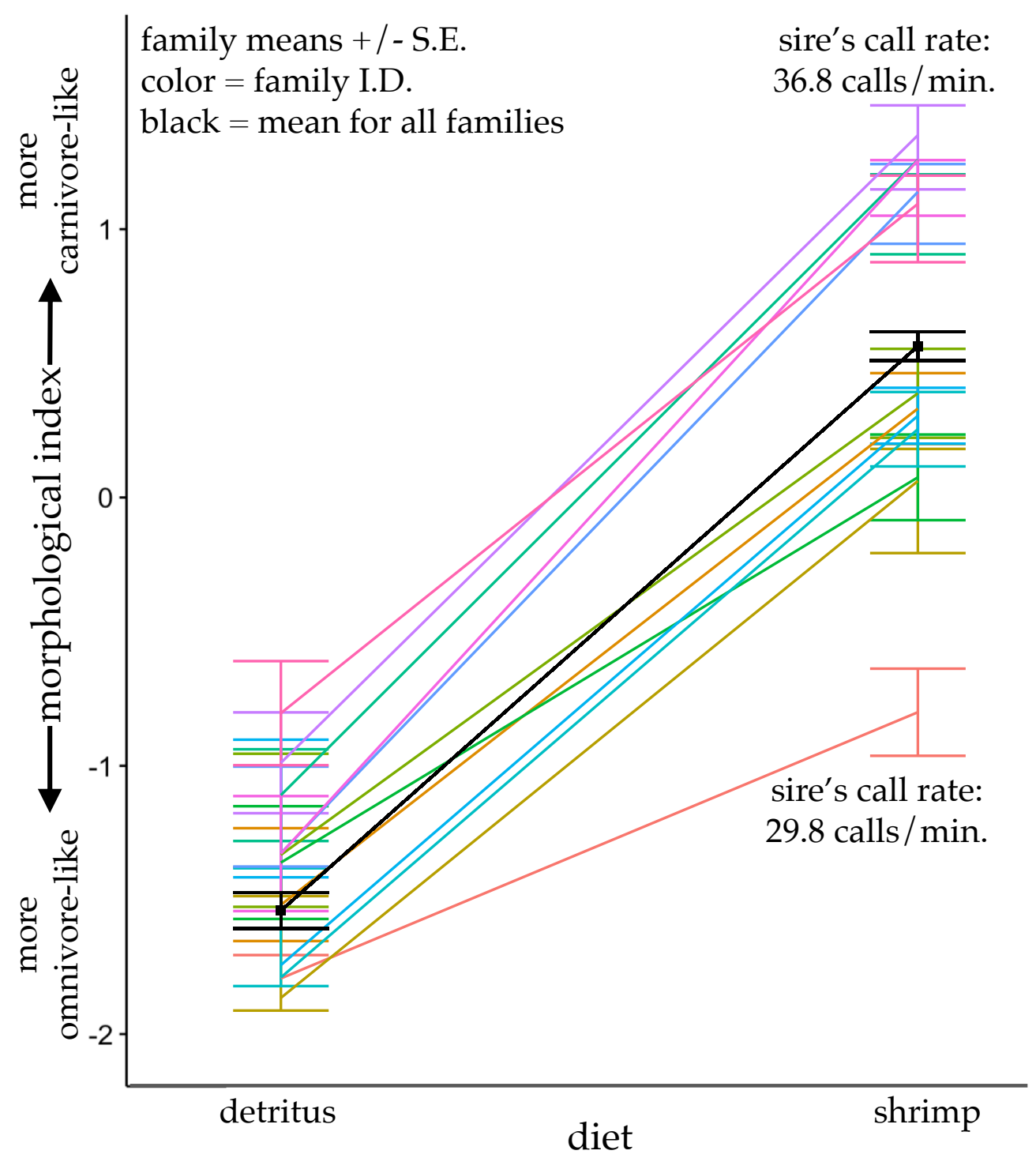

Figure 4.

Reaction norms of family mean morphological index values when tadpoles were reared on detritus versus shrimp diets. Call rates are shown for sires whose tadpoles exhibited the steepest and shallowest reaction norms. 


\section{Discussion}

We evaluated whether sexual selection and phenotypic plasticity interact to promote local adaptation in variable environments. In particular, we sought to determine whether females could use male sexual signals as an indicator of adaptive offspring plasticity. As we highlight below, female mate choice for males that sire plastic offspring could both promote local adaptation to variable or changing environments and favor the evolution and maintenance of plasticity.

Using spadefoot toads as our study system, we found that a male sexual trait (male call rate) predicted the expression of environmentally induced resource-use morphology (i.e., a distinctive carnivore ecomorph) in the male's offspring (Fig. 2). We also found that male call rate predicted the degree of trait integration in his offspring as well as the degree of plasticity in the expression of his offspring's resource-use morphology (Figs. 3 and 4). Previous work, focusing on the same populations studied here, shows that females prefer faster-calling males [167, 169]. By doing so, females select males that are generally in better condition and that sire more and larger offspring $[167,168]$. Our results reveal an additional benefit of choosing faster-calling males: the production of offspring capable of adopting the resource-use morphology best suited for prevailing conditions. In this case, not only did faster-calling males sire more carnivore-like offspring on a shrimp diet (i.e., their offspring produced the resource-use morphology that can best utilize the shrimp resource [180]), they also produced offspring that displayed greater plasticity in resource-use morphology when reared on different diets (shrimp versus detritus). Plasticity could therefore be an indirect 'good genes' benefit that enhances the fitness of a females' offspring (and, indirectly, the female). 
The carnivore and omnivore resource-use phenotypes of spadefoots carry different fitness costs and benefits. The nutrient-rich shrimp and tadpole diet of the carnivore phenotype fuels rapid development and, in many cases, an overall larger body size at metamorphosis [3]. Yet, carnivores can face intense competition for resources, especially when prey densities are low and the number (or quality) of competitors is high [173]. Omnivores, by contrast, have lower chances of survival (owing to their slower development and smaller size at metamorphosis), but they face less resource competition and can actually metamorphose in better overall body condition if they have sufficient time to develop [3].

As a consequence of these different costs and benefits, the optimal resource-use morphology that a given tadpole should adopt varies depending on the number of competitor carnivores, the resources available (i.e., the relative abundance of carnivore versus omnivore resources), and the size of the pond (i.e., whether it will dry quickly or more slowly). Each of these factors, in turn, varies both spatially (different ponds can hold more/less water or resources or have different competitor densities) and temporally (rainfall varies year-to-year, as does resource density, which can be tied to pond size) $[3,166]$.

Such a variable environment is expected to favor the evolution of phenotypic plasticity. Indeed, it has long been assumed that plasticity will be favored when: organisms confront environmental variation; no fixed trait is best suited for all environmental conditions; cues are available that reliably signal change in local conditions; and the fitness benefits outweigh the costs of expressing plasticity [39, 40, 81]. These conditions apply to spadefoot tadpoles in the variable pond environments described above: neither the omnivore nor the carnivore phenotype is best suited for all conditions [3]; shrimp density and water depth reliably signal resources, competition, and pond longevity [27]; and the plasticity in 
resource-use morphology appears to bear relatively few costs compared to the benefits [188]. Thus, plasticity is a trait that is expected to be favored in this system, and females that prefer males who sire more plastic offspring would likely benefit (by producing more successful offspring) compared to females that lack such preferences. Consequently, female mate choice would promote the evolution of plasticity by generating an added advantage to males who sire more plastic offspring — namely, enhanced mating success.

The possibility that sexual selection—specifically, female choice—might promote the evolution of phenotypic plasticity has not generally been considered. This is especially true for cross-generation or indirect ('good genes') effects, whereby females benefit from the production of plastic offspring (Fig. 1b). Generally, because plastic offspring should have higher fitness than non-plastic offspring in rapidly changing or highly variable environments, females should benefit by preferring males that sire plastic offspring in such environments (Fig. 1a). Yet, because females are unlikely to be able to directly assess a male's ability to sire plastic offspring, females might generally have to rely instead on male sexual signals that reliably indicate the plasticity of his offspring. If male sexual signals do indeed indicate a male's ability to produce plastic offspring (as we have shown here for spadefoots), and if females prefer such males (as has previously been shown for spadefoots $[167,169]$ ), then sexual selection could thereby favor the origin and maintenance of plasticity. Indeed, given the ubiquity of female choice in sexually reproducing species $[6,8]$, female mate choice might play an important and general role in favoring and/or reinforcing the evolution of phenotypic plasticity in many species. Future research on diverse species is needed to ascertain the frequency with which plasticity constitutes a fitness benefit of mate choice, and to clarify female choice's role in the evolution of plasticity. 
Conversely, female choice could also explain the evolution of decreased plasticity and even the complete loss of plasticity. The evolutionary loss of plasticity_-'genetic assimilation' (sensu [189]) — is increasingly viewed as playing a potentially important role in the origins of novel traits and even new species [144, 145]. However, researchers have long struggled to explain why selection would ever favor the loss of plasticity [190], unless that plasticity is costly [191]. Yet, few studies have identified such costs of plasticity [192], which deepens the paradox as to why selection would favor the complete loss of plasticity [190]. Our results provide a possible resolution to this paradox: sexual selection as mediated by female mate choice might, under certain circumstances, favor the loss of plasticity. For example, females might be under selection to prefer a more restricted range of male traits (for instance, if they encounter a closely related heterospecific that uses similar sexual signals, as has occurred in certain populations of our focal species [167]), which could simultaneously lead to female choice of males that produce non-plastic offspring. Female choice could thereby promote the loss of plasticity. Likewise, if the benefits of offspring plasticity were concomitantly reduced —-for example, if a particular inducible phenotype experienced lower relative fitness, as has also occurred in some populations of our focal system $[26,38]-$ then females would be under selection to avoid the production of plastic offspring and might evolve preferences for males who produced less (as opposed to more) plastic offspring. In this way, mate choice could also promote an evolutionary loss of plasticity. Either way, sexual selection might be an underappreciated force favoring genetic assimilation. Further studies are needed to address the role of female choice in genetic assimilation.

Sexual selection's interaction with plasticity is not limited to 'good genes' effects in the offspring of males and females. As highlighted in Table 1 and the Introduction, plasticity 
could evolve in the context of male signalling or the expression of female mate choice. If, for example, adults can assess their offspring's environment reliably, selection could favor males that produce different signals of quality in different environments $[159,193,194]$.

Additionally, selection could favor females that facultatively modify their mate preferences so as to optimize their fitness and that of their offspring across different environments (Table $1 ;[8,164,165])$. Whether selection favors adaptive plasticity at the adult stage (during signalling or mate choice) versus the offspring stage (via 'good genes' effects as seen in this study) will likely depend on the conditions above, and whether adults (as opposed to offspring) are better positioned to assess the cues associated with the production of fitness enhancing traits in a given environment. Although a growing body of work has revealed how plasticity interacts with sexual selection in generating condition-dependent signals and preferences in adults [8], additional work is needed to evaluate when parents - as opposed to their offspring — will evolve plasticity.

Beyond contributing to understanding of how sexual selection and plasticity impact the evolution, our results also provide insight into the problem of whether sexual selection facilitates or inhibits local adaptation $[72,157,159,195,196]$. Sexual selection should inhibit local adaptation if patterns of mate choice and male signalling generate a mating advantage for males who sire offspring that are disfavored in the given environment by natural selection (Fig. 1a). Sexual selection's inhibitory effects on local adaptation are expected to be greatest in variable environments if sexual selection generates evolutionary time lags whereby mate choice and male signals fail to track environmental change (Fig. 1a). Previous work has failed to fully resolve the problem of whether sexual selection can facilitate local adaptation in variable or changing environments $[159,196]$. This study 
suggests that adaptive offspring plasticity represents a solution to this problem. If females preferentially choose males that sire plastic offspring capable of adopting phenotypes best suited for their prevailing environment (Fig. 1b), then sexual selection could facilitate local adaptation even in the most variable and rapidly changing environments. 
Table 1. Alternative Routes whereby Sexual Selection can Impact the Evolution of Phenotypic Plasticity.

\begin{tabular}{|c|c|}
\hline Route & Description of route \\
\hline $\begin{array}{l}\text { Plasticity in } \\
\text { secondary sexual } \\
\text { traits }\end{array}$ & $\begin{array}{l}\text { 1. Competitively mediated, disruptive, intra-sexual selection can } \\
\text { favor the evolution of alternative condition-dependent (plastic) } \\
\text { secondary sexual traits (e.g., ornaments, signals, weapons) [82, } \\
\text { 197, 198]. } \\
\text { 2. Variation in the environment can favor plastic expression of } \\
\text { sexual signals that: a) are more readily detected by females in that } \\
\text { environment; or b) better indicate male quality (ability to confer } \\
\text { fitness benefits to females or their offspring) in the given } \\
\text { environment [194]. }\end{array}$ \\
\hline $\begin{array}{l}\text { Plasticity in mate } \\
\text { choice }\end{array}$ & $\begin{array}{l}\text { 1. If the fitness consequences of mate choice vary depending on } \\
\text { circumstances, selection will favor condition- or context- } \\
\text { dependent female preferences or choosiness. Indeed, in many } \\
\text { species, female choice is sensitive to variation in factors such as } \\
\text { parasites, predators, season, and the female's diet, social status, } \\
\text { and age [8, 165]. } \\
\text { 2. In variable environments (or where choice is costly), selection can } \\
\text { favor females that copy the mate preferences of other females } \\
\text { (e.g., older or more successful females) or that learn preferences } \\
\text { based on environmental exposure [8]. }\end{array}$ \\
\hline $\begin{array}{l}\text { Choice for mates } \\
\text { that sire plastic } \\
\text { offspring }\end{array}$ & $\begin{array}{l}\text { Female mate choice favors the evolution of plasticity in offspring when } \\
\text { they prefer males that sire plastic offspring [see this study]. }\end{array}$ \\
\hline
\end{tabular}




\section{CHAPTER 4: A CONDITION-DEPENDENT MALE SEXUAL SIGNAL PREDICTS ADAPTIVE PREDATOR-INDUCED PLASTICITY IN OFFSPRING}

The following is adapted from:

Kelly, P.W., Pfennig D.W., and Pfennig, K.S. 2021. A condition-dependent male sexual signal predicts adaptive predator-induced plasticity in offspring. Behavioral Ecology \& Sociobiology. 75: 28.

\section{Introduction}

Can sexual selection promote adaptation, especially in variable environments? Theory predicts that mate preferences for exaggerated, condition-dependent sexual signals can promote adaptation by generating adaptive associations between sexual traits (mate preferences and sexual signals) and offspring fitness [11-15, 32, 33]. Yet other theory conversely predicts that environmental variation can generate mismatches between adult sexual traits and offspring phenotypes and fitness, which can preclude sexual selection from promoting adaptation in variable environments [16, 18-21]. However, if sexual signals predict adaptive offspring plasticity, then mate preferences for such signals will favor the production of offspring that can express adaptive phenotypes under prevailing environmental conditions, even if those conditions differ from those experienced by the parents. In other words, adaptive phenotypic plasticity in offspring could constitute a fitness benefit of mate choice, and sexual selection via mate choice for plastic offspring could thereby promote adaptation to variable environments [4]. 
The hypothesis that offspring plasticity could function as a fitness benefit of mate choice rests on conventional predictions of mate choice theory [sensu 6]. In particular, because direct assessment of potential mates' capacity to produce high-quality offspring is unlikely [6], sexual signals should reliably indicate potential mates' capacity to produce adaptively plastic offspring. Further, for such signals to remain honest indicators of the ability to produce plastic offspring, they should be condition-dependent or otherwise costly to produce $[23,31]$. Yet, despite the potential for sexual signals to indicate whether a prospective mate can produce adaptively plastic offspring, few studies have examined this possibility.

We therefore sought to determine whether a form of adaptive tadpole plasticity that is widespread among amphibians is predicted by male sexual signals in spadefoot toads, Spea multiplicata. Specifically, we tested whether male sexual signals predict the expression of tadpole tail-fin plasticity in response to cues that signal the possible presence of predators. This type of tadpole plasticity occurs among diverse amphibian taxa [e.g., 199, 200-205], and experiments using multiple taxa have demonstrated that such plasticity is adaptive [e.g., 121, 123, 206, 207, 208]. In particular, tadpoles develop deeper tails in response to predator cues, which facilitates escape; however, deeper tails are associated with slower growth and so are disfavored in the absence of predators [121, 123, 206-209]. We found that S. multiplicata tadpoles developed deeper tail fins in response to predation cues and that this plasticity is associated with both male (sire) body condition (size-adjusted mass) and male sexual signals (calling effort). Thus, offspring plasticity might function as an indirect benefit of mate choice and allow sexual selection to facilitate adaptation in variable environments. 


\section{Material and Methods}

\section{(a) Study System}

Spea multiplicata occur in dry areas of southwestern North America [171, 210]. They typically breed once annually in ephemeral, rain-filled ponds $[3,27,166]$. Males call to attract females, and females choose their mates on the basis of their call characteristics, primarily call rate (calls per minute) $[28,171]$. In most populations, females prefer relatively high-condition males that produce relatively rapid call rates, which are associated, in turn, with higher fertilization success and enhanced offspring growth [28, 29]. Females choose their mates by closely approaching and touching a male, which initiates pair formation [28].

Spea tadpoles exhibit resource-use polyphenism: they can develop a default “omnivore" phenotype with generalized trophic morphology or, as a result of intense intraspecific competition and ingestion of live prey, develop an inducible "carnivore" phenotype with trophic morphology specialized for catching and consuming live prey $[3,27$, 170]. This polyphenism is a product of negative frequency-dependent selection $[3,25,82]$. Importantly, equilibrium frequencies of carnivores vary within and across generations [3, 170, 211-213]. This variable frequency-dependent selection therefore favors the capacity for tadpoles to assess their environment and respond accurately by developing either the omnivore or carnivore phenotype via plasticity [3, 27, 170, 211-213].

In this study, we chose to evaluate the capacity for omnivore tadpoles to express tailfin plasticity. We did not use carnivore tadpoles for the following reasons. Tadpoles that express the carnivore phenotype feed on Anostracan fairy shrimp and other tadpoles, including conspecifics $[27,170,214]$. Carnivore tadpoles are likely the primary source of predation on omnivore tadpoles [214], although predation by other predators, such as 
Odonate naiads, occurs as well [215]. Thus, both the sources and intensity of predation on omnivore tadpoles are variable, which should favor omnivore tail-fin plasticity. Further, if omnivore S. multiplicata tadpoles express tail-fin plasticity in response to predation on conspecifics, a likely inducing cue is the presence of injured conspecifics [i.e., an 'alarm cue' sensu 216], rather than a predator-specific cue. Thus, we used injured-conspecific cues as a proxy for predation in our experiment (see below).

\section{(b) Field Collections and Tadpole Rearing}

At a natural breeding aggregation on the night of 01 August 2019 at approximately 22:00 hours in southeastern Arizona, USA (315' $10.8^{\prime \prime} \mathrm{N}, 109^{\circ} 09^{\prime} 40.2^{\prime}$ W), we individually recorded 10 calling $S$. multiplicata males for approximately one minute and then collected them. The water temperature at the time of recording was $16.25^{\circ} \mathrm{C}$. Males at the breeding aggregation had been calling for at least two hours before we began recording. Oneminute recordings are sufficient to capture representative variation in this continuously calling species: $S$. multiplicata males produce 31 calls per minute on average, and the duration of each call is 1.1 seconds on average [28], and comparisons with longer recordings show no effect of recording length on measures of male call parameters (G. Calabrese and KSP, unpublished data). Moreover, in Arizona, S. multiplicata breed on a single night in a given population, with both male calling and female mate choice occurring in the span of several hours; therefore, variation in which males attend a given aggregation (i.e., the male competitive environment) and night-to-night variation in temperature do not confound mate choice $[28,171]$. In addition, the 10 males that we recorded and collected represented more than half of all males calling at the breeding aggregation; as is common for S. multiplicata $[28,171]$, this aggregation consisted of fewer than 20 calling males, which were surveyed 
without difficulty in the small pond in which the aggregation formed. We measured all males for snout-to-vent length (SVL) and mass. We also collected 10 female $S$. multiplicata at the same aggregation.

Immediately following collection, we randomly paired each recorded male with a female for breeding in separate tanks filled with $6 \mathrm{~L}$ of dechlorinated well water at the Southwestern Research Station. After approximately 8h, upon visual confirmation that oviposition had occurred, we removed the adults from the tanks and began aerating the eggs. Approximately $48 \mathrm{~h}$ later, after all tadpoles had hatched and begun swimming, we provided each tank with 20mg of crushed detritus (TetraFin® Fishfood Pellets), which mimics the natural diet of omnivore tadpoles and does not induce expression of the carnivore phenotype [183]. We continued daily feedings of 20mg detritus per tank for three days and then increased to $40 \mathrm{mg}$ daily for the subsequent three days. On the sixth day after tadpoles hatched, we transported the tadpole sibships via automobile in their respective rearing tanks to UNC Chapel Hill, which took three days during which we provided $40 \mathrm{mg}$ of detritus per tank twice daily. We did not quantify mortality during transport, but very few tadpoles died during transport, and we did not observe differences among the sibships in mortality. We also transported the adult $S$. multiplicata used in the breedings to UNC for addition to the colony housed there.

\section{(c) Experimental Methods}

At UNC, we set up six replicate microcosms $(34 \times 20 \times 12 \mathrm{~cm}$ plastic boxes with $6 \mathrm{~L}$ dechlorinated tap water) per sibship. We designated three microcosms per sibship as controls, and the remaining three microcosms for each sibship comprised our treatment group. Each microcosm contained 10 visually size-matched siblings, such that each sibship had 30 
tadpoles per treatment (starting total $\mathrm{N}=600$ tadpoles). Visual inspection unambiguously confirmed that no tadpoles expressed the carnivore phenotype. On days one through nine of the experiment, we provided each microcosm 100mg detritus daily, and on days ten through twelve, we provided each microcosm $120 \mathrm{mg}$ detritus daily. We kept the remainder of each sibship in their original rearing tanks for use as stimuli (see below). We provided each original rearing tank $120 \mathrm{mg}$ detritus daily for the duration of the experiment.

On days two though twelve, we applied our injured-conspecific cue to the treatment group. To do so, we selected six tadpoles from each sibship's original rearing tank and euthanized them in a $0.1 \%$ aqueous solution of tricaine methanesulfate (MS-222). Then, we crushed them, thoroughly rinsed them with dechlorinated water to remove MS-222, and used a dipnet to place two per microcosm in each of the corresponding sibship's three treatment microcosms. On days three through twelve, we removed the previous day's crushed tadpoles from each treatment microcosm before adding fresh ones (there was very little consumption of the crushed tadpoles, and growth did not differ between control and treatment groups; see Results). In addition, on days two through twelve, we disturbed the water in the control microcosms with a clean, empty dipnet in a manner that simulated placing crushed tadpoles in the treatment microcosms.

On day 13, we euthanized all experimental tadpoles in MS-222 (we also euthanized all remaining tadpoles in the original rearing tanks). Immediately after euthanizing them, we photographed the tadpoles from a lateral point of view using a Canon digital SLR camera and $50 \mathrm{~mm}$ macro lens. After photography, we stored the euthanized tadpoles in $95 \%$ ethanol. Owing to mortality, our final sample size was 496 tadpoles (240 in the control group and 256 in the cue group). 
To minimize observer bias, we had a trained observer perform all tadpole measurements without knowledge of the experimental protocol or microcosm designations. The observer used ImageJ to measure SVL and maximum tail depth (ventral maximum of ventral fin to dorsal maximum of dorsal fin) for each tadpole.

\section{(d) Call Analyses}

We used the computer program Audacity to calculate two condition-dependent temporal characteristics of male calls. The first, call rate, has previously been shown to predict tadpole phenotypes and fitness $[4,28,29]$. The second, call effort, is commonly used as a proxy for "vocal performance," or the energetic expenditure made by male frogs while calling $[217,218]$. We calculated call rate as calls per minute, and we calculated call effort as the product of call rate multiplied by call duration (i.e., mean call length in seconds) [217, 218]. To minimize observer bias, we used blinded methods when performing call analyses.

\section{(e) Statistical Analyses}

We performed all analyses using R [R Core 219]. To account for tadpole tail depth allometry, we used residuals from a linear regression of $\ln$ tadpole tail depth on $\ln$ tadpole SVL in place of raw tail depth measurements.

To determine whether S. multiplicata tadpoles developed deeper tail fins in response to the injured-conspecific cue, we used a linear mixed-effects model in the $\mathrm{R}$ package lmerTest [220]. Our response variable was SVL-corrected tail depth, and our fixed effects were treatment (control or cue) and sibship. To test for a genotype-by-environment (GxE) effect, we also included the interaction of treatment and sibship as a fixed effect. We specified replicate as a random effect, and we used an F-test with Kenward-Roger approximations to degrees of freedom for inference. In addition, to assess whether growth 
differed between treatments, we used a linear mixed-effects model with SVL as the response variable and treatment as the fixed effect. For random effects, we specified sibship and replicate. We again used an F-test with Kenward-Roger approximations to degrees of freedom for inference.

To determine whether sire condition and/or call characteristics predicted the expression of tail-fin plasticity, we calculated a plasticity index for each sibship. We calculated this index as Hedge's g effect sizes for each sibship's response to the experimental treatment. Hedge's g consists of the difference in treatment means (each sibship's mean SVL- corrected tail depth in the control versus cue treatments) divided by the pooled weighted standard deviation (the square root of the sum of the squared standard deviations from each treatment divided by two). Hedge's g is very similar to Cohen's d but performs better with small sample sizes [221]. Next, we calculated male (sire) body condition as the scaled mass index (SMI), which adjusts mass for a given SVL via standardized-major-axis regression [222]. Then, we regressed the plasticity index on sire call rate, call duration, call effort, and condition (in separate regressions). In addition, to test for condition dependence of call characteristics, we regressed them on male condition.

Finally, we used logistic regression in a generalized linear mixed-effects model to determine whether mortality differed among cue and control groups or among sibships. We first specified treatment, sibship, and the interaction of those terms as fixed effects and replicate as a random effect, but no models that included sibship converged. However, a model with sire call effort and treatment as fixed effects and replicate as a random effect converged, and we report that model below. We additionally report models with sire call rate, 
call duration, and body condition (SMI) substituted for call effort (in separate models), with all other model terms unchanged. We used Wald chi-squared tests for inference.

\section{Results}

Tadpoles developed deeper tail fins in the treatment group than in the control group $\left(F_{130.927}=8.334, P=0.007\right.$; Fig. 1$)$. This effect was independent of body size: we corrected tail depth for SVL, and we did not detect an effect of treatment on SVL in a mixed model $\left(F_{1,477}=\right.$ $1.701, P=0.226$ ). Thus, $S$. multiplicata exhibit plastic responses to predation (in the form of injured-conspecific cues) like those of other amphibian taxa. In addition, we found GxE effects on tail depth (treatment-by-sibship interaction: $F_{1,38.72}=7.412, P=0.010$; Fig. 2).

Male body condition (SMI) predicted the expression of tadpole tail-fin plasticity $\left(F_{1,8}\right.$ $=17.460, \mathrm{R}^{2}=0.65, P=0.003 ;$ Fig. 3a). In particular, males with relatively high body condition sired tadpoles that exhibited the greatest tail-fin plasticity.

Call rate did not predict the expression of tadpole tail-fin plasticity $\left(F_{18}=0.036, P=\right.$ $0.854)$, but call effort $\operatorname{did}\left(F_{18}=7.319, \mathrm{R}^{2}=0.41, P=0.027\right.$; Fig. $\left.3 b\right)$. Males that exhibited relatively high call effort sired tadpoles that exhibited the greatest tail-fin plasticity. This relationship appears to be driven primarily by call duration; males with longer call durations sired tadpoles with greater tail plasticity $\left(F_{1.8}=8.669, \mathrm{R}^{2}=0.46, P=0.019\right)$. Further, although condition did not predict call rate $\left(F_{1,8}=0.001, P=0.983\right)$, it did predict call effort $\left(F_{18}=\right.$ $\left.6.998, \mathrm{R}^{2}=0.40, P=0.030\right)$ and call duration $\left(F_{1,8}=7.232, \mathrm{R}^{2}=0.41, P=0.028\right)$, consistent with call effort being a condition-dependent call character.

We did not detect an effect of treatment on tadpole mortality $\left(\chi^{2}=0.441, \mathrm{DF}=1, P=\right.$ 0.507), but sire call effort predicted tadpole survival. Specifically, tadpoles sired by males 
with higher call effort were likelier to survive to the end of the experiment $\left(\chi^{2}=4.857, \mathrm{df}=1\right.$, $P=0.0275$ ). No other sire characteristics predicted survival (call rate: $\chi^{2}=1.251, \mathrm{df}=1, P=$ 0.263; call duration: $\chi^{2}=0.061, \mathrm{df}=1, P=0.805$; body condition $(\mathrm{SMI}): \chi^{2}=0.057, \mathrm{df}=1, P$ $=0.811)$. 


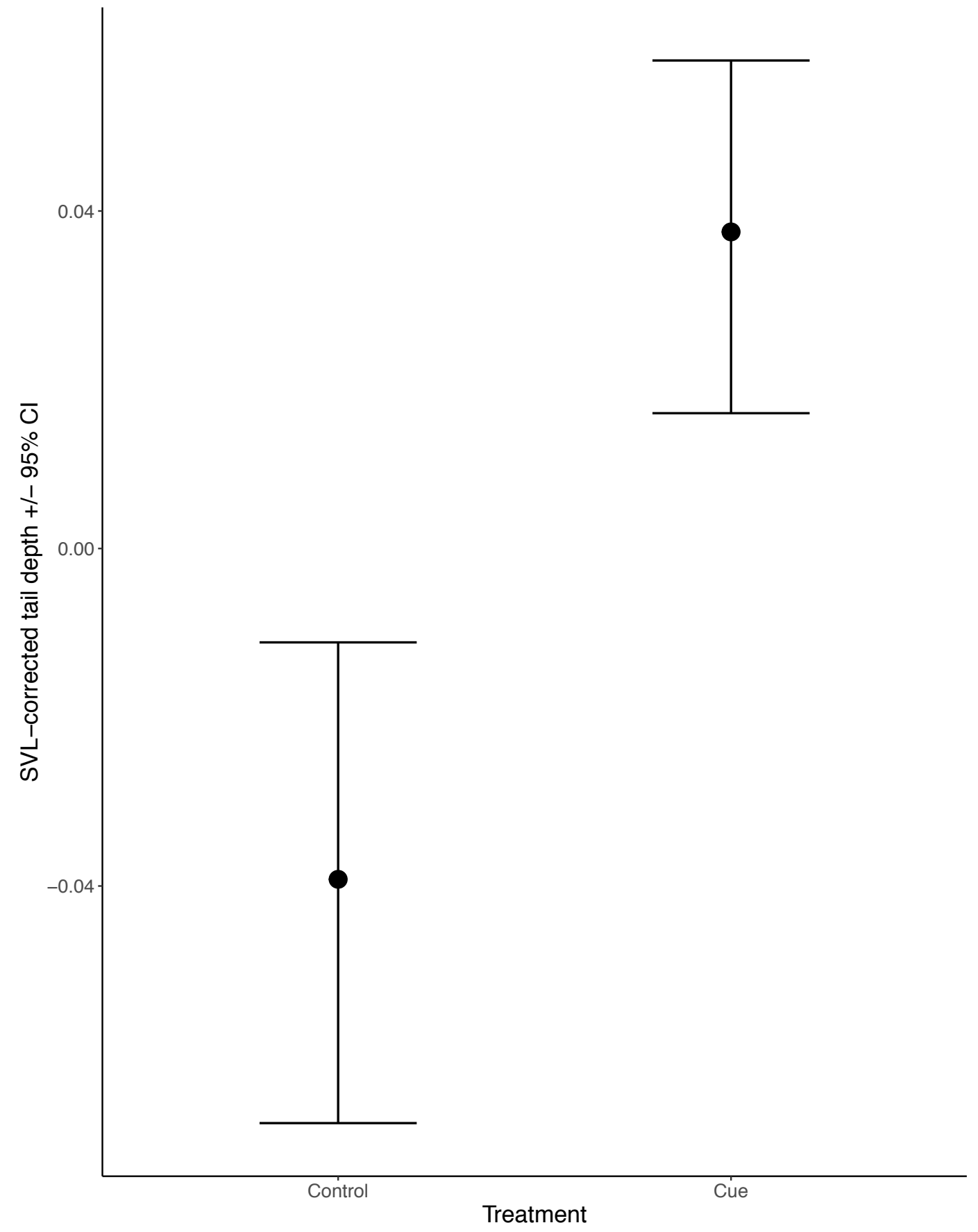

Fig. 1

Mean SVL-corrected tail depth with $95 \%$ bootstrapped CI for all tadpoles reared in the control and cue treatments. Bootstrapping was performed with 1,000 simulations in the 'boot' function of the R package boot [1]. SVL-corrected tail depth consists of residuals from the regression of $\ln$ tail depth on $\ln \mathrm{SVL}$ 


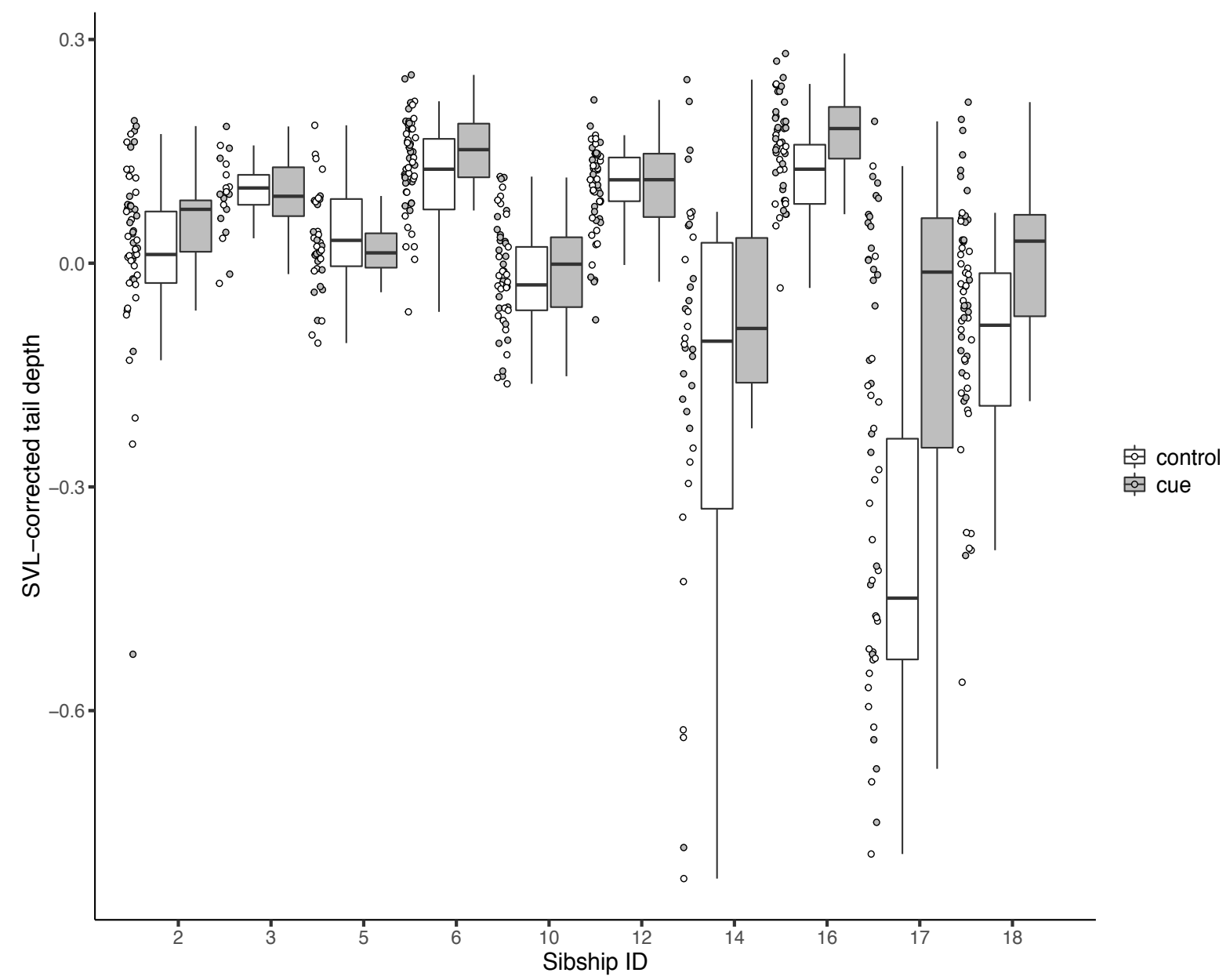

Fig. 2

SVL-corrected tail depths (points) and box-and-whisker plots for each sibship and each treatment. Points are immediately to the left of their corresponding box-and-whisker plots. The box-and-whisker plots are as follows: thick black lines represent the medians, boxes represent the interquartile ranges, and whiskers extend to the most extreme points within $1.5 x$ the interquartile ranges outside the boxes. SVL-corrected tail depth consists of residuals from the regression of $\ln$ tail depth on $\ln$ SVL. Sibships are displayed in numerical order of their identifying numbers from the experiment. Points are jittered slightly on the $\mathrm{x}$ axis. 


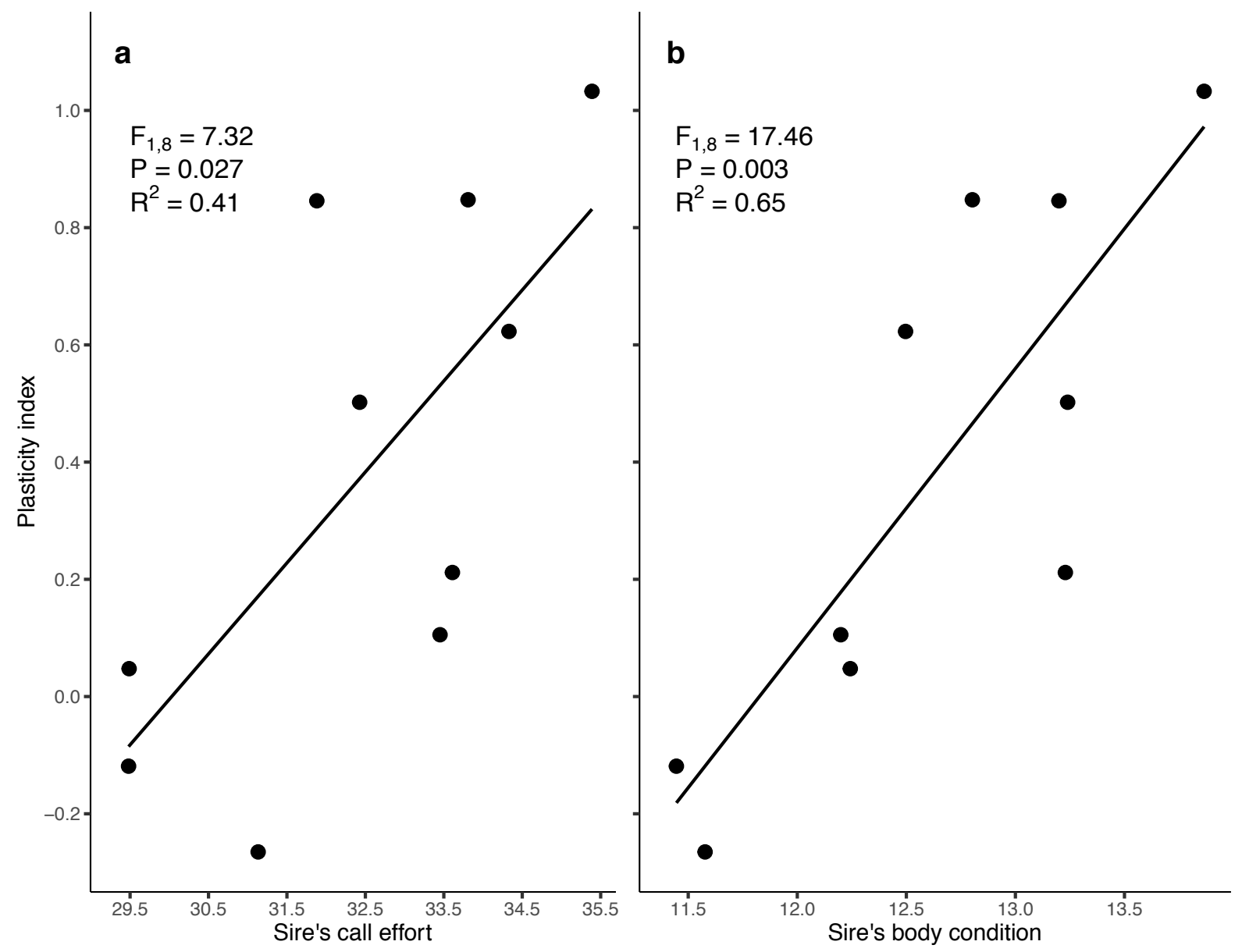

Fig. 3

a) Linear regression of tadpole plasticity on sire call effort (the product of call rate [calls $/ \mathrm{min}$ ] multiplied by call duration [sec]). Slope estimate $=0.156+/-0.058(1 \mathrm{SE})$.

b) Linear regression of tadpole plasticity on sire body condition (body condition $=$ Standardized Mass Index, which adjusts mass for a given SVL). The plasticity index consists of Hedge's g effect sizes; higher values indicate greater plasticity, i.e., greater difference between mean SVL-corrected tail depth in the cue treatment compared to control (see Methods for further details). Slope estimate $=0.477+/$ 0.114 (1 SE). 


\section{Discussion}

An association between adult sexual traits and offspring plasticity can maintain the relationship between sexual traits and offspring fitness that is required if sexual selection promotes adaptive evolution in variable environments. We investigated a key assumption of this hypothesis by evaluating whether male call characteristics predict the expression of a taxonomically widespread form of tadpole plasticity. We showed that tadpoles of our study species, Spea multiplicata, develop deeper tails in response to a proxy for predation: injuredconspecific cues (Fig. 1). Further, we found that both male body condition and call effort predicted the expression of tadpole tail-fin plasticity in response to injured-conspecific cues. Specifically, higher condition males with higher call effort sired tadpoles that exhibited greater plasticity in tail-fin depth (Fig. 3). Although some might contend that our sample size (10 sires) was small, the 10 males sampled represented the majority of males calling in the population that we sampled (see above). Furthermore, our results are consistent with published data showing that male $S$. multiplicata call characteristics predict adaptive plasticity in other tadpole traits [4]. Importantly, the present study details not merely a similar predictive relationship between call characteristics and tadpole plasticity, but one that involves a form of plasticity that is common among diverse amphibian taxa [223].

Indeed, the existence of similar forms of sexual signaling among anuran taxa [76] and the broad taxonomic distribution of this form of tadpole tail plasticity suggest that offspring plasticity might commonly maintain adaptive associations between sexual traits and offspring fitness in variable environments. In particular, the capacity to develop deeper tail fins in response to injured-conspecific or predator cues has been demonstrated to be adaptive in the context of predator avoidance in multiple anuran taxa [e.g., 121, 123, 206, 207, 208]. Further, 
although we did not measure tadpole fitness as a function of tail plasticity, the cue that we used in our experiment is likely a reliable indicator of predation risk in this system [214, 215]. Therefore, the plasticity demonstrated here is likely adaptive.

That the adaptive offspring plasticity is associated with male call effort and body condition suggests that it could function as an indirect fitness benefit of mate choice.

Previous work has found that females prefer faster calling males but show no discrimination between male calls that differ in duration [28]. In these preference tests, only one call feature was manipulated at a time; all other call characters were controlled. However, females have not been tested for their preferences of call effort per se, and preferences for call rate and call duration might interact in ways that have not been captured in previous studies. Thus, whether females use call effort to choose mates who sire offspring with greater tail plasticity is an open question. However, we additionally found that male call effort predicted tadpole survival in this experiment, which suggests that females may benefit from choosing to mate with high-call-effort males nonetheless.

Interestingly, although male call rate did not predict tadpole tail-fin plasticity, call rate does predict another form of tadpole plasticity; namely the likelihood of expressing the carnivore morph [4]. Different components of frog calls are often indicators of different phenotypes, such as species identity, fecundity, body condition, or offspring characteristics [76]. Moreover, female frogs are known to assess multiple call components [28, 76, 124, 125]. It is therefore plausible that different components of $S$. multiplicata calls communicate different offspring characteristics, including different forms of plasticity. This possibility requires further research. 
Our finding that both male call effort and body condition predict the expression of tadpole tail-fin plasticity further suggests the possibility that mate preferences for conditiondependent signals can facilitate adaptation in variable environments. Generally, because plasticity is likely to mediate offspring fitness in variable environments [40], only those offspring capable of facultatively expressing adaptive phenotypes in a given environment are likely to achieve the condition necessary to producing preferred sexual signals [sensu 33, 34, 35]. This type of condition dependence is central to theoretical models that suggest that mate choice can promote adaptation because it couples nonsexual fitness with sexual traits $[13,15$, 33-35]. Our results suggest that offspring plasticity can mediate such associations and allow sexual selection to promote adaptation in variable environments. 


\section{CHAPTER 5: CONCLUDING REMARKS}

To conclude, I briefly return to the Outstanding Questions highlighted in Chapter 2. I think that each of these questions warrants greater discussion and suggestions for future research.

What are the genetic, developmental, and evolutionary mechanisms by which offspring plasticity becomes associated with adult sexual traits?

Addressing this question is fundamental to our understanding of whether and how plasticity serves as a fitness benefit of mate choice, not to mention our understanding of the mechanistic underpinnings of sexual traits and plasticity in general. There is a rich body of theory on the genetic, developmental, and evolutionary mechanisms underlying plasticity [see, e.g., 7, 190, 224, 225], though empirical approaches remain nascent [e.g., 187, 226, 227, 228], and the situation is similar regarding sexual traits in terms of theory [see, e.g., 8,13 , 23], though empirical inquiry is more advanced [reviewed in 8]. What is perhaps most interesting - and most informative in the context of adaptive offspring plasticity functioning as a fitness benefit of mate choice - is the degree of similarity among theoretical expectations and empirical findings regarding the mechanistic bases of both sexual traits and plasticity.

Indeed, plasticity has long been a central element of the study of sexual selection and mate choice, especially with regard to behavioral plasticity and the context- and conditiondependence of mate preferences and sexual signals $[6,8,11,229]$. Importantly, condition dependence may represent one of the most promising potential solutions to the question of what genetic and developmental mechanisms underlie associations between adult sexual 
traits and offspring plasticity. As discussed in Chapter 2, condition dependence is itself a special case of plasticity, and like other forms of plasticity, it usually has a heritable basis. Further, adaptive plasticity in nonsexual traits that is expressed early in life might readily contribute to heightened condition in adulthood by, for example, enabling greater acquisition of trophic resources, and thus contribute to the development of exaggerated, conditiondependent sexual signals or particular mate preferences [230]. Moreover, the heightened condition dependence of sexual traits in taxa ranging from arthropods to ungulates appears to share highly conserved developmental pathways, such as the insulin-like signaling pathway $[231,232]$, and similar physiological mechanisms have been implicated in polyphenism, including the spadefoot tadpole carnivore-omnivore polyphenism [225, 227].

Whether such associations are limited to condition dependence and plasticity in resource-use traits remains to be seen, but there are additional shared attributes that suggest they are not limited in this way. For example (and purely speculatively), the existence of two forms of adaptive larval plasticity (trophic resource-use phenotypes and predator-induced development of large tail fins) that are associated with a condition-dependent male sexual signal in spadefoot toads $[4,5]$ suggests that a common set of mechanisms might underpin such associations. Indeed, it may be that the expression of both adaptive offspring plasticity and condition-dependent sexual traits depends in many cases on the overall physiological state of an animal at different times in the course of its development.

This possibility points to a key question for future research on adaptive plasticity as a fitness benefit of mate choice: among taxa that express adaptive plasticity in various forms, such as spadefoot toad tadpoles, are particular genotypes simply better able to express each form of plasticity; does the expression of different forms of plasticity proceed separately; or 
are there tradeoffs in the expression of different forms of plasticity? There are examples of complementarity [e.g., 233], modularity [sensu 7], tradeoffs [e.g., 121], and other types of relationships among different forms of plasticity in various taxa [7, 234]. It may be that only certain types of such relationships are likeliest to become associated with adult sexual traits via a common mechanism like condition dependence. Assessing these possibilities by, for example, investigating the potential for correlated expression of different forms of offspring plasticity that are (and those that are not) associated with adult sexual traits, is likely a promising avenue for future research.

\section{Are there particular traits, taxa or conditions in which sexual traits are more likely to predict offspring plasticity?}

Evaluating these factors would provide insights into the processes by which plasticity serves as a fitness benefit of mate choice, although this may be the most difficult of these questions to answer, because it touches on key elements of each of the other three questions in this chapter (and Chapter 2). Table 1 of Chapter 2 offers a broad overview of the conditions expected to favor associations between offspring plasticity and adult sexual traits, and Box 1 of Chapter 2 highlights animals with complex life histories that are likely wellsuited taxa for study. In addition, Table 2 of Chapter 2 highlights specific, diverse taxa in which at least some of the conditions likely to favor associations between adult sexual traits and offspring plasticity are met.

The combination of those components of Chapter 2 illustrate the difficulty of answering whether there are particular traits, taxa, or conditions in which sexual traits are more likely to predict offspring plasticity. Beyond the occurrence of sexual selection (especially in the form of mate choice) and adaptive offspring plasticity, there are currently 
not clear circumstances in which to expect to find associations between offspring plasticity and adult sexual traits. Indeed, even those circumstances described in Chapter 2 could conceivably apply to a considerable range of traits, taxa, and conditions. This range of possibilities is certainly not a downside, however; rather, it may highlight a potentially ubiquitous dynamic in nature.

I expect that which traits, taxa, and conditions are likeliest to become involved in associations between adults sexual traits and offspring plasticity will likely hinge largely on the genetic, developmental, and evolutionary mechanisms that underlie those associations. If, as I speculated above, a common mechanism like condition dependence lies at the core of such associations, then I would expect them to be very common in nature. Yet it is also possible that a diverse collection of complex mechanisms are at work among different traits, taxa, and conditions (which is not meant to imply that condition dependence is at all simple [see, e.g., 231, 232]). As our understanding of these mechanisms, of indirect benefits of mate choice, and of the evolution of plasticity continues to grow, we will, I expect, begin to find additional intriguing examples of offspring plasticity functioning as an indirect benefit of mate choice.

\section{Can mate choice for offspring plasticity explain some of the conflicting evidence for indirect benefits of mate choice?}

If offspring plasticity commonly mediates connections between sexual traits and offspring fitness, then past tests of indirect benefits might have failed to capture important dimensions of how these models apply to natural populations (e.g., by rearing offspring in only one environment). Indeed, indirect benefits can be (and perhaps usually are) highly dependent on environmental context, with interactions among genotypes, phenotypes, and 
the environment determining whether correlations between adult sexual traits and offspring nonsexual traits do or do not amount to fitness benefits [6, 8, 21, 23, 235]. When offspring plasticity allows the development of adaptive phenotypes in a range of environmental conditions, however, and when it becomes correlated with adult sexual traits, as in spadefoot toads $[4,5]$, then environmental context need not obviate potential fitness benefits of correlations between sexual traits and offspring traits, which may be the case in other taxa [see, e.g., 235, 236].

In order to maintain such covariance between sexual traits and offspring fitness, indicator traits must be honest signals of potential mates' capacity to sire high-fitness offspring [31]. As discussed in Chapter 2, this criterion is generally expected to be met in cases of condition-dependent sexual signals $[6,8,10,23,31,32]$. In such cases, only those individuals capable of allocating considerable resources to signaling can express the forms of sexual signals that are favored by mate choice, and those individuals' offspring should inherit the potential to attain the same capacity in the course of development. Importantly, the capacity for offspring to express plastic responses to environmental cues in heterogeneous environments is likely to mediate offspring performance in those environments [39, 40, 81]. Therefore, offspring plasticity might readily couple offspring fitness with the capacity to express signals that are favored by mate choice in heterogeneous environments, because only those offspring capable of expressing adaptive developmental responses to their environment should attain the condition necessary to producing those signals. Further, and more broadly, because offspring plasticity is likely to mediate offspring performance in heterogeneous environments, then sexual signals that indicate males' capacity to sire adaptively plastic offspring should likewise function as signals of offspring fitness. 
This possibility does not necessarily require a purely genetic basis for offspring plasticity and its association with sexual traits (see Chapter 2), but it is entirely possible for a genetic basis to exist, which has important implications for good-genes models of sexual selection. First, it is important to note that good-genes benefits of mate choice consist of additive genetic effects on offspring fitness $[8,10,237]$ and that plastic traits commonly exhibit additive genetic variance in the heights and slopes of reaction norms $[42,49,151$, 238-240]. Such traits thus have a heritable basis of the type that can potentially amount to "good genes" if genotype-by-environment interactions among offspring covary with adult sexual traits. Such covariance may arise via genic capture [10,12]: additive genetic variance in adaptive offspring plasticity should contribute to variance in overall condition in the manner described above and thereby contribute to variance in condition-dependent sexual signals. Therefore, environmental heterogeneity and plastic responses thereto need not erode associations between adult sexual traits and offspring fitness as some have argued they should $[8,18]$. Rather, plasticity is commonly shaped by fluctuating selection in heterogeneous environments $[7,42,43,49,151,240]$ and likely mediates offspring fitness in such environments $[39,40,81]$, and its often-genetic underpinnings suggest that it has the potential to mediate associations between offspring viability and adult sexual traits - that is, to function as a good-genes benefit of mate choice. Thus, offspring plasticity is not simply environmentally induced stochasticity; rather, it is a trait with an evolutionary history that may be shaped by both natural and sexual selection.

Ideally, future investigations of indirect benefits will consider more than offspring viability (or fitness proxies). In addition to fitness measures, it will be important to understand offspring phenotypes - i.e., the targets of selection - and how those phenotypes 
arise in different environmental contexts (see Chapter 2). Rigorous criteria for establishing evidence of indirect benefits, especially good-genes benefits, have been thoroughly discussed elsewhere [e.g., 6, 15, 23, 241, 242], but to my knowledge, few studies have explicitly considered the potential role of plasticity in mediating the core element of good-genes benefits: indirect fitness benefits accrued to the choosing sex arising from the genetic contribution of the courting sex to offspring fitness [23; but see Chapters 3 and 4].

In addition, attempts to study indirect benefits seem to suffer from a bias in directionality: though many studies have investigated indirect benefits in the context of variation in adult sexual traits predicting variation in offspring fitness, considerably fewer have attempted the opposite and asked whether relatively high-fitness offspring are likelier to develop adaptive mate preferences and sexual signals [8, 18, 23, 242]. Doing so imposes several methodological difficulties, especially regarding the length of time required for a particular taxon to reach sexual maturity, which perhaps make it unsurprising that such studies are less common. For example, it remains unknown, in part due to methodological difficulties, if spadefoot toad tadpoles that are best able to express adaptive phenotypes in response to cues in their pond environment later develop, among females, preferences for fast-calling males or, among males, relatively fast call rates (see Chapters 3 and 4). Given what we have learned so far, I expect that this is likely the case, but formal tests will require considerable additional work. Still, establishing connections in both directions - adult sexual traits to offspring fitness and offspring fitness to adult sexual traits - would likely mark a major improvement for any body of research on indirect benefits [18, 23]. 


\section{Can sexual selection, paradoxically, lead to the loss of plasticity?}

Mate choice selecting indirectly on offspring plasticity could favor the evolutionary loss of plasticity and, consequently, thereby promote genetic assimilation. Genetic assimilation is a paradoxical process: it is known to occur and likely plays important roles in the origins of novel traits and in speciation, but explaining how selection would favor the complete loss of plasticity is difficult without invoking costs of plasticity, which are rarely detected and often very low when they do occur [46, 48, 59, 65, 190]. However, if offspring plasticity becomes associated with the sexual signals that can be assessed in the course of mate choice, then changes to mate preferences could favor those that produce less-plastic offspring, thereby compounding any costs of plasticity or of phenotypes that are produced via plasticity, and thus facilitate the process of genetic assimilation. Such changes to mating traits might not arise because offspring plasticity is selectively disfavored per se. Instead, selection might favor mating traits that maximize other components of fitness that are under stronger selection $[8,11,17,18,22,243-245]$. Thus, if selection for altered sexual traits coincides with selection against offspring phenotypes that arise from plasticity, then mate choice may facilitate genetic assimilation.

This may be a property of the spadefoot system described in preceding chapters. In Spea multiplicata, selection disfavoring ancestral female preferences might have facilitated a response to selection against plastic expression of the carnivore phenotype [181, 246]. Whether similar dynamics commonly arise requires additional research in this and other systems.

As highlighted in Chapter 2, there are numerous animal taxa, including spadefoot toads, in which mate choice occurs and in which offspring express (likely) adaptive plasticity 
in nonsexual traits (see Table 2 of Chapter 2). Any of those systems, and others like them, might prove valuable to assessing the potential role of mate choice in the genetic assimilation of previously plastic traits, especially if they are part of or include lineages among which the extent of plasticity exhibited by offspring varies [45, 225, 247]. The spadefoot toad example, though certainly not the only system in which the study of genetic assimilation is rapidly advancing, is illustrative: a derived lineage (certain populations of Spea bombifrons), that constitutively expresses a novel, formerly inducible larval resource-use phenotype can be compared, via competition experiments and other approaches, to highly plastic populations of Spea multiplicata, in which larval plasticity in resource-use traits likely functions as a fitness benefit of mate choice, as well as less-plastic, derived S. multiplicata populations that generally do not express the novel, inducible resource-use phenotype $[4,181,246]$. The combination of approaches to mate choice, sexual signals, and offspring plasticity described in Chapters 2-4 and references therein will, I hope, serve as an example for future research in diverse taxa intended to further our grasp of the potential role of mate choice in genetic assimilation. 


\section{REFERENCES}

1. Canty, A. and Ripley, B.D., boot: Bootstrap R (S-plus) Functions 2020.

2. Pfennig, K.S. and Rice, A.M. (2014) Reinforcement generates reproductive isolation between neighbouring conspecific populations of spadefoot toads. Proc. R. Soc. B 281 (1789), 20140949.

3. Pfennig, D.W. (1992) Polyphenism in spadefoot toads as a locally adjusted evolutionarily stable strategy. Evolution 46, 1408-1420.

4. Kelly, P.W. et al. (2019) Male sexual signal predicts phenotypic plasticity in offspring: implications for the evolution of plasticity and local adaptation. Phil. Trans. R. Soc. B 374 . 20180179 (1768).

5. Kelly, P.W. et al. (2021) A condition-dependent male sexual signal predicts adaptive predator-induced plasticity in offspring. Behavioral Ecology and Sociobiology 75 (2), 28. 6. Andersson, M. (1994) Sexual selection, Princeton University Press.

7. West-Eberhard, M.J. (2003) Developmental plasticity and evolution, Oxford University Press.

8. Rosenthal, G.G. (2017) Mate choice, Princeton University Press.

9. Pfennig, D.W. (2021) Key questions about phenotypic plasticity. In Phenotypic plasticity and evolution: causes, consequences, controversies (Pfennig, D.W. ed), CRC Press.

10. Rowe, L. and Houle, D. (1996) The lek paradox and the capture of genetic variance by condition dependent traits. Proc. R. Soc. B 263 (1375), 1415-1421.

11. Cotton, S. et al. (2006) Sexual selection and condition-dependent mate preferences. Curr. Biol. 16 (17), R755-65.

12. Lorch, P.D. et al. (2003) Condition-dependent sexual selection can accelerate adaptation. Evol. Ecol.Res. 5 (6), 867-881.

13. Veen, T. and Otto, S.P. (2015) Liking the good guys: amplifying local adaptation via the evolution of condition-dependent mate choice. J. Evol. Biol. 28 (10), 1804-15.

14. Proulx, S.R. (2002) Niche shifts and expansion due to sexual selection. Evol.Ecol.Res. 4 (3), 351-369.

15. Servedio, M.R. and Boughman, J.W. (2017) The role of sexual selection in local adaptation and speciation. Annu. Rev. Ecol. Evol. Syst. 48, 85-109. 
16. Bussiere, L.F. et al. (2008) Mate choice for genetic quality when environments vary: suggestions for empirical progress. Genetica 134 (1), 69-78.

17. Candolin, U. (2019) Mate choice in a changing world. Biol. Rev. 94 (4), 1246-1260.

18. Cornwallis, C.K. and Uller, T. (2009) Towards an evolutionary ecology of sexual traits. Trends. Ecol. Evol. 25 (3), 145-52.

19. Kokko, H. and Heubel, K. (2008) Condition-dependence, genotype-by-environment interactions and the lek paradox. Genetica 134 (1), 55-62.

20. Miller, C.W. and Svensson, E.I. (2014) Sexual selection in complex cnvironments. Annu. Rev. Entomol. 59 (1), 427-445.

21. Qvarnström, A. (2001) Context-dependent genetic benefits from mate choice. Trends Ecol. Evol. 16 (1), 5-7.

22. Dore, A.A. et al. (2018) The role of complex cues in social and reproductive plasticity. Behav. Ecol. Sociobiol. 72 (8).

23. Achorn, A.M. and Rosenthal, G.G. (2020) It's not about him: mismeasuring 'good genes' in sexual selection. Trends Ecol. Evol. 35 (3), 206-219.

24. Lewis, S. and South, A. (2012) The evolution of animal nuptial gifts. In Adv. Study of Behav., pp. 53-97, Elsevier.

25. Skulason, S. and Smith, T.B. (1995) Resource polymorphisms in vertebrates. Trends Ecol.Evol. 10 (9), 366-370.

26. Sih, A. et al. (2019) On the importance of individual differences in behavioural skill. Anim. Behav. 155, 307-317.

27. Pfennig, D.W. (1990) The adaptive significance of an environmentally-cued developmental switch in an anuran tadpole. Oecologia 85, 101-107.

28. Pfennig, K.S. (2000) Female spadefoot toads compromise on mate quality to ensure conspecific matings. Behav. Ecol. 11, 220-227.

29. Pfennig, K.S. (2008) Population differences in condition-dependent sexual selection may promote divergence in non-sexual traits. Evol. Ecol. Res. 10, 763-773.

30. Emlen, D.J. et al. (2012) A mechanism of extreme growth and reliable signaling in sexually selected ornaments and weapons. Science 337 (6096), 860-864.

31. Zahavi, A. and Zahavi, A. (1999) The handicap principle: A missing piece of Darwin's puzzle, Oxford University Press. 
32. Tomkins, J.L. et al. (2004) Genic capture and resolving the lek paradox. Trends Ecol. Evol. 19 (6), 323-328.

33. Proulx, S.R. (1999) Matings systems and the evolution of niche breadth. Am. Nat. 154 (1), 89-98.

34. Proulx, S.R. (2001) Female choice via indicator traits easily evolves in the face of recombination and migration. Evolution 55 (12), 2401-2411.

35. van Doorn, G.S. et al. (2009) On the origin of species by natural and sexual selection. Science 326 (5960), 1704-1707.

36. Levins, R. (1968) Evolution in changing environments, Princeton University Press.

37. Van Tienderen, P.H. (1991) Evolution of generalists and specialists in spatially heterogenous environments. Evolution 45, 1317-1331.

38. Moran, N.A. (1992) The evolutionary maintenance of alternative phenotypes. American Naturalist 139 (5), 971-989.

39. Berrigan, D. and Scheiner, S.M. (2004) Modeling the evolution of phenotypic plasticity. In Phenotypic plasticity: functional and conceptual approaches (DeWitt, T.J. and Scheiner, S.M. eds), pp. 82-97, Oxford University Press.

40. Whitman, D.W. and Agrawal, A.A. (2009) What is phenotypic plasticity and why is it important? In Phenotypic plasticity of insects (Whitman, D.W. and Ananthakrishnan, T.N. eds), pp. 1-63, Science Publishers.

41. Scheiner, S.M. (2020) The theory of the evolution of plasticity. In The theory of evolution (Scheiner, S.M. and Mindell, D.P. eds), pp. 254-272, University of Chicago Press.

42. Scheiner, S.M. (2002) Selection experiments and the study of phenotypic plasticity. $J$. Evol. Biol. 15, 889-898.

43. Schlichting, C.D. and Pigliucci, M. (1998) Phenotypic evolution: a reaction norm perspective, Sinauer Associates Incorporated.

44. Windig, J.J. et al. (2004) Genetics and mechanics of plasticity. In Phenotypic plasticity: functional and conceptual approaches (DeWitt, T.J. and Scheiner, S.M. eds), pp. 31-49, Oxford University Press.

45. Levis, N.A. and Pfennig, D.W. (2016) Evaluating 'plasticity-first' evolution in nature: key criteria and empirical approaches. Trends Ecol. Evol. 31 (7), 563-574.

46. Scheiner, S.M. et al. (2017) The genetics of phenotypic plasticity. XV. Genetic assimilation, the Baldwin effect, and evolutionary rescue. Ecol. Evol. 7, 8788-8803. 
47. Ghalambor, C.K. et al. (2007) Adaptive versus non-adaptive phenotypic plasticity and the potential for contemporary adaptation in new environments. Funct. Ecol. 21 (3), 394-407.

48. Murren, C.J. et al. (2015) Constraints on the evolution of phenotypic plasticity: limits and costs of phenotype and plasticity. Heredity 115 (4), 293-301.

49. Gavrilets, S. and Scheiner, S.M. (1993) The genetics of phenotypic plasticity. V. Evolution of reaction norm shape. J. Evol. Biol. 6 (1), 31-48.

50. Saltz, J.B. et al. (2018) Why does the magnitude of genotype-by-environment interaction vary? Ecol. Evol. 8 (12), 6342-6353.

51. Lafuente, E. and Beldade, P. (2019) Genomics of Developmental Plasticity in Animals. Front. Genet. 10 (720).

52. Fielenbach, N. and Antebi, A. (2008) C. elegans dauer formation and the molecular basis of plasticity. Genes Dev 22, 2149-2165.

53. Sommer, R.J. et al. (2017) The genetics of phenotypic plasticity in nematode feeding structures. Open Biology 7, 160332.

54. Weber, C. et al. (2020) Temperature-dependent sex determination is mediated by pSTAT3 repression of Kdm6b. Science 368 (6488), 303-306.

55. Bonduriansky, R. and Day, T. (2018) Extended heredity: a new understanding of inheritance and evolution, Princeton University Press.

56. Gilbert, S.F. and Epel, D. (2015) Ecological developmental biology: integrating epigenetics, medicine, and evolution, 2nd edn., Sinauer.

57. Levis, N.A. et al. (2017) Intraspecific adaptive radiation: Competition, ecological opportunity, and phenotypic diversification within species. Evolution 71 (10), 2496-2509.

58. Kelly, M. (2019) Adaptation to climate change through genetic accommodation and assimilation of plastic phenotypes. Phil. Trans. R. Soc. B 374 (1768).

59. Pfennig, D.W. et al. (2010) Phenotypic plasticity's impacts on diversification and speciation. Trends Ecol. Evol. 25 (8), 459-67.

60. Fox, R.J. et al. (2019) Beyond buying time: the role of plasticity in phenotypic adaptation to rapid environmental change. Phil. Trans. R. Soc. B 374 (1768).

61. Ashander, J. et al. (2016) Predicting evolutionary rescue via evolving plasticity in stochastic environments. Proc. R. Soc. B 283 (1839), 20161690. 
62. Kingsolver, J.G. and Diamond, S.E. (2011) Phenotypic selection in natural populations: what limits directional selection? Am. Nat. 177 (3), 346-357.

63. Kingsolver, J.G. and Pfennig, D.W. (2007) Patterns and power of phenotypic selection in nature. BioScience 57 (7), 561-572, 12.

64. Price, T.D. et al. (2003) The role of phenotypic plasticity in driving genetic evolution. Proc. R. Soc. B 270, 1433-1440.

65. Moczek, A.P. et al. (2011) The role of developmental plasticity in evolutionary innovation. Proc Biol Sci 278 (1719), 2705-13.

66. Levis, N.A. et al. (2018) Morphological novelty emerges from pre-existing phenotypic plasticity. Nat. Ecol.Evol. 2 (8), 1289.

67. Murren, C.J. et al. (2014) Evolutionary change in continuous reaction norms. Am. Nat. 183 (4), 453-467.

68. Levis, N.A. and Pfennig, D.W. (2019) Plasticity-led evolution: evaluating the key prediction of frequency-dependent adaptation. Proc. R. Soc. B 286 (1897), 20182754.

69. Nijhout, H.F. (2003) Development and evolution of adaptive polyphenisms. Evol. Dev. 5 (1), 9-18.

70. Ledon-Rettig, C.C. et al. (2014) Cryptic genetic variation in natural populations: a predictive framework. Integr. Comp. Biol. 54 (5), 783-93.

71. Maan, M.E. and Seehausen, O. (2011) Ecology, sexual selection and speciation. Ecol. Lett. 14 (6), 591-602.

72. Rundle, H.D. et al. (2006) The roles of natural and sexual selection during adaptation to a novel environment. Evolution 60 (11), 2218-2225.

73. Thibert-Plante, X. and Hendry, A.P. (2010) When can ecological speciation be detected with neutral loci? Mol. Ecol. 19 (11), 2301-14.

74. Wong, B.B.M. and Candolin, U. (2014) Behavioral responses to changing environments. Behav. Ecol. 26 (3), 665-673.

75. Moran, N.A. (1994) Adaptation and constraint in the complex life cycles of animals. Annu.Rev. Ecol. Syst. 25 (1), 573-600.

76. Wells, K.D. (2010) The ecology and behavior of amphibians, University of Chicago Press. 
77. Kasumovic, M.C.B., R.C. (2011) It's all who you know: the evolution of socially cued anticipatory plasticity as a mating strategy. Q. Rev. Biol. 86 (3), 181-197.

78. Snell-Rood, E. et al. (2015) Life history as a constraint on plasticity: developmental timing is correlated with phenotypic variation in birds. Heredity 115 (4), 379.

79. Xue, B. and Leibler, S. (2018) Benefits of phenotypic plasticity for population growth in varying environments. Proc. Natl. Acad. Sci. U.S.A. 115 (50), 12745-12750.

80. Snell-Rood, E.C. (2013) An overview of the evolutionary causes and consequences of behavioural plasticity. Anim. Behav. 85 (5), 1004-1011.

81. Travis, J. (2009) Phenotypic plasticity. In The Princeton guide to ecology (Levin, S.A. ed), pp. 65-71, Princeton University Press.

82. Pfennig, D.W. and Pfennig, K.S. (2012) Evolution's wedge: competition and the origins of diversity, University of California Press.

83. DeWitt, T.J. et al. (1998) Costs and limits of phenotypic plasticity. Trends in ecology \& evolution 13 (2), 77-81.

84. Dennis, S. et al. (2011) Phenotypic convergence along a gradient of predation risk. Proc. R. Soc. B 278 (1712), 1687-1696.

85. Winsor, G.L. and Innes, D.J. (2002) Sexual reproduction in Daphnia pulex (Crustacea: Cladocera): observations on male mating behaviour and avoidance of inbreeding. Freshw. Biol. 47 (3), 441-450.

86. Noach, E. et al. (1996) Phenotypic plasticity in morphological traits in two populations of Drosophila melanogaster. J. Evol. Biol. 9 (6), 831-844.

87. Byrne, P.G. and Rice, W.R. (2006) Evidence for adaptive male mate choice in the fruit fly Drosophila melanogaster. Proc. R. Soc. B 273 (1589), 917-922.

88. Blanckenhorn, W.U. (1998) Adaptive phenotypic plasticity in growth, development, and body size in the yellow dung fly. Evolution 52 (5), 1394-1407.

89. Ward, P.I. et al. (2002) Field experiments on the distributions of eggs of different phosphoglucomutase (PGM) genotypes in the yellow dung fly Scathophaga stercoraria (L.). Mol.Ecol. 11 (9), 1781-1785.

90. De Jong, M.A. et al. (2010) Geographic variation in thermal plasticity of life history and wing pattern in Bicyclus anynana. Clim. Res. 43 (1-2), 91-102.

91. San Martin, G. et al. (2011) Mate choice and sexual selection in a model butterfly species, Bicyclus anynana: state of the art. Proc. Neth. Entomol. Soc. Meet 22, 9-22. 
92. Hu, Y.et al. (2011) Development time and body size in Eupolyphaga sinensis along a latitudinal gradient from China. Environ. Entomol. 40 (1), 1-7.

93. Hu, Y. et al. (2010) Variation in sexual size dimorphism among populations: testing the differential-plasticity hypothesis. Entomol. Exp. Appl. 137 (2), 204-209.

94. Jiang, W. et al. (2013) Isolation and identification of two novel attractant compounds from Chinese cockroach (Eupolyphaga sinensis Walker) by combination of HSCCC, NMR and CD techniques. Molecules 18 (9), 11299-11310.

95. Grill, C.P.et al. (1997) The genetics of phenotypic plasticity in a colonizing population of the ladybird beetle, Hormonia axyridis. Heredity 78 (3), 261-269.

96. Bezzerides, A.L. et al. (2007) Elytra color as a signal of chemical defense in the Asian ladybird beetle Harmonia axyridis. Behav. Ecol. Sociobiol. 61 (9), 1401-1408.

97. Röder, G. et al. (2008) Counter-intuitive developmental plasticity induced by host quality. Proc. R. Soc. B 275 (1637), 879-885.

98. Labeyrie, E. et al. (2003) Mate choice and toxicity in two species of leaf beetles with different types of chemical defense. J. Chem. Ecol. 29 (7), 1665-1680.

99. Craig Stillwell, R. and Fox, C.W. (2009) Geographic variation in body size, sexual size dimorphism and fitness components of a seed beetle: local adaptation versus phenotypic plasticity. Oikos 118 (5), 703-712.

100. Savalli, U.M. and Fox, C.W. (1998) Sexual selection and the fitness consequences of male body size in the seed beetle Stator limbatus. Anim. Behav. 55 (2), 473-483.

101. Foray, V et al. (2011) Differential thermal performance curves in response to different habitats in the parasitoid Venturia canescens. Naturwissenschaften 98 (8), 683-691.

102. Chuine, A. et al. (2015) Consequences of genetic incompatibility on fitness and mate choice: the male point of view. Biol. J. Linn. Soc. 114 (2), 279-286.

103. Metzger, M. et al. (2010) Synergy in information use for mate finding: demonstration in a parasitoid wasp. Anim. Behav. 79 (6), 1307-1315.

104. Foo, S.A. et al. (2014) Increased temperature, but not acidification, enhances fertilization and development in a tropical urchin: potential for adaptation to a tropicalized eastern Australia. Evol. Appl. 7 (10), 1226-1237.

105. Sparks, K.M. et al. (2017) Paternal identity influences response of Acanthaster planci embryos to ocean acidification and warming. Coral Reefs 36 (1), 325-338. 
106. Kekäläinen, J. and Evans, J.P. (2018) Gamete-mediated mate choice: towards a more inclusive view of sexual selection. Proc. R. Soc. B 285 (1883), 20180836.

107. McCairns, R.S. and Bernatchez, L. (2010) Adaptive divergence between freshwater and marine sticklebacks: insights into the role of phenotypic plasticity from an integrated analysis of candidate gene expression. Evolution 64 (4), 1029-1047.

108. Bolnick, D.I. et al. (2015) Female stickleback prefer shallow males: sexual selection on nest microhabitat. Evolution 69 (6), 1643-1653.

109. Tinbergen, N. (1951) The study of instinct. Experientia 8, 3.

110. Robinson, B.W. and Wilson, D.S. (1996) Genetic variation and phenotypic plasticity in a trophically polymorphic population of pumpkinseed sunfish (Lepomis gibbosus). Evol. Ecol. 10 (6), 631-652.

111. Jarvis, W.M.C. et al. (2017) Flexible mate choice may contribute to ecotype assortative mating in pumpkinseed sunfish (Lepomis gibbosus). J. Evol. Biol. 30 (10), 1810-1820.

112. Rios-Cardenas, O. (2005) Patterns of parental investment and sexual selection in teleost fishes: do they support Bateman's principles? Integr. Comp. Biol. 45 (5), 885-894.

113. Crispo, E. and Chapman, L. (2010) Geographic variation in phenotypic plasticity in response to dissolved oxygen in an African cichlid fish. J. Evol. Biol. 23 (10), 2091-2103.

114. Gotanda, K. et al. (2011) Hypoxia and male behaviour in an African cichlid Pseudocrenilabrus multicolor victoriae. J. Fish Biol. 78 (7), 2085-2092.

115. Yamahira, K. et al. (2007) Inter-and intrapopulation variation in thermal reaction norms for growth rate: evolution of latitudinal compensation in ectotherms with a genetic constraint. Evolution 61 (7), 1577-1589.

116. Hamilton, J.B. et al. (1969) Competition for mating between ordinary and supermale Japanese medaka fish. Anim. Behav. 17, 168-176.

117. Gray, M.A. et al. (1999) Reproductive success and behavior of Japanese medaka (Oryzias latipes) exposed to 4-tert-octylphenol. Environ. Toxicol. Chem. 18 (11), 2587-2594.

118. McCairns, R.J.S. et al. (2016) The adaptive potential of subtropical rainbowfish in the face of climate change: heritability and heritable plasticity for the expression of candidate genes. Evol. Appl. 9 (4), 531-545.

119. Colléter, M. and Brown, C. (2011) Personality traits predict hierarchy rank in male rainbowfish social groups. Anim. Behav. 81 (6), 1231-1237. 
120. Young, M.J. et al. (2010) Pre- and post-mating sexual selection both favor large males in a rainbowfish. Behav. Ecol. Sociobiol. 64 (6), 915-925.

121. McCollum, S.A. and Van Buskirk, J. (1996) Costs and benefits of a predator-induced polyphenism in the gray treefrog Hyla chrysoscelis. Evolution 50 (2), 583-593.

122. Semlitsch, R.D. and Gibbons, J.W. (1988) Fish predation in size-structured populations of treefrog tadpoles. Oecologia 75 (3), 321-326.

123. Van Buskirk, J. and McCollum, S.A. (1999) Plasticity and selection explain variation in tadpole phenotype between ponds with different predator composition. Oikos, 31-39.

124. Gerhardt, H.C. (1991) Female mate choice in treefrogs: static and dynamic acoustic criteria. Anim. Behav. 42 (4), 615-635.

125. Gerhardt, H.C. (1994) Reproductive character displacement of female mate choice in the grey treefrog, Hyla chrysoscelis. Anim. Behav. 47 (4), 959-969.

126. Relyea, R.A. (2002) Costs of phenotypic plasticity. Am. Nat. 159 (3), 272-282.

127. Berven, K.A. (1981) Mate choice in the wood frog, Rana sylvatica. Evolution 35 (4), 707-722.

128. Sänen, K.R. et al. (2003) Geographic variation in acid stress tolerance of the moor frog, Rana arvalis. I. Local adaptation. Evolution 57 (2), 352-362.

129. Sherman, C.D.H. et al. (2010) Female Choice for Males with Greater Fertilization Success in the Swedish Moor Frog, Rana arvalis. PLOS ONE 5 (10), e13634.

130. Jockusch, E.L. (1997) Geographic variation and phenotypic plasticity of number of trunk vertebrae in slender salamanders, Batrachoseps (Caudata: Plethodontidae). Evolution 51 (6), 1966-1982.

131. Wake, D.B. (2006) Problems with species: patterns and processes of species formation in salamanders 1. Ann. Missouri Bot. Gard. 93 (1), 8-23.

132. Meyers, L.A. and Bull, J.J. (2002) Fighting change with change: adaptative variation in an uncertain world. Trends Ecol Evol 17 (12), 551-557.

133. Nijhout, H.F. (2003) Development and evolution of adaptive polyphenisms. Evolution and Development 5 (1), 9-18.

134. Ghalambor, C.K. et al. (2015) Non-adaptive plasticity potentiates rapid adaptive evolution of gene expression in nature. Nature 525 (7569), 372-375. 
135. Via, S. et al. (1995) Adaptive phenotypic plasticity: consensus and controversy. Trends in Ecology \& Evolution 10 (5), 212-217.

136. DeWitt, T.J. and Langerhans, R.B. (2004) Integrated solutions to environmental heterogeneity. In Phenotypic plasticity: functional and conceptual approaches (DeWitt, T.J. and Scheiner, S.M. eds), pp. 98-111, Oxford University Press.

137. Schlichting, C.D. (2004) The role of phenotypic plasticity in diversification. In Phenotypic plasticity: functional and conceptual approaches (DeWitt, T.J. and Scheiner, S.M. eds), pp. 191-200, Oxford University Press.

138. Huey, R.B.et al. (2003) Behavioral drive versus behavioral inertia in evolution: a null model approach. American Naturalist 161, 357-366.

139. Agrawal, A.A. (2001) Phenotypic plasticity in the interactions and evolution of species. Science (Washington, D. C.) 294, 321-326.

140. Aubret, F. and Shine, R. (2009) Genetic assimilation and the postcolonization erosion of phenotypic plasticity in island Tiger Snakes. Current Biology 19 (22), 1932-1936.

141. Badyaev, A.V. (2005) Stress-induced variation in evolution: from behavioural plasticity to genetic assimilation. Proceedings of the Royal Society B: Biological Sciences 272, 877886.

142. Lande, R. (2009) Adaptation to an extraordinary environment by evolution of phenotypic plasticity and genetic assimilation. Journal of Evolutionary Biology 22 (7), 1435 1446.

143. Levis, N.A. and Pfennig, D.W. (2016) Evaluating 'plasticity-first' evolution in nature: key criteria and empirical approaches. Trends In Ecology \& Evolution 31 (7), 563-574.

144. Moczek, A.P. et al. (2011) The role of developmental plasticity in evolutionary innovation. Proceedings of the Royal Society B: Biological Sciences 278, 2705-2713.

145. Pfennig, D.W. et al. (2010) Phenotypic plasticity's impacts on diversification and speciation. Trends in Ecology and Evolution 25, 459-467.

146. Pigliucci, M. et al. (2006) Phenotypic plasticity and evolution by genetic assimilation. Journal of Experimental Biology 209, 2362-2367.

147. Price, T.D. et al. (2003) The role of phenotypic plasticity in driving genetic evolution. Proceedings of the Royal Society B: Biological Sciences 270, 1433-1440.

148. Schneider, R.F. and Meyer, A. (2017) How plasticity, genetic assimilation and cryptic genetic variation may contribute to adaptive radiations. Molecular Ecology 26 (1), 330-350. 
149. West-Eberhard, M.J. (1989) Phenotypic plasticity and the origins of diversity. Annual Review of Ecology and Systematics 20, 249-278.

150. Wund, M.A. (2012) Assessing the impacts of phenotypic plasticity on evolution. Integrative and Comparative Biology 52 (1), 5-15.

151. Scheiner, S.M. (1993) Genetics and evolution of phenotypic plasticity. Annual Review of Ecology and Systematics 24, 35-68.

152. Scheiner, S.M. (2002) Selection experiments and the study of phenotypic plasticity. Journal of Evolutionary Biology 15, 889-898.

153. Sultan, S.E. (1995) Phenotypic plasticity and plant adaptation. Acta Botanica Neerlandica 44, 363-383.

154. Alpert, P. and Simms, E.L. (2002) The relative advantages of plasticity and fixity in different environments: When is it good for a plant to adjust? Evolutionary Ecology 16, 285297.

155. Lind, M.I. et al. (2010) Gene flow and selection on phenotypic plasticity in an island system of Rana temporaria. Evolution 65, 684-697.

156. Darwin, C. (1871) The descent of man, and selection in relation to sex, John Murray.

157. Wong, B.B.M. and Candolin, U. (2015) Behavioral responses to changing environments. Behavioral Ecology 26 (3), 665-673.

158. Servedio, M.R. and Boughman, J.W. (2017) The role of sexual selection in local adaptation and speciation. Annual Review of Ecology, Evolution, and Systematics 48, 85109.

159. Candolin, U. and Heuschele, J. (2008) Is sexual selection beneficial during adaptation to environmental change? Trends in Ecology \& Evolution 23 (8), 446-452.

160. Thibert-Plante, X. and Gavrilets, S. (2013) Evolution of mate choice and the so-called magic traits in ecological speciation. Ecol Lett 16 (8), 1004-13.

161. Maan, M.E. and Seehausen, O. (2011) Ecology, sexual selection and speciation. Ecology Letters.

162. Kokko, H. and Brooks, R. (2003) Sexy to die for? Sexual selection and the risk of extinction. Annales Zoologici Fennici 40, 207-219.

163. Fernandes Martins, M.J. et al. (2018) High male sexual investment as a driver of extinction in fossil ostracods. Nature 556, 366-369. 
164. Jennions, M.D. and Petrie, M. (1997) Variation in mate choice and mating preferences: A review of causes and consequences. Biological Reviews Of The Cambridge Philosophical Society 72 (2), 283-327.

165. Cotton, S. et al. (2006) Sexual selection and condition-dependent mate preferences. Current Biology 16 (17), R755-R765.

166. Pfennig, K.S. (2007) Facultative mate choice drives adaptive hybridization. Science 318, 965-967.

167. Pfennig, K.S. (2000) Female spadefoot toads compromise on mate quality to ensure conspecific matings. Behavioral Ecology 11, 220-227.

168. Pfennig, K.S. (2008) Population differences in condition-dependent sexual selection may promote divergence in non-sexual traits. Evolutionary Ecology Research 10, 763-773.

169. Pfennig, K.S. and Rice, A.M. (2014) Reinforcement generates reproductive isolation between neighbouring populations of spadefoot toads. Proceedings of the Royal Society B: Biological Sciences 281, 20140949.

170. Pfennig, D.W. (1992) Proximate and functional causes of polyphenism in an anuran tadpole. Functional Ecology 6, 167-174.

171. Bragg, A.N. (1965) Gnomes of the night: the spadefoot toads, University of Pennsylvania Press.

172. Pfennig, K.S. and Pfennig, D.W. (2005) Character displacement as the 'best of a bad situation': fitness trade-offs resulting from selection to minimize resource and mate competition. Evolution 59, 2200-2208.

173. Paull, J.S. et al. (2012) Increased competition as a cost of specialization during the evolution of resource polymorphism. Biological Journal of the Linnean Society 107, 845853.

174. Levis, N.A. et al. (2015) An inducible offense: carnivore morph tadpoles induced by tadpole carnivory. Ecology and Evolution 5 (7), 1405-1411.

175. Martin, R.A. and Pfennig, D.W. (2011) Evaluating the targets of selection during character displacement. Evolution 65, 2946-2958.

176. Pfennig, D.W. (1999) Cannibalistic tadpoles that pose the greatest threat to kin are most likely to discriminate kin. Proceedings of the Royal Society of London: Biological Sciences 266, 57-81.

177. Pfennig, K.S. et al. (2015) Sexual selection's impacts on ecological specialization: an experimental test. Proceedings of the Royal Society B: Biological Sciences 282 (1807). 
178. Martin, R.A. and Pfennig, D.W. (2010) Field and experimental evidence that competition and ecological opportunity promote resource polymorphism. Biological Journal of the Linnean Society 100, 73-88.

179. Martin, R.A. and Pfennig, D.W. (2012) Widespread disruptive selection in the wild is associated with intense resource competition. BMC Evolutionary Biology 12, 136.

180. Martin, R.A. and Pfennig, D.W. (2009) Disruptive selection in natural populations: the roles of ecological specialization and resource competition. American Naturalist 174, 268281.

181. Pfennig, D.W. et al. (2007) Field and experimental evidence for competition's role in phenotypic divergence. Evolution 61, 257-271.

182. Frankino, W.A. and Pfennig, D.W. (2001) Condition-dependent expression of trophic polyphenism: effects of individual size and competitive ability. Evolutionary Ecology Research 3, 939-951.

183. Pfennig, D.W. et al. (2006) Ecological opportunity and phenotypic plasticity interact to promote character displacement and species coexistence. Ecology 87 (3), 769-779.

184. Gosner, K.L. (1960) A simplified table for staging anuran embryos with notes on identification. Herpetologica 16, 183-190.

185. Martin, R.A. and Pfennig, D.W. (2010) Maternal investment influences expression of resource polymorphism in amphibians: implications for the evolution of novel resource-use phenotypes. PLoS One 5, e9117.

186. Pfennig, D.W. and Martin, R.A. (2009) A maternal effect mediates rapid population divergence and character displacement in spadefoot toads. Evolution 63, 898-909.

187. Levis, N.A. et al. (2017) Genetic accommodation in the wild: evolution of gene expression plasticity during character displacement. Journal of Evolutionary Biology 30 (9), 1712-1723.

188. Levis, N.A. and Pfennig, D.W. (2018) A test of alternative hypotheses for how canalizing selection and nongenetic inheritance combine to shape the evolution of phenotypic plasticity. Evolution in review.

189. Waddington, C.H. (1953) Genetic assimilation of an acquired character. Evolution 7, 118-126.

190. Ehrenreich, I.M. and Pfennig, D.W. (2016) Genetic assimilation: a review of its potential proximate causes and evolutionary consequences. Annals of Botany 117, 769-779. 
191. Scheiner, S.M. et al. (2017) The genetics of phenotypic plasticity. XV. Genetic assimilation, the Baldwin effect, and evolutionary rescue. Ecology and Evolution 7, 87888803.

192. Murren, C.J. et al. (2015) Constraints on the evolution of phenotypic plasticity: limits and costs of phenotype and plasticity. Heredity 115, 293-301.

193. Boughman, J.W. (2007) Condition-dependent expression of red colour differs between stickleback species. Journal of Evolutionary Biology 20 (4), 1577-1590.

194. Brock, C.D. et al. (2017) Phenotypic plasticity drives a depth gradient in male conspicuousness in threespine stickleback, Gasterosteus aculeatus. Evolution 71, 2022-2036.

195. Lorch, P.D. et al. (2003) Condition-dependent sexual selection can accelerate adaptation. Evolutionary Ecology Research 5 (6), 867-881.

196. Long, T.A.F. et al. (2012) The effect of sexual selection on offspring fitness depends on the nature of genetic variation. Current Biology 22 (3), 204-208.

197. Gross, Mart R. (1996) Alternative reproductive strategies and tactics: Diversity within sexes. Trends in Ecology \& Evolution 11 (2), 92-98.

198. Moczek, A.P. and Emlen, D.J. (2000) Male horn dimorphism in the scarab beetle Onthophagus taurus: do alternative reproductive tactics favor alternative phenotypes? Animal Behaviour 59, 459-466.

199. Kraft, P.G. et al. (2005) Predator-mediated phenotypic plasticity in tadpoles of the striped marsh frog, Limnodynastes peronii. Austral Ecol 30 (5), 558-563.

200. LaFiandra, E.M. and Babbitt, K.J. (2004) Predator induced phenotypic plasticity in the pinewoods tree frog, Hyla femoralis: necessary cues and the cost of development. Oecologia 138 (3), 350-359.

201. Michimae, H. and Hangui, J.-I. (2008) A trade-off between prey-and predator-induced polyphenisms in larvae of the salamander Hynobius retardatus. Behav. Ecol. Sociobiol. 62 (5), 699-704.

202. Relyea, R.A. (2002) Competitor-induced plasticity in tadpoles: consequences, cues, and connections to predator-induced plasticity. Ecol. Monogr. 72 (4), 523-540.

203. Smith, D.C. and Van Buskirk, J. (1995) Phenotypic design, plasticity, and ecological performance in two tadpole species. Am Nat 145 (2), 211-233.

204. Touchon, J.C. and Warkentin, K.M. (2008) Fish and dragonfly nymph predators induce opposite shifts in color and morphology of tadpoles. Oikos 117 (4), 634-640. 
205. Van Buskirk, J. and Schmidt, B.R. (2000) Predator-induced phenotypic plasticity in larval newts: trade-offs, selection, and variation in nature. Ecology 81 (11), 3009-3028.

206. Kraft, P. et al. (2006) Predator-induced phenotypic plasticity in tadpoles: extension or innovation? J. Evol. Biol. 19 (2), 450-458.

207. McIntyre, P.B. et al. (2004) Effects of behavioral and morphological plasticity on risk of predation in a Neotropical tadpole. Oecologia 141 (1), 130-138.

208. Van Buskirk, J. et al. (1997) Natural selection for environmentally induced phenotypes in tadpoles. Evolution 51 (6), 1983-1992.

209. Relyea, R.A. (2004) Fine-tuned phenotypes: tadpole plasticity under 16 combinations of predators and competitors. Ecology 85 (1), 172-179.

210. Dodd, C.K. (2013) Frogs of the United States and Canada, 2-vol. set, JHU Press.

211. Martin, R.A. and Pfennig, D.W. (2009) Disruptive selection in natural populations: the roles of ecological specialization and resource competition. Am Nat 174 (2), 268-81.

212. Martin, R.A. and Pfennig, D.W. (2010) Field and experimental evidence that competition and ecological opportunity promote resource polymorphism. Biol. J. Linn. Soc. $100,73-88$.

213. Martin, R.A. and Pfennig, D.W. (2012) Widespread disruptive selection in the wild is associated with intense resource competition. BMC Evol Biol 12, 136.

214. Pfennig, D.W. et al. (1993) Kin recognition and cannibalism in spadefoot toad tadpoles. Animal Behaviour 46 (1), 87-94.

215. Pomeroy, L.V. (1981) Developmental polymorphism in the tadpoles of the spadefoot toad Scaphiopus multiplicatus. PhD diss. University of California, Riverside, USA.

216. Schoeppner, N.M. and Relyea, R.A. (2005) Damage, digestion, and defence: the roles of alarm cues and kairomones for inducing prey defences. Ecol. Lett. 8 (5), 505-512.

217. Lee, N. et al. (2017) Frogs exploit statistical regularities in noisy acoustic scenes to solve cocktail-party-like problems. Curr Biol 27 (5), 743-750.

218. Ward, J.L. et al. (2013) Multitasking males and multiplicative females: dynamic signalling and receiver preferences in Cope's grey treefrog. Anim. Behav. 86 (2), 231-243.

219. Team, R.C., R: A language and environment for statistical computing, R Foundation for Statistical Computing, Vienna, Austria, 2019. 
220. Kuznetsova, A. et al. (2017) lmerTest Package: Tests in Linear Mixed Effects Models. $J$ Stat Softw 82 (13), 26.

221. Hedges, L.V. and Olkin, I. (2014) Statistical methods for meta-analysis, Academic press.

222. Peig, J. and Green, A.J. (2009) New perspectives for estimating body condition from mass/length data: the scaled mass index as an alternative method. Oikos 118 (12), 1883-1891.

223. Touchon, J.C. and Robertson, J.M. (2018) You cannot have it all: Heritability and constraints of predator-induced developmental plasticity in a Neotropical treefrog. Evolution 72 (12), 2758-2772.

224. Moczek, A.P. (2007) Developmental capacitance, genetic accommodation, and adaptive evolution. Evolution \& development 9 (3), 299-305.

225. Levis, N.A. and Pfennig, D.W. (2020) Plasticity-led evolution: A survey of developmental mechanisms and empirical tests. Evolution \& Development 22 (1-2), 71-87.

226. Bui, L.T. and Ragsdale, E.J. (2019) Multiple plasticity regulators reveal targets specifying an induced predatory form in nematodes. Molecular Biology and Evolution 36 (11), 2387-2399.

227. Levis, N.A. et al. (2021) Transcriptomic bases of a polyphenism. J Exp Zool B Mol Dev Evol 336 (6), 482-495.

228. Ragsdale, E.J. et al. (2013) A developmental switch coupled to the evolution of plasticity acts through a sulfatase. Cell 155 (4), 922-933.

229. Ah-King, M. and Gowaty, P.A. (2016) A conceptual review of mate choice: stochastic demography, within-sex phenotypic plasticity, and individual flexibility. Ecol Evol 6 (14), 4607-42.

230. Kelly, P.W. et al. (2021) Adaptive Plasticity as a Fitness Benefit of Mate Choice. Trends in Ecology \& Evolution.

231. Warren, I.A. et al. (2013) A general mechanism for conditional expression of exaggerated sexually-selected traits. Bioessays 35 (10), 889-99.

232. Zinna, R. et al. (2018) Sexual dimorphism and heightened conditional expression in a sexually selected weapon in the Asian rhinoceros beetle. Molecular Ecology 27 (24), 50495072 .

233. Nielsen, M.E. and Papaj, D.R. (2017) Why Have Multiple Plastic Responses? Interactions between Color Change and Heat Avoidance Behavior in Battus philenor Larvae. The American Naturalist 189 (6), 657-666. 
234. (2021) Phenotypic Plasticity \& Evolution: Causes, Consequences, Controversies, 1st edn., CRC Press.

235. Welch, A.M. (2003) GENETIC BENEFITS OF A FEMALE MATING PREFERENCE IN GRAY TREE FROGS ARE CONTEXT-DEPENDENT. 57 (4), 883-893.

236. Welch, A.M. et al. (1998) Call Duration as an Indicator of Genetic Quality in Male Gray Tree Frogs. Science 280 (5371), 1928-1930.

237. Achorn, A.M. and Rosenthal, G.G. (2019) It's not about him: mismeasuring 'good genes' in sexual selection. Trends Ecol. Evol.

238. Bradshaw, A.D. (1965) Evolutionary significance of phenotypic plasticity in plants. Advances in genetics 13, 115-155.

239. Forsman, A.J.H. (2015) Rethinking phenotypic plasticity and its consequences for individuals, populations and species. $115(4), 276$.

240. Windig, J.J. et al. (2004) Genetics and mechanics of plasticity. Phenotypic plasticity, 31-49.

241. Andersson, M. (2006) Condition-dependent indicators in sexual selection: development of theory and tests. Essays in Animal Behaviour, Celebrating 50 Years of Animal Behaviour, 253-267.

242. Prokop, Z.M. et al. (2012) META-ANALYSIS SUGGESTS CHOOSY FEMALES GET SEXY SONS MORE THAN “GOOD GENES”. 66 (9), 2665-2673.

243. Hill, G.E. (2015) Sexiness, Individual Condition, and Species Identity: The Information Signaled by Ornaments and Assessed by Choosing Females. Evolutionary Biology 42 (3), 251-259.

244. Kokko, H. et al. (2006) Unifying and Testing Models of Sexual Selection. Annual Review of Ecology, Evolution, and Systematics 37 (1), 43-66.

245. Ryan, M.J. and Cummings, M.E. (2013) Perceptual Biases and Mate Choice. 44 (1), 437-459.

246. Levis, N.A. and Pfennig, D.W. (2019) How stabilizing selection and nongenetic inheritance combine to shape the evolution of phenotypic plasticity. Journal of evolutionary biology.

247. Ledón-Rettig, C.C. and Ragsdale, E.J. (2021) Physiological Mechanisms and the Evolution of Plasticity. In Phenotypic Plasticity \& Evolution, pp. 113-137. 